\title{
Novel N-substituted aminobenzamide scaffold derivatives targeting the dipeptidyl peptidase-IV enzyme
}

This article was published in the following Dove Press journal:

Drug Design, Development and Therapy

16 January 2014

Number of times this article has been viewed

\author{
Qosay A Al-Balas' \\ Munia F Sowaileh' \\ Mohammad A Hassan' \\ Amjad M Qandil ${ }^{1,2}$ \\ Karem H Alzoubi ${ }^{3}$ \\ Nizar M Mhaidat ${ }^{3}$ \\ Ammar M Almaaytah ${ }^{4}$ \\ Omar F Khabour ${ }^{5}$ \\ 'Department of Medicinal Chemistry \\ and Pharmacognosy, Faculty of \\ Pharmacy, Jordan University of \\ Science and Technology, Irbid, \\ Jordan; ${ }^{2}$ Pharmaceutical Sciences \\ Department, College of Pharmacy, \\ King Saud bin Abdulaziz University \\ for Health Sciences, Riyadh, Saudi \\ Arabia; ${ }^{3}$ Department of Clinical \\ Pharmacy, Faculty of Pharmacy, \\ Jordan University of Science and \\ Technology, Irbid, Jordan; ${ }^{4}$ Department \\ of Pharmaceutical Technology, Faculty \\ of Pharmacy, Jordan University of \\ Science and Technology, Irbid, Jordan; \\ ${ }^{5}$ Department of Medical Laboratory \\ Sciences, Faculty of Applied Medical \\ Sciences, Jordan University of Science \\ and Technology, Irbid, Jordan
}

Background: The dipeptidyl peptidase-IV (DPP-IV) enzyme is considered a pivotal target for controlling normal blood sugar levels in the body. Incretins secreted in response to ingestion of meals enhance insulin release to the blood, and DPP-IV inactivates these incretins within a short period and stops their action. Inhibition of this enzyme escalates the action of incretins and induces more insulin to achieve better glucose control in diabetic patients. Thus, inhibition of this enzyme will lead to better control of blood sugar levels.

Methods: In this study, computer-aided drug design was used to help establish a novel $\mathrm{N}$-substituted aminobenzamide scaffold as a potential inhibitor of DPP-IV. CDOCKER software available from Discovery Studio 3.5 was used to evaluate a series of designed compounds and assess their mode of binding to the active site of the DPP-IV enzyme. The designed compounds were synthesized and tested against a DPP-IV enzyme kit provided by Enzo Life Sciences. The synthesized compounds were characterized using proton and carbon nuclear magnetic resonance, mass spectrometry, infrared spectroscopy, and determination of melting point.

Results: Sixty-nine novel compounds having an N-aminobenzamide scaffold were prepared, with full characterization. Ten of these compounds showed more in vitro activity against DPP-IV than the reference compounds, with the most active compounds scoring $38 \%$ activity at $100 \mu \mathrm{M}$ concentration.

Conclusion: The N-aminobenzamide scaffold was shown in this study to be a valid scaffold for inhibiting the DPP-IV enzyme. Continuing work could unravel more active compounds possessing the same scaffold.

Keywords: diabetes mellitus, dipeptidyl peptidase-IV, aminobenzamide derivatives, hypoglycemic activity, CDOCKER software

\section{Introduction}

Diabetes is a group of metabolic disorders characterized by hyperglycemia caused by insulin deficiency (type 1 diabetes) or insulin resistance (type 2 diabetes). ${ }^{1}$ The classical symptoms of diabetes are polyuria, polydipsia, and polyphagia, with other symptoms including weight loss, fatigue, frequent infections, blurred vision, slower healing of injuries, and numbness in the extremities. ${ }^{2}$ The impact of diabetes on patients' lives and on society's economy elicits a compelling need to deal with diabetes more seriously. ${ }^{3}$ The main focus of treatment for diabetes is to maintain blood glucose levels in the normal range throughout the day. Tight control of glucose levels is essential in slowing progression of the disease and in preventing its complications. ${ }^{4}$

A valid target when designing antidiabetic agents is inhibition of the dipeptidyl peptidase-IV enzyme (DPP-IV). DPP-IV was discovered in 1966, but its role in glucose
Correspondence: Qosay A Al-Balas;

Munia F Sowaileh

Department of Medicinal Chemistry

and Pharmacognosy, Faculty of

Pharmacy, Jordan University of

Science and Technology,

PO Box 3030, Irbid 221 I0, Jordan

Tel +962776337216

Fax+962 2720I 075

Email qabalas@just.edu.jo; monia@live.com 
homeostasis took some time to become clear, ${ }^{5}$ and it was not until 1998 that inhibition of DPP-IV was suggested as a possible route to manage diabetes. ${ }^{6}$ Since then, a number of DPP-IV inhibitors have appeared in the literature. DPP-IV is an example of great demand making a novel target a perfect investment for pharmaceutical companies, and this has resulted in four drugs being approved by the US Food and Drug Administration and six drugs being marketed world-

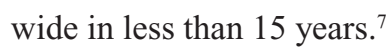

DPP-IV is composed of a single polypeptide chain containing 766 amino acids. It can be roughly divided into three regions, ie, a catalytic region, a cysteine-rich region, and a highly glycosylated region, in addition to a transmembrane domain at the N-terminus in the case of membrane-bound DPP-IV. DPP-IV monomers join to form homodimers. ${ }^{8,9}$

DPP-IV is a serine-type peptidase, meaning that its catalytic activity is essentially based on an interaction with the amino acid serine at its active site. ${ }^{10}$ The catalytic domain of DPP-IV consists of Ser630, Asp708, and His740. ${ }^{11}$ Although distant from the Ser-Asp-His catalytic triad, Glu205 and Glu206 play a critical role in the activity of this enzyme. ${ }^{12}$

An important feature of the active site of DPP-IV is the presence of a tight hydrophobic pocket, known as the $\mathrm{S} 1$ pocket (see Figure 1). This pocket is responsible for the specificity of the DPP-IV substrate for proline and its tolerance for alanine and glycine. ${ }^{13}$

The rationale for this work is based on a previous investigation performed in our laboratory where screening using a DPP-IV enzyme assay kit indicated that compound 1 shown in Figure 2 has a $28 \%$ inhibitory effect on DPP-IV; this structure has subsequently served as a guide compound for the design of a series of DPP-IV inhibitors. The present work describes the synthesis of 69 novel N-substituted aminobenzamide derivatives that were fully characterized with ${ }^{1} \mathrm{H}$ nuclear magnetic resonance (NMR), ${ }^{13} \mathrm{C}$ NMR, and mass and infrared spectroscopy, along with evaluation of their DPP-IV inhibition activity. Using computer modeling techniques to introduce modifications that we assumed would improve binding, a series of aminobenzamides was investigated.

\section{Materials and methods In silico docking}

A search for the DPP-IV enzyme crystal structure in the Protein Data Bank ${ }^{14}$ was conducted on November 30, 2011 using the search terms "DPP-IV" and "dipeptidyl peptidaseIV". The hits were limited to enzymes extracted from Homo sapiens only, and each hit was inspected to check the protein, ligand, and resolution. In the "Prepare Protein" protocol, the default parameter values were mostly kept the same. The proposed compounds were prepared using the "Prepare Ligand" protocol, also with default parameters.

Docking was accomplished using the CDOCKER protocol available from Accelrys (San Diego, CA, USA). ${ }^{15}$ The following parameters were used: Top Hits, 10; Random Conformations, 10; Orientations to Refine, 10; Forcefield, CHARMM; and Use Full Potential, False. Careful inspection of all crystal structures showed that the following amino acids were involved in binding the substrates (amino acids are arranged according to the frequency of bond formation in descending order): H-bond-forming amino acids, ie, Glu206, Tyr662, Glu205, Arg125, Asn710, Tyr547, Arg358, Tyr631, Ser209, Tyr585, Val207, and Tyr666; and Pi-Pi interactionforming amino acids, ie, His740, Tyr662, Phe337, Tyr457, Arg125, Arg358, and Trp659.

\section{Chemistry}

Bulk solvents and chemicals were purchased through local vendors without any further purification or distillation. The progress of reactions was qualitatively analyzed by thin layer chromatography performed on Merck aluminum thin layer chromatography plates (DC Kieselgel 60 F254). An ultraviolet lamp was used to visualize the thin layer chromatography plate. New compounds were characterized by ${ }^{1} \mathrm{H}-\mathrm{NMR}$ and ${ }^{13} \mathrm{C}$-NMR using a $400 \mathrm{mHz}$ Avance Ultrashield spectrometer (Bruker, Ettlingen, Germany); the spectra were obtained in parts per million using automatic calibration to the residual proton peak of the solvent, ie, dimethylsulfoxide (DMSO)-d6. The infrared spectra of the synthesized compounds were recorded on a Thermo-Nicolet Avatar 360 FT-IR, (Thermo Fisher Scientific, Rockville, MD, USA). A liquid chromatography-tandem mass spectrometry system (API 3200, Applied Biosystems-MDS SCIEX, Foster City, CA, USA), employing positive mode, was used to characterize the compounds, with the electrospray ionization source operated at $5.0-5.5 \mathrm{kV}$, the capillary heater at $350^{\circ} \mathrm{C}$, and a sheath gas pressure of 45 psi. The enzyme activity plates were read using a universal microplate reader (ELx800, Bio-Tek Instruments Inc, Winooski, VT, USA).

\section{Synthesis of anilinoketones from substituted $\alpha$-bromoketones $(52-84,87,88)$}

Amino-N-substituted phenylbenzamide (one equivalent), potassium carbonate (one equivalent), and $\alpha$-bromoketone (one equivalent) were dissolved in dimethylformamide $(20 \mathrm{~mL})$. The reaction mixture was stirred for 8 hours. The reaction contents 
A

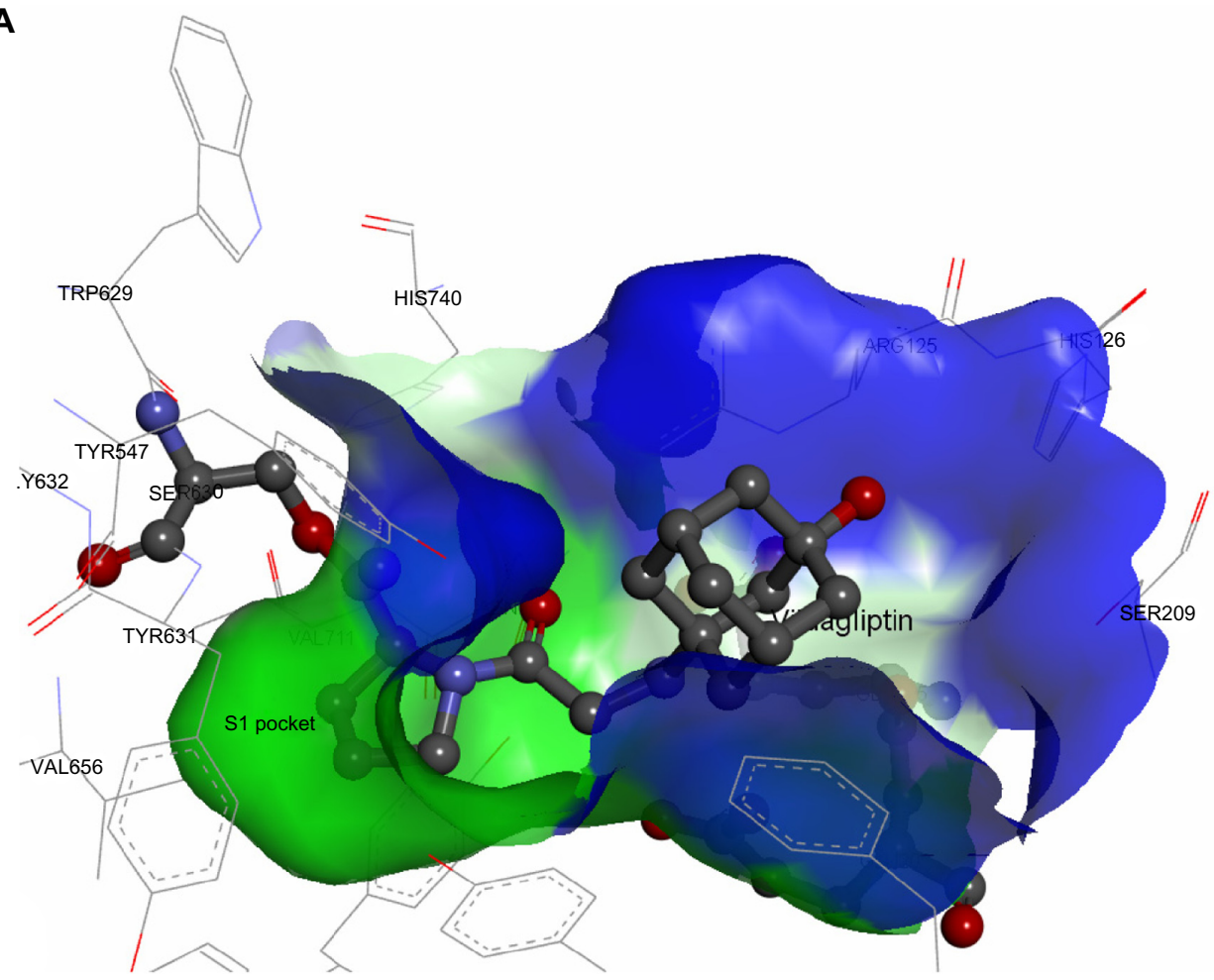

B

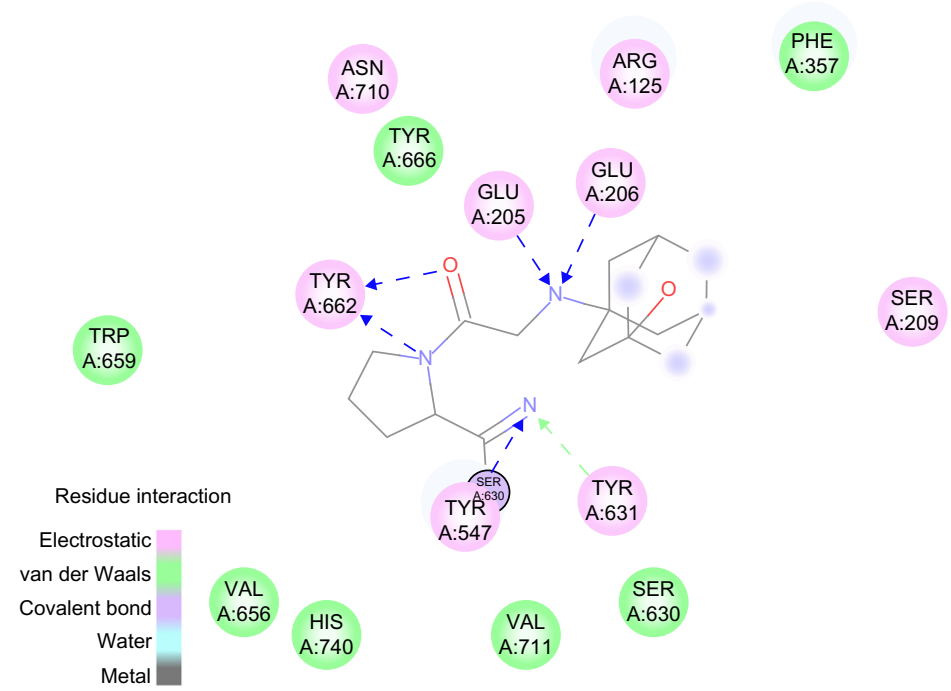

Figure I (A) Three-dimensional X-ray structure of the binding pocket of DPP-IV with an inhibitor (vildagliptin). (B) Two-dimensional diagram of vildagliptin binding DPP-IV active site showing different types of interactions.

Abbreviation: DPP-IV, dipeptidyl peptidase-V.

were then poured over water with stirring, and the mixture was left until the product precipitated. The product was then filtered, dried, and recrystallized from ethanol.

\section{4-(2-oxo-2-phenylethylamino)-}

\section{$\mathrm{N}$-phenylbenzamide (52)}

Prepared by reacting $23(1.06 \mathrm{~g}, 5 \mathrm{mmol})$ and $\mathrm{H1}(1 \mathrm{~g}, 5 \mathrm{mmol})$ in the presence of potassium carbonate $(0.5 \mathrm{~g}, 5 \mathrm{mmol})$, to yield $0.6 \mathrm{~g}(36 \%)$ of 52 as light yellow crystals. Melting point $186^{\circ} \mathrm{C}-188^{\circ} \mathrm{C}$; IR $3,375,3,294,3,039,1,685,1,641$, $1,319 \mathrm{~cm}^{-1} ;{ }^{1} \mathrm{H}$ NMR (400 mHz, DMSO-d6): $\delta 4.79$ (d, 2H, $\mathrm{CH} 2, \mathrm{~J}=5.4 \mathrm{~Hz}$ ), 6.53 (t, 1H, NH-Ar, J =5.3 Hz), 6.75 (d, $2 \mathrm{H}, \mathrm{Ar}-\mathrm{H}, \mathrm{J}=8.5 \mathrm{~Hz}$ ), 7.04 (t, 1H, Ar-H, J =7.2 Hz), 7.31 (t, $2 \mathrm{H}, \mathrm{Ar}-\mathrm{H}, \mathrm{J}=7.8 \mathrm{~Hz}$ ), 7.58 (t, 2H, Ar-H, J =7.6 Hz), 7.68 (d, $1 \mathrm{H}, \mathrm{Ar}-\mathrm{H}, \mathrm{J}=7.3 \mathrm{~Hz}), 7.74(\mathrm{~d}, 2 \mathrm{H}, \mathrm{Ar}-\mathrm{H}, \mathrm{J}=8.2 \mathrm{~Hz}), 7.77$ (d, 2H, Ar-H, J = 8.4 Hz), 8.09 (d, 2H, Ar-H, J = 8 Hz) 10.23 


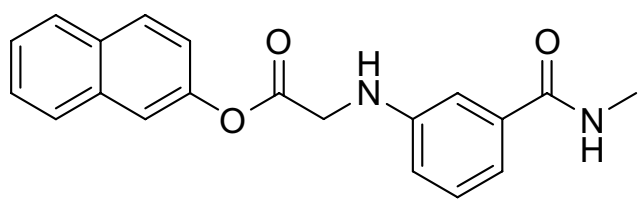

Figure 2 Structure of compound I, as the starting scaffold.

(s, ${ }^{1} \mathrm{H}, \mathrm{CO}-\mathrm{NH}$ ); ${ }^{13} \mathrm{C}-\mathrm{NMR}$ (DMSO-d6): $\delta 52.13,114.12$, $122.85,124.37,125.65,130.56,131.12,131.47,131.83$, 136.30, 137.64, 142.32, 153.79, 167.98, 198.85; MS (EI) $\mathrm{m} / \mathrm{z}: 331(\mathrm{M}+, 100 \%)$.

\section{3-(2-oxo-2-phenylethylamino)-}

$\mathrm{N}$-phenylbenzamide (53)

Prepared by reacting $24(3 \mathrm{~g}, 14.1 \mathrm{mmol})$ and $\mathrm{H1}(2.81 \mathrm{~g}$, $14.1 \mathrm{mmol})$ in the presence of potassium carbonate $(1.41 \mathrm{~g}$, $14.1 \mathrm{mmol})$, to yield $2.7 \mathrm{~g}(58 \%)$ of 53 as yellow crystals. Melting point $204^{\circ} \mathrm{C}-205^{\circ} \mathrm{C}$; IR 3,381, 3,346, 3,062, 1,685, $1,654,1,363 \mathrm{~cm}^{-1} ;{ }^{1} \mathrm{H}$ NMR (400 mHz, DMSO-d6): $\delta 4.77$ $\left(\mathrm{d}, 2 \mathrm{H}, \mathrm{CH}_{2}, \mathrm{~J}=5.3 \mathrm{~Hz}\right), 6.14$ (t, 1H, NH-Ar, J=5.3 Hz), 6.91 (d, 1H, Ar-H, J=7.7 Hz), 7.09 (t, 1H, Ar-H, J=7.6 Hz), $7.16(\mathrm{~d}, 1 \mathrm{H}, \mathrm{Ar}-\mathrm{H}, \mathrm{J}=7.4 \mathrm{~Hz}), 7.24$ (m, 2H, Ar-H), 7.34 (t, 2H, Ar-H, J=7.8 Hz), 7.57 (t, 2H, Ar-H, J=7.5 Hz), 7.68 (t, 1H, Ar-H, J=7.3 Hz), 7.77 (d, 2H, Ar-H, J=8 Hz), 8.09 (d, 2H, Ar-H, J=7.6 Hz), 10.10 (s, 1H, CO-NH); ${ }^{13} \mathrm{C}-\mathrm{NMR}$ (DMSO-d6): $\delta 52.44,114.02,117.97,118.26,123.00,126.18$, 130.53, 131.21, 131.48, 136.27, 137.71, 138.53, 141.89, 150.87, 168.95, 199.19; MS (EI) m/z: 331 (M+, 100\%).

\section{4-(2-oxo-2-phenylethylamino)-}

\section{$\mathrm{N}$-p-tolylbenzamide (54)}

Prepared by reacting 25 (3.41 g, $15.1 \mathrm{mmol})$ and $\mathrm{H} 1$ (3 g, $15.1 \mathrm{mmol})$ in the presence of potassium carbonate $(1.51 \mathrm{~g}$, $15.1 \mathrm{mmol})$, to yield $1.35 \mathrm{~g} \mathrm{(26 \% )}$ of 54 as light yellow crystals. Melting point $198^{\circ} \mathrm{C}-199^{\circ} \mathrm{C}$; IR $3,400,3,057,1,678$, 1,658, $1,315 \mathrm{~cm}^{-1} ;{ }^{1} \mathrm{H}$ NMR (400 mHz, DMSO-d6): $\delta 2.25$ (s, 3H, $\left.\mathrm{CH}_{3}\right), 4.78$ (s, 2H, $\left.\mathrm{CH}_{2}\right), 6.6$ (s, 1H, NH-Ar), 6.74 (d, 2H, Ar-H, J=8.2 Hz), 7.1 (d, 2H, Ar-H, J=7.4 Hz), 7.59 (m, 4H, Ar-H), 7.65 (m, 1H, Ar-H), 7.75 (d, 2H, Ar-H, J=8 Hz), 8.08 (d, 2H, Ar-H, J=7.4 Hz), 9.72 (s, 1H, CO-NH); ${ }^{13} \mathrm{C}-\mathrm{NMR}$ (DMSO-d6): $\delta 20.95,49.97,111.94,120.75,122.29,128.41$, 129.34, 129.37, 129.58, 132.40, 134.17, 135.49, 137.58, 151.54, 165.64, 196.75; MS (EI) m/z: 345 (M+, 100\%).

\section{3-(2-oxo-2-phenylethylamino)-} $\mathrm{N}$-p-tolylbenzamide (55)

Prepared by reacting 26 (4.6 g, $20.3 \mathrm{mmol})$ and H1 (4.05 g, $20.3 \mathrm{mmol})$ in the presence of potassium carbonate $(2.81 \mathrm{~g}$,
$20.3 \mathrm{mmol})$, to yield $4.8 \mathrm{~g}(69 \%)$ of 55 as off-white crystals. Melting point $210^{\circ} \mathrm{C}-211^{\circ} \mathrm{C}$; IR 3,381, 3,062, 1,681, 1,651, 1,330 cm ${ }^{-1} ;{ }^{1} \mathrm{H}$ NMR (400 mHz, DMSO-d6): $\delta 2.27$ (s, $3 \mathrm{H}$, CH3), 4.79 (d, 2H, CH2, J = 3.2 Hz), 6.16 (s, 1H, NH-Ar), 6.91 (d, 1H, Ar-H, J = 7.8 Hz), 7.16 (d, 2H, Ar-H, J = 7.2 Hz), 7.22 (m, 3H, Ar-H), 7.59 (t, 2H, Ar-H, J =7.1 Hz), 7.66 (d, 2H, Ar-H, $\mathrm{J}=9.6 \mathrm{~Hz}), 7.69(\mathrm{t}, 1 \mathrm{H}, \mathrm{Ar}-\mathrm{H}, \mathrm{J}=7.8 \mathrm{~Hz}), 8.11(\mathrm{~d}, 2 \mathrm{H}, \mathrm{Ar}-\mathrm{H}$, $\mathrm{J}=7.3 \mathrm{~Hz}$ ), 10.02 (s,1H, CO-NH).; ${ }^{13} \mathrm{C}-\mathrm{NMR}$ (DMSO-d6): $\delta 19.65,48.98,110.53,114.42,114.66,119.49,127.06,127.90$, 127.98, 128.10, 131.54, 132.76, 134.25, 135.15, 135.94, 147.38, 165.21, 195.67; MS (EI) m/z: 345 (M+, 100\%).

\section{4-(2-oxo-2-phenylethylamino)- $\mathrm{N}$-m-tolylbenzamide (56)}

Prepared by reacting $27(3.41 \mathrm{~g}, 15.1 \mathrm{mmol})$ and $\mathrm{H1}(3 \mathrm{~g}$, $15.1 \mathrm{mmol})$ in the presence of potassium carbonate $(1.51 \mathrm{~g}$, $15.1 \mathrm{mmol})$, to yield $1.1 \mathrm{~g}(21 \%)$ of 56 as white crystals. Melting point $161^{\circ} \mathrm{C}-162^{\circ} \mathrm{C}$; IR 3,381, 3,292, 1,681, 1,658, 1,303 $\mathrm{cm}^{-1}$; ${ }^{1} \mathrm{H}$ NMR (400 mHz, DMSO-d6): $\delta 2.29$ (s, 3H, CH3), 4.79 (d, 2H, CH2, J = 5.1 Hz), 6.53 (t, 1H, NH-Ar, J =5 Hz), 6.76 (d, 2H, Ar-H, J = 8.3 Hz), 6.86 (d, 1H, Ar-H, J = 7.2 Hz), 7.18 (t, 1H, Ar-H, J=7.7 Hz), 7.56 (m, 2H, Ar-H), 7.69 (t, 1H, Ar-H, $\mathrm{J}=7.2 \mathrm{~Hz}), 7.79$ (d, 2H, Ar-H, J = 8.3 Hz), 8.09 (d, 2H, Ar-H, $\mathrm{J}=7.8 \mathrm{~Hz}$ ), 9.75 (s, 1H, CO-NH).; ${ }^{13} \mathrm{C}-\mathrm{NMR}$ (DMSO-d6): $\delta 21.87,50.17,112.17,118.12,121.54,122.54,124.42,128.57$, $128.98,129.50,129.83,134.31,135.70,138.27,140.23$, 151.78, 165.98, 196.90; MS (EI) m/z: 345 (M+, 100\%).

\section{3-(2-oxo-2-phenylethylamino)- $\mathrm{N}$-m-tolylbenzamide (57)}

Prepared by reacting $28(226.27 \mathrm{~g}, 8.4 \mathrm{mmol})$ and $\mathrm{H} 1(1.67 \mathrm{~g}$, $8.4 \mathrm{mmol})$ in the presence of potassium carbonate $(0.84 \mathrm{~g}$, $8.4 \mathrm{mmol})$, to yield $1.4 \mathrm{~g}(46 \%)$ of 57 as light yellow crystals. Melting point $177^{\circ} \mathrm{C}-178^{\circ} \mathrm{C}$; IR 3,365, 3,282, 3,059, 1,691, 1,643, 1,332 $\mathrm{cm}^{-1} ;{ }^{1} \mathrm{H}$ NMR (400 mHz, DMSO-d6): $\delta 2.46$ (s, $\left.3 \mathrm{H}, \mathrm{CH}_{3}\right), 4.95$ (s, $2 \mathrm{H}, \mathrm{CH}_{2}$ ), 3.57 (d, 1H, NH-Ar, J=4.6 Hz), 7.07 (s, 2H, Ar-H), 7.34 (d, 1H, Ar-H, J=3.9 Hz), 7.39 (m, 3H, Ar-H), 7.74 (m, 3H, Ar-H), 7.79 (s, 1H, Ar-H), 7.84 (t, 1H, Ar-H, J=5.8 Hz), 8.26 (d, 2H, Ar-H, J=4.4 Hz), 10.19 (s, 1H, CO-NH); ${ }^{13} \mathrm{C}-\mathrm{NMR}$ (DMSO-d6): $\delta$ 21.22, 49.85, 111.41 , $115.39,115.63,117.61,121.04,124.26,127.90,128.41$, $128.46,128.85,133.62,135.13,135.96,137.73,139.19$, 148.24, 166.24, 196.57; MS (EI) m/z: 345 (M+, 100\%).

\section{$\mathrm{N}$-(4-chlorophenyl)-4-(2-oxo-2-} phenylethylamino)benzamide (58)

Prepared by reacting 29 (4 g, $16.1 \mathrm{mmol})$ and $\mathrm{H} 1$ (3.2 g, $16.1 \mathrm{mmol})$ in the presence of potassium carbonate 
(1.6 $\mathrm{g}, 16.1 \mathrm{mmol})$, to yield $3 \mathrm{~g}(55 \%)$ of 58 as light yellow crystals. Melting point $220^{\circ} \mathrm{C}-222^{\circ} \mathrm{C}$; IR 3,392, 3,360, 3,041, 1,672, 1,651, 1,338, 1,089 $\mathrm{cm}^{-1} ;{ }^{1} \mathrm{H}$ NMR (400 mHz, DMSO-d6): $\delta 4.79$ (d, 2H, CH2), 6.56 (s, 1H, NH-Ar), 6.76 (s, 2H, Ar-H), 7.34 (s, 2H, Ar-H), 7.55 (s, 2H, Ar-H), 7.67 (s, 1H, Ar-H), 7.78 (s, 4H, Ar-H), 8.07 (s, 2H, Ar-H), 9.95 (s,1H, CO-NH).; ${ }^{13} \mathrm{C}-\mathrm{NMR}$ (DMSO-d6): $\delta$ 52.10, 114.13, 124.01, 124.28, 129.18, 130.55, 130.99, 131.46, 131.89, $136.29,137.62,141.32,153.92,168.04,198.79$; MS (EI) $\mathrm{m} / \mathrm{z}: 365(\mathrm{M}+, 100 \%)$.

\section{N-(4-chlorophenyl)-3-(2-oxo-2-}

phenylethylamino)benzamide (59)

Prepared by reacting 30 (2 g, $8 \mathrm{mmol})$ and $\mathrm{H} 1$ (1.6 g, $8 \mathrm{mmol})$ in the presence of potassium carbonate $(0.8 \mathrm{~g}, 8 \mathrm{mmol})$, to yield $2.12 \mathrm{~g}(89 \%)$ of 59 as yellow crystals. Melting point $230^{\circ} \mathrm{C}-233^{\circ} \mathrm{C}$; IR 3,398, 3,344, 3,061, 1,678, 1,647, 1,330, $1,093 \mathrm{~cm}^{-1}$; ${ }^{1} \mathrm{H}$ NMR (400 mHz, DMSO-d6): $\delta 4.79$ (d, 2H, $\mathrm{CH} 2), 6.56$ (s, 1H, NH-Ar), 6.76 (s, 2H, Ar-H), 7.34 (s, 2H, Ar-H), 7.55 (s, 2H, Ar-H), 7.67 (s, 1H, Ar-H), 7.78 (s, 4H, Ar-H), 8.07 (s, 2H, Ar-H), 9.95 (s,1H, CO-NH).; ${ }^{13} \mathrm{C}-\mathrm{NMR}$ (DMSO-d6): $\delta 49.77,111.39,115.25,115.69,121.75,127.03$, $127.88,128.45,128.81,133.59,135.07,135.62,138.29$, 148.25, 166.38, 196.49; MS (EI) m/z: 365 (M+, 100\%).

\section{N-(3-chlorophenyl)-4-(2-oxo-2-}

\section{phenylethylamino)benzamide (60)}

Prepared by reacting $31(3.4 \mathrm{~g}, 13.8 \mathrm{mmol})$ and $\mathrm{H} 1$ (2.74 g, $13.8 \mathrm{mmol})$ in the presence of potassium carbonate $(1.37 \mathrm{~g}$, $13.8 \mathrm{mmol})$, to yield $1.2 \mathrm{~g}(24 \%)$ of 60 as white crystals. Melting point $183^{\circ} \mathrm{C}-185^{\circ} \mathrm{C}$; IR 3,381, 3,302, 3,047, 1,681, 1,658, 1,313, 1,097 $\mathrm{cm}^{-1}$; ${ }^{1} \mathrm{H}$ NMR (400 mHz, DMSO-d6): $\delta 4.77$ (s, 2H, CH2), 6.15 (s, 1H, NH-Ar), 6.91 (s, 1H, Ar-H), 7.2 (m, 3H, Ar-H), 7.39 (d, 2H, Ar-H, J =6.7 Hz), 7.57 (s, 2H, Ar-H), 7.69 (s, 1H, Ar-H), 7.8 (d, 2H, Ar-H, J = 6.6 Hz), 8.09 (d, 2H, Ar-H, J = 4.6 Hz), 10.21 (s, 1H, CO-NH).; ${ }^{13} \mathrm{C}-\mathrm{NMR}$ (DMSO-d6): $\delta 88.21,111.78,117.53,118.59,121.73,127.61$, $128.22,128.36,128.47,128.61,129.30,132.1,132.47,132.8$, 140.09, 164.87, 194.89; MS (EI) m/z: 365 (M+, 100\%).

\section{N-(3-chlorophenyl)-3-(2-oxo-2-}

phenylethylamino)benzamide (6I)

Prepared by reacting $32(3.7 \mathrm{~g}, 15.1 \mathrm{mmol})$ and $\mathrm{H} 1$ ( $3 \mathrm{~g}, 15.1 \mathrm{mmol})$ in the presence of potassium carbonate ( $1.5 \mathrm{~g}, 15.1 \mathrm{mmol})$, to yield $2 \mathrm{~g}(36 \%)$ of 61 as bright yellow crystals. Melting point $192^{\circ} \mathrm{C}-193^{\circ} \mathrm{C}$; IR 3,363, 3,277, 3,066, 1,691, 1,643, 1,311 $\mathrm{cm}^{-1}$; ${ }^{1} \mathrm{H}$ NMR (400 mHz, DMSO-d6): $\delta 4.76\left(\mathrm{~d}, 2 \mathrm{H}, \mathrm{CH}_{2}, \mathrm{~J}=5.4 \mathrm{~Hz}\right), 6.16(\mathrm{t}, 1 \mathrm{H}, \mathrm{NH}-\mathrm{Ar}, \mathrm{J}=5.3 \mathrm{~Hz})$,
6.93 (d, 1H, Ar-H, J=7.9 Hz), 7.14 (d, 2H, Ar-H, J=7.7 Hz), 7.18 (s, 1H, Ar-H), 7.23 (t, 2H, Ar-H, J=7.8 Hz), 7.36 (t, 1H, Ar-H, J=8.1 Hz), 7.57 (t, 2H, Ar-H, J=7.5 Hz), $7.68(\mathrm{t}$, 2H, Ar-H, J=7.2 Hz), 7.95 (s, 1H, Ar-H), 8.08 (d, 2H, Ar-H, $\mathrm{J}=7.2 \mathrm{~Hz}$ ), 10.25 (s, 1H, CO-NH); ${ }^{13} \mathrm{C}-\mathrm{NMR}$ (DMSO-d6): $\delta 48.95,110.58,114.43,114.97,117.69,118.75,122.29$, $127.05,127.98,129.42,132.06,132.75,134.67,139.97$, 147.45, 165.69, 195.64; MS (EI) m/z: 365 (M+, 100\%).

N-(4-methoxyphenyl)-4-(2-oxo-2-

phenylethylamino)benzamide (62)

Prepared by reacting $33(3.6 \mathrm{~g}, 15.1 \mathrm{mmol})$ and $\mathrm{H} 1 \mathrm{(3 \textrm {g } \text { , }}$ $15.1 \mathrm{mmol})$ in the presence of potassium carbonate $(1.5 \mathrm{~g}$, $15.1 \mathrm{mmol})$, to yield $2.1 \mathrm{~g}(39 \%)$ of 62 as off-white crystals. Melting point $203^{\circ} \mathrm{C}-205^{\circ} \mathrm{C}$; IR $3,367,3,315$, $3,045,1,685,1,639,1,313,1,253,1,033 \mathrm{~cm}^{-1} ;{ }^{1} \mathrm{H}$ NMR (400 mHz, DMSO-d6): $\delta 3.14\left(\mathrm{~s}, 3 \mathrm{H}, \mathrm{CH}_{3}\right), 4.78(\mathrm{~s}, 2 \mathrm{H}$, $\left.\mathrm{CH}_{2}\right), 6.50$ (s, 1H, NH-Ar), 6.75 (d, 2H, Ar-H, J=6.8 Hz), $6.88(\mathrm{~d}, 2 \mathrm{H}, \mathrm{Ar}-\mathrm{H}, \mathrm{J}=7.3 \mathrm{~Hz}), 7.56(\mathrm{~m}, 2 \mathrm{H}, \mathrm{Ar}-\mathrm{H}), 7.63$ (d, 2H, Ar-H, J=7.6 Hz), 7.68 (s, 1H, Ar-H), 7.77 (d, 2H, Ar-H, J=6.8 Hz), 8.08 (d, 2H, Ar-H, J=5.9 Hz), 9.72 (s, 1h, CO-NH); ${ }^{13} \mathrm{C}-\mathrm{NMR}$ (DMSO-d6): $\delta 50.17,55.82,112.16$, 114.32, 122.62, 128.57, 129.51, 129.71, 133.35, 134.33, 135.68, 151.66, 155.87, 165.73, 196.94; MS (EI) m/z: 361 $(\mathrm{M}+, 100 \%)$.

$\mathrm{N}$-(4-methoxyphenyl)-3-(2-oxo-2phenylethylamino)benzamide (63)

Prepared by reacting $34(1 \mathrm{~g}, 4.1 \mathrm{mmol})$ and $\mathrm{H} 1(0.82 \mathrm{~g}$, $4.1 \mathrm{mmol})$ in the presence of potassium carbonate $(0.5 \mathrm{~g}$, $4.1 \mathrm{mmol})$, to yield $0.9 \mathrm{~g}(61 \%)$ of 63 as bright yellow crystals. Melting point $191^{\circ} \mathrm{C}-193^{\circ} \mathrm{C}$; IR 3,365, 3,269, 3,039, 1,708, 1,647, 1,359, 1,082 cm ${ }^{-1}$; ${ }^{1} \mathrm{H}$ NMR $(400 \mathrm{mHz}$, DMSOd6): $\delta 3.74$ (s, 3H, Ar-H), 4.77 (d, 2H, $\mathrm{CH}_{2}$, J=4.9 Hz), 6.12 (t, 1H, NH-Ar, J=4.6 Hz), 6.9 (t, 3H, Ar-H, J=8.7 Hz), 7.13 (d, 1H, Ar-H, J=7.3 Hz), 7.18 (S, 1H, Ar-H), 7.22 $(\mathrm{d}, 1 \mathrm{H}, \mathrm{Ar}-\mathrm{H}, \mathrm{J}=7.5 \mathrm{~Hz}), 7.58(\mathrm{t}, 2 \mathrm{H}, \mathrm{Ar}-\mathrm{H}, \mathrm{J}=7.3 \mathrm{~Hz})$, 7.65 (d, 2H, Ar-H, J=8.8 Hz), 7.7 (d, 2H, Ar-H, J=7.4 Hz), 8.09 (d, 2H, Ar-H, J=7.6 Hz), 9.95 (s, 1H, CO-NH); ${ }^{13} \mathrm{C}-\mathrm{NMR}$ (DMSO-d6): $\delta 50.32,55.68,111.82,114.19,115.74,115.92$, $122.47,128.39,129.25,129.33,133.22,134.10,135.59$, 136.47, 148.81, 156.11, 166.38, 197.06; MS (EI) m/z: 361 $(\mathrm{M}+, 100 \%)$.

\section{$\mathrm{N}$-(3-methoxyphenyl)-4-(2-oxo-2-}

\section{phenylethylamino)benzamide (64)}

Prepared by reacting 35 (3.65 g, $15.1 \mathrm{mmol})$ and $\mathrm{H} 1$ $(3 \mathrm{~g}, 15.1 \mathrm{mmol})$ in the presence of potassium carbonate 
(1.51 g, $15.1 \mathrm{mmol})$, to yield $1.9 \mathrm{~g}(35 \%)$ of 64 as light yellow crystals. Melting point $173^{\circ} \mathrm{C}-175^{\circ} \mathrm{C}$; IR 3,377, 3,294, 3,059, 1,687, 1,643, 1,309, 1,280, 1,037 $\mathrm{cm}^{-1} ;{ }^{1} \mathrm{H}$ NMR (400 mHz, DMSO-d6): $\delta 3.74\left(\mathrm{~s}, 3 \mathrm{H}, \mathrm{CH}_{3}\right), 4.8$ (d, $\left.2 \mathrm{H}, \mathrm{CH}_{2}, \mathrm{~J}=5.3 \mathrm{~Hz}\right), 6.56(\mathrm{t}, 2 \mathrm{H}, \mathrm{NH}-\mathrm{Ar}, \mathrm{J}=5.2 \mathrm{~Hz})$, 6.63 (d, 1H, Ar-H, J=7.9 Hz), 6.77 (d, 2H, Ar-H, J=8.4 Hz), 7.21 (t, 1H, Ar-H, J=8.1 Hz), 7.35 (d, 1H, Ar-H, J=8.1 Hz), 7.47 (s, 1H, Ar-H), 7.58 (t, 2H, Ar-H, J=7.5 Hz), 7.7 (t, 1H, Ar-H, J=7.3 Hz), 7.79 (d, 2H, Ar-H, J=8.4 Hz), 8.09 (d, 2H, Ar-H, J=7.6 Hz), 9.81 (s, 1H, CO-NH); ${ }^{13} \mathrm{C}-$ NMR (DMSO-d6): $\delta 52.1,57.57,108.48,111.16,114.12$, $115.05,124.34,130.55,131.47,131.84,136.31,137.62$, $143.53,153.82,162.03,168.03,198.84 ; \mathrm{MS}(\mathrm{EI}) \mathrm{m} / \mathrm{z}$ : $361(\mathrm{M}+, 100 \%)$.

\section{$\mathrm{N}$-(3-methoxyphenyl)-3-(2-oxo-2-}

\section{phenylethylamino)benzamide (65)}

Prepared by reacting 36 (3.65 g, $15.1 \mathrm{mmol})$ and $\mathrm{H} 1$ (3 g, $15.1 \mathrm{mmol})$ in the presence of potassium carbonate $(1.51 \mathrm{~g}$, $15.1 \mathrm{mmol})$, to yield $1.62 \mathrm{~g}(30 \%)$ of 65 as beige crystals. Melting point $174^{\circ} \mathrm{C}-176^{\circ} \mathrm{C}$; IR 3,365, 3,305, 3,057, 1,693, 1,647, 1,319, 1,265, 1,035 cm ${ }^{-1}$; ${ }^{1} \mathrm{H}-\mathrm{NMR}$ (400 mHz, DMSOd6): $\delta 3.74\left(\mathrm{~s}, 3 \mathrm{H}, \mathrm{CH}_{3}\right), 4.76\left(\mathrm{~d}, 2 \mathrm{H}, \mathrm{CH}_{2}, \mathrm{~J}=2.8 \mathrm{~Hz}\right), 6.13(\mathrm{~s}$, 1H, NH-Ar), 6.66 (d, 1H, Ar-H, J=7.5 Hz), 6.9 (d, 1H, Ar-H, $\mathrm{J}=7.2 \mathrm{~Hz}$ ), 7.9 (d, 1H, Ar-H, J=7.2 Hz), 7.18 (s, 1H, Ar-H), 7.22 (t, 2H, Ar-H, J=7.5 Hz), 7.34 (d, 1H, Ar-H, J=7.3 Hz), 7.47 (s, 1H, Ar-H), 7.57 (t, 2H, Ar-H, J=6.3 Hz), 7.66 (t, 1H, Ar-H, J=6.3 Hz), 8.09 (d, 2H, Ar-H, J=7.1 Hz), 10.03 (s, 1H, CO-NH); ${ }^{13} \mathrm{C}-\mathrm{NMR}$ (DMSO-d6): $\delta 45.03,50.20,101.21$, $104.17,106.62,107.73,110.47,110.81,123.10,123.96$, $124.02,124.50,128.79,130.33,131.12,135.71,143.44$, 154.62, 161.49, 191.72; MS (EI) m/z: $361(\mathrm{M}+, 100 \%)$.

\section{$\mathrm{N}$-(4-acetylphenyl)-4-(2-oxo-2-}

\section{phenylethylamino)benzamide (66)}

Prepared by reacting $37(3 \mathrm{~g}, 11.8 \mathrm{mmol})$ and $\mathrm{H} 1$ (2.35 g, $11.8 \mathrm{mmol})$ in the presence of potassium carbonate $(1.18 \mathrm{~g}$, $11.8 \mathrm{mmol})$, to yield $3.3 \mathrm{~g}(75 \%)$ of 66 as white crystals. Melting point $243^{\circ} \mathrm{C}-245^{\circ} \mathrm{C}$; IR $3,390,3,369,3,001$, 1,666, 1,651, 1,323 cm ${ }^{-1} ;{ }^{1} \mathrm{H}-\mathrm{NMR}$ (400 mHz, DMSO-d6): $\delta 2.52\left(\mathrm{~s}, 3 \mathrm{H}, \mathrm{CH}_{3}\right), 4.80\left(\mathrm{~s}, 2 \mathrm{H}, \mathrm{CH}_{2}\right), 6.76(\mathrm{~d}, 2 \mathrm{H}, \mathrm{Ar}-\mathrm{H}$, $\mathrm{J}=8.6 \mathrm{~Hz}), 7.57$ (t, 2H, Ar-H, J=7.6 Hz), 7.68 (t, 1H, Ar-H, $\mathrm{J}=7.4 \mathrm{~Hz}), 7.8$ (d, 2H, Ar-H, J=8.6 Hz), 7.91 (m, 4H, Ar-H), 8.08 (d, 2H, Ar-H, J=7.4 Hz), 10.13 (s, 1H, NH-CO); ${ }^{13} \mathrm{C}-\mathrm{NMR}$ (DMSO-d6): $\delta$ 29.04, 52.09, 114.13, 121.73, 123.80, 130.55, 131.44, 131.68, 132.07, 133.99, 136.27, 137.64, 146.95, 154.12, 168.25, 198.71, 199.14; MS (EI) m/z: $737(\mathrm{M}+, 100 \%)$.
$\mathrm{N}$-(4-acetylphenyl)-3-(2-oxo-2-

phenylethylamino)benzamide (67)

Prepared by reacting 38 ( $3 \mathrm{~g}, 11.8 \mathrm{mmol})$ and $\mathrm{H} 1$ (2.35 g, $11.8 \mathrm{mmol})$ in the presence of potassium carbonate $(1.18 \mathrm{~g}$, $11.8 \mathrm{mmol})$, to yield $3.1 \mathrm{~g}(70 \%)$ of 67 as light yellow crystals. Melting point $242^{\circ} \mathrm{C}-244^{\circ} \mathrm{C}$; IR 3,396, 3,338, 3,059, 1,666, 1,651,1,330 $\mathrm{cm}^{-1}$; ${ }^{1} \mathrm{H}-\mathrm{NMR}(400 \mathrm{mHz}$, DMSO-d6): $\delta 2.54\left(\mathrm{~s}, 3 \mathrm{H}, \mathrm{CH}_{3}\right), 4.77\left(\mathrm{~s}, 2 \mathrm{H}, \mathrm{CH}_{2}\right)$, 6.16 (s, 1H, NH-Ar), 6.91 (s, 1H, Ar-H), $7.16(\mathrm{~d}, 1 \mathrm{H}$, Ar-H, J=7.6 Hz), 7.24 (t, 1H, Ar-H, J=7.6 Hz), 7.58 (t, 2H, Ar-H, J=7.3 Hz), 7.67 (t, 1H, Ar-H, J=7.3 Hz), 7.93 (m, 4H, Ar-H), 8.08 (d, 2H, Ar-H, J=7.5 Hz), 10.40 (s, 1H, CO-NH); ${ }^{13} \mathrm{C}-\mathrm{NMR}$ (DMSO-d6): $\delta 25.60,48.93,110.63$, 114.54, 115.04, 118.52, 127.03, 127.98, 128.40, 131.2, $132.75,134.23,134.8,147.44,165.86,195.78$; MS (EI) $\mathrm{m} / \mathrm{z}: 373(\mathrm{M}+, 100 \%)$.

\section{$\mathrm{N}$-(3-acetylphenyl)-4-(2-oxo-2-}

phenylethylamino)benzamide (68)

Prepared by reacting 39 ( $3 \mathrm{~g}, 11.8 \mathrm{mmol})$ and $\mathrm{H} 1$ (2.35 g, $11.8 \mathrm{mmol})$ in the presence of potassium carbonate $(1.18 \mathrm{~g}$, $11.8 \mathrm{mmol})$, to yield $2.5 \mathrm{~g}(68 \%)$ of 68 as off-white crystals. Melting point $201{ }^{\circ} \mathrm{C}-203^{\circ} \mathrm{C}$; IR 3,369, 3,294, 3,072, 1,687, 1,643, 1,309 $\mathrm{cm}^{-1}$; ${ }^{1} \mathrm{H}$ NMR (400 mHz, DMSO-d6): $\delta 2.57\left(\mathrm{~s}, 3 \mathrm{H}, \mathrm{CH}_{3}\right), 4.81\left(\mathrm{~d}, 2 \mathrm{H}, \mathrm{CH}_{2}, \mathrm{~J}=5.4 \mathrm{~Hz}\right), 6.59$ (t, 1H, NH-Ar, J=5.3 Hz), 6.79 (d, 2H, Ar-H, J=8.7 Hz), 7.47 (t, 1H, Ar-H, J=7.9 Hz), 7.57 (t, 2H, Ar-H, J=7.6 Hz), 7.67 (m, 2H, Ar-H), 7.83 (d, 2H, Ar-H, J=8.7 Hz), 8.08 (m, 3H, Ar-H), 8.36 (s, 1H, Ar-H), 10.04 (s, 1H, CO-NH); ${ }^{13} \mathrm{C}-\mathrm{NMR}$ (DMSO-d6): $\delta 29.38,52.10,114.15,122.16,123.94,125.61$, 127.27, 130.57, 131.54, 131.92, 136.31, 137.31, 139.80, 142.75, 153.97, 168.14, 198.81, 200.50; MS (EI) m/z: 373 $(\mathrm{M}+, 100 \%)$.

\section{$\mathrm{N}$-(4-acetylphenyl)-3-(2-oxo-2-}

phenylethylamino)benzamide (69)

Prepared by reacting $40(1.5 \mathrm{~g}, 5.9 \mathrm{mmol})$ and $\mathrm{H} 1(1.18 \mathrm{~g}$, $5.9 \mathrm{mmol})$ in the presence of potassium carbonate $(0.55 \mathrm{~g}$, $5.9 \mathrm{mmol})$, to yield $1.1 \mathrm{~g}(55 \%)$ of 69 as white crystals. Melting point $182^{\circ} \mathrm{C}-184^{\circ} \mathrm{C}$; IR 3,387, 3,361, 3,064, 1,681, 1,651, 1,334 $\mathrm{cm}^{-1} ;{ }^{1} \mathrm{H}$ NMR (400 mHz, DMSO-d6): $\delta 2.58$ $\left(\mathrm{s}, 3 \mathrm{H}, \mathrm{CH}_{3}\right), 4.78\left(\mathrm{~s}, 2 \mathrm{H}, \mathrm{CH}_{2}\right), 6.16(\mathrm{~s}, 1 \mathrm{H}, \mathrm{Ar}-\mathrm{H}), 6.92$ (d, 1H, Ar-H, J=7.5 Hz), 7.18 (d, 1H, Ar-H, J=7 Hz), 7.22 (m, 2H, Ar-H), 7.49 (t, 1H, Ar-H, J=7.6 Hz), 7.57 (t, 2H, Ar-H, $\mathrm{J}=7 \mathrm{~Hz}), 7.68$ (t, 2H, Ar-H, J=7.2 Hz), 8.06 (d, 1H, Ar-H, $\mathrm{J}=7.2 \mathrm{~Hz}$ ), 8.09 (d, 2H, Ar-H, J=7.4 Hz), 8.37 (s, 1H, Ar-H), 10.28 (s, 1H, CO-NH); ${ }^{13} \mathrm{C}-\mathrm{NMR}$ (DMSO-d6): $\delta 27.25$, 50.30, 111.92, 115.81, 116.31, 120.14, 123.96, 125.25, 
$128.40,129.33,129.49,134.11,135.60,136.03,137.73$, 140.19, 148.79, 166.94, 197.01, 198.21; MS (EI) m/z: 373 $(\mathrm{M}+, 100 \%)$.

\section{Ethyl 4-[4-(2-oxo-2-phenylethylamino) benzoylamino]benzoate (70)}

Prepared by reacting 41 ( $3 \mathrm{~g}, 10.6 \mathrm{mmol})$ and $\mathrm{H} 1(2.1 \mathrm{~g}$, $10.6 \mathrm{mmol})$ in the presence of potassium carbonate $(1.5 \mathrm{~g}$, $10.6 \mathrm{mmol})$, to yield $1.7 \mathrm{~g}(43 \%)$ of 70 as light yellow crystals. Melting point $212^{\circ} \mathrm{C}-213^{\circ} \mathrm{C}$; IR 3,383, 3,061, 1,697, 1,681, 1,336 cm ${ }^{-1} ;{ }^{1} \mathrm{H}$ NMR (400 mHz, DMSO-d6): $\delta 1.31$ (t, $3 \mathrm{H}, \mathrm{CH}_{3}, \mathrm{~J}=8 \mathrm{~Hz}$ ), 4.27 (q, 2H, COO-CH $2, \mathrm{~J}=8 \mathrm{~Hz}$ ), 4.81 (d, 2H, $\left.\mathrm{CH}_{2}-\mathrm{N}-\mathrm{Ar}, \mathrm{J}=4 \mathrm{~Hz}\right), 6.62$ (t, 1H, NH-Ar, J=4 Hz), 6.79 (d, 2H, Ar-H, J=8 Hz), 7.54 (t, 2H, Ar-H, J=8 Hz), 7.67 (t, 2H, Ar-H, J=8 Hz), 7.82 (d, 2H, Ar-H, J=8 Hz), 7.92 (s, 4H, Ar-H), 8.09 (d, 2H, Ar-H, J=8 Hz), 10.14 (s, 1H, CO-NH); ${ }^{13} \mathrm{C}-\mathrm{NMR}$ (DMSO-d6): $\delta$ 16.57, 52.11, 63.00, 114.15, 121.88, 123.83, $126.46,130.58,131.45,132.09,132.63,136.28,137.65$, 146.96, 154.12, 168.10, 168.28, 198.72; MS (EI) m/z: 403 $(\mathrm{M}+, 100 \%)$.

\section{Ethyl 4-[3-(2-ixo-2-phenylethylamino)} benzoylamino]benzoate (7I)

Prepared by reacting $42(1.5 \mathrm{~g}, 5.3 \mathrm{mmol})$ and $\mathrm{H} 1$ (1.05 g, $5.3 \mathrm{mmol})$ in the presence of potassium carbonate $(0.75 \mathrm{~g}$, $5.3 \mathrm{mmol}$ ), to yield $0.9 \mathrm{~g}$ (43\%) of 71 as light yellow crystals. Melting point $180^{\circ} \mathrm{C}-182^{\circ} \mathrm{C}$; IR 3,408, 3,360, 2,981, 1,703, 1,654, 1,336 cm ${ }^{-1} ;{ }^{1} \mathrm{H}$ NMR (400 mHz, DMSO-d6): $\delta 3.93$ (s, 3H, $\left.\mathrm{CH}_{3}\right), 4.94$ (d, 2H, CH, J=5 Hz), 6.25 (t, 1H, NH-Ar, $\mathrm{J}=5 \mathrm{~Hz}), 6.92$ (m, 3H, Ar-H), 7.16 (d, 1H, Ar-H, J=7.2 Hz), 7.25 (d, 1H Ar-H, J=7.2 Hz), 7.57 (t, 2H, Ar-H, J=7 Hz), 7.7.67 (d, 2H, Ar-H, J=7.1 Hz), 8.18 (d, 2H, Ar-H, J=7 Hz), 10.23 (s, $1 \mathrm{H}, \mathrm{CO}-\mathrm{NH}$ ); ${ }^{13} \mathrm{C}-\mathrm{NMR}$ (DMSO-d6): $\delta 21.22,52.35$, $58.71,114.16,124.49,124.91,125.61,129.57,131.82$, $133.19,133.66,138.83,139.69,142.37,153.72,167.95$, 197.95 ; MS (EI) m/z: 403 (M+, 100\%).

\section{Ethyl 3-[4-(2-oxo-2-phenylethylamino) benzoylamino]benzoate (72)}

Prepared by reacting 43 ( $2 \mathrm{~g}, 7 \mathrm{mmol})$ and $\mathrm{H} 1$ (1.4 g, $7 \mathrm{mmol})$ in the presence of potassium carbonate $(1 \mathrm{~g}$, $7 \mathrm{mmol})$, to yield $1.35 \mathrm{~g}(48 \%)$ of 72 as light orange crystals. Melting point $221^{\circ} \mathrm{C}-223^{\circ} \mathrm{C}$; IR 3,379, 3,275, $3,049,1,714,1,645,1,361 \mathrm{~cm}^{-1}$; ${ }^{1} \mathrm{H}$ NMR $(400 \mathrm{mHz}$, DMSO-d6): $\delta 1.27$ (t, 3H, $\left.\mathrm{CH}_{3}, \mathrm{~J}=8 \mathrm{~Hz}\right), 4.26$ (q, 2H, COO$\left.\mathrm{CH}_{2}\right), 4.74$ (s, 2H, $\left.\mathrm{CH}_{2}-\mathrm{N}-\mathrm{Ar}\right), 6.51$ (s, 1H, NH-Ar), 6.71 (s, 2H, Ar-H), 7.41 (s, 1H, Ar-H), 7.56 (m, 4H, Ar-H), 7.75 (s, 2H, Ar-H), 8.01 (s, 3H, Ar-H), 8.35 (s, 1H, Ar-H),
9.99 (s, 1H, CO-NH); ${ }^{13} \mathrm{C}-\mathrm{NMR}$ (DMSO-d6): $\delta 14.16$, $49.45,60.68,111.45,120.58,121.28,123.50,124.48$, $124.80,127.88,128.77,129.23,130.18,133.23,135.00$, 140.08, 151.28, 165.40, 165.71, 196.10; MS (EI) m/z: $403(\mathrm{M}+, 100 \%)$.

\section{Ethyl 3-[3-(2-oxo-2-phenylethylamino)}

\section{benzoylamino] benzoate (73)}

Prepared by reacting 44 (1.5 g, $5.3 \mathrm{mmol})$ and H1 (1.05 g, $5.3 \mathrm{mmol})$ in the presence of potassium carbonate $(0.75 \mathrm{~g}$, $5.3 \mathrm{mmol})$, to yield $1.2 \mathrm{~g}(57 \%)$ of 73 as light yellow crystals. Melting point $194^{\circ} \mathrm{C}-195^{\circ} \mathrm{C}$; IR 3,365, 3,269, 3,068, 1,710, 1,645, 1,359 $\mathrm{cm}^{-1}$; ${ }^{1} \mathrm{H}$ NMR (400 mHz, DMSO-d6): $\delta 1.34$ (t, 3H, $\mathrm{CH}_{3}, \mathrm{~J}=6.9 \mathrm{~Hz}$ ), 4.34 (q, 2H, COO-CH $2, \mathrm{~J}=7 \mathrm{~Hz}$ ), 4.79 (d, $2 \mathrm{H}, \mathrm{CH}_{2}-\mathrm{N}-\mathrm{Ar}, \mathrm{J}=4.9 \mathrm{~Hz}$ ), 6.16 (t, 1H, NH-Ar, J=5 Hz), 7.22 (m, 3H, Ar-H), 7.5 (t, 1H, Ar-H, J=7.8 Hz), 7.59 (t, 2H, Ar-H, J=7.3 Hz), 7.69 (d, 2H, Ar-H, J=7.3 Hz), 8.70 (d, 2H, Ar-H, J=7.8 Hz), 8.1 (d, 2H, Ar-H, J=7.4 Hz), 8.44 (s, 1H, Ar-H), 10.30 (s, 1H, CO-NH); ${ }^{13} \mathrm{C}-\mathrm{NMR}$ (DMSO-d6): $\delta$ 14.12, 49.75, 60.70, 111.38, 115.27, 115.75, 120.71, $123.99,124.62,127.81,128.74,128.90,130.22$, 133.51, $135.05,135.43,139.60,148.20,165.59,166.36,196.43 ; \mathrm{MS}$ (EI) $\mathrm{m} / \mathrm{z}: 403(\mathrm{M}+, 100 \%)$.

\section{4-[2-(4-nitrophenyl)-2-oxoethylamino]- $\mathrm{N}$-phenylbenzamide (74)}

Prepared by reacting $23(1.4 \mathrm{~g}, 6.6 \mathrm{mmol})$ and $45(1.6 \mathrm{~g}$, $6.6 \mathrm{mmol})$ in the presence of potassium carbonate $(0.66 \mathrm{~g}$, $6.6 \mathrm{mmol})$, to yield $1.4 \mathrm{~g}(58 \%)$ of 74 as bright yellow crystals. Melting point $210^{\circ} \mathrm{C}-212^{\circ} \mathrm{C}$; IR 3,421, 3,402, 3,346, $3,062,1,680,1,653,1,333,1,520 \mathrm{~cm}^{-1}$; ${ }^{1} \mathrm{H}$ NMR $(400 \mathrm{mHz}$, DMSO-d6): $\delta 4.87$ (d, 2H, $\mathrm{CH}_{2}, \mathrm{~J}=8 \mathrm{~Hz}$ ), 6.60 (s, 1H, NH-Ar), 6.78 (d, 2H, Ar-H, J=8 Hz), 7.03 (t, 1H, Ar-H, J=8 Hz), 7.31 (t, 2H, Ar-H, J=12 Hz), 7.75 (d, 2H, Ar-H, J=8 Hz), 7.81 (d, 2H, Ar-H, J=8 Hz), 8.29 (d, 2H, Ar-H, J=8 Hz), 8.38 (d, 2H, $\mathrm{Ar}-\mathrm{H}, \mathrm{J}=8 \mathrm{~Hz}$ ), 9.82 (s, 1H, CO-NH); ${ }^{13} \mathrm{C}-\mathrm{NMR}$ (DMSO-d6): $\delta 45.38,106.74,115.42,117.21,118.21,119.11,123.70$, $124.41,124.68,134.95,134.99,145.41,146.26,160.49$, 191.02; MS (EI) m/z: 376 (M+, 100\%).

\section{4-[2-(3-methoxyphenyl)-2-oxoethylamino]- N-phenylbenzamide (75)}

Prepared by reacting $23(1.11 \mathrm{~g}, 5.2 \mathrm{mmol})$ and $46(1.2 \mathrm{~g}$, $5.2 \mathrm{mmol})$ in the presence of potassium carbonate $(0.52 \mathrm{~g}$, $5.2 \mathrm{mmol})$, to yield $0.9 \mathrm{~g}(47 \%)$ of 75 as light yellow crystals. Melting point $200^{\circ} \mathrm{C}-202^{\circ} \mathrm{C}$; IR 3,379, 3,329, 3,051, 1,685, 1,647, 1,311, 1,267, 1,026 $\mathrm{cm}^{-1}$; ${ }^{1} \mathrm{H}$ NMR $(400 \mathrm{mHz}$, DMSO-d6): $\delta 3.83$ (s, 3H, $\mathrm{CH}_{3}$ ), 4.79 (d, 2H, $\mathrm{CH}_{2}, \mathrm{~J}=4 \mathrm{~Hz}$ ), 
$6.54(\mathrm{t}, 1 \mathrm{H}, \mathrm{NH}-\mathrm{Ar}, \mathrm{J}=4 \mathrm{~Hz}), 6.78$ (d, 2H, Ar-H, J=8 Hz), 7.03 (t, 1H, Ar-H, J=8 Hz), 7.25 (d, 1H, Ar-H, J=8 Hz), 7.30 (t, 2H, Ar-H, J=8 Hz), 7.48 (t, 1H, Ar-H, J=8 Hz), 7.57 (s, 1H, Ar-H), 7.69 (d, 1H, Ar-H, J=8 Hz), 7.77 (d, 2H, Ar-H, $\mathrm{J}=8 \mathrm{~Hz}$ ), 7.80 (d, 2H, Ar-H, J=8 Hz), 9.83 (s, 1H, CO-NH); ${ }^{13} \mathrm{C}-\mathrm{NMR}$ (DMSO-d6): $\delta$ 52.25, 58.05, 114.13, 115.28, $122.16,122.83,122.99,124.43,125.60,131.11,131.63$, $132.64,139.04,142.39,153.79,153.79,162.13,167.95$, 198.65; MS (EI) m/z: $361(\mathrm{M}+, 100 \%)$.

\section{4-[2-(4-methoxyphenyl)-2-oxoethylamino]-} N-phenylbenzamide (76)

Prepared by reacting $23(1 \mathrm{~g}, 4.7 \mathrm{mmol})$ and 47 (1.08 g, $4.7 \mathrm{mmol})$ in the presence of potassium carbonate $(0.47 \mathrm{~g}$, $4.7 \mathrm{mmol})$, to yield $0.9 \mathrm{~g}(53 \%)$ of 76 as off-white crystals. Melting point $206^{\circ} \mathrm{C}-208^{\circ} \mathrm{C}$; IR 3,383, 3,282, 3,049, 1670 , 1,641, 1,321, 1,029 $\mathrm{cm}^{-1} ;{ }^{1} \mathrm{H}$ NMR (400 mHz, DMSO-d6): $\delta 3.88\left(\mathrm{~s}, 3 \mathrm{H}, \mathrm{CH}_{3}\right), 4.73(\mathrm{~d}, 2 \mathrm{H}, \mathrm{Ar}-\mathrm{H}, \mathrm{J}=5 \mathrm{~Hz}), 6.4921$ (t, 1H, NH-Ar, J=5 Hz), 6.77 (d, 2H, Ar-H, J=8.3 Hz), 7.05 (t, 1H, Ar-H, J=7.3 Hz), 7.1 (d, 2H, Ar-H, J=8.4 Hz), 7.32 (t, 2H, Ar-H, J=7.5 Hz), 7.77 (d, 2H, Ar-H, J=8.4 Hz), 7.8 (d, 2H, Ar-H, J=8.3 Hz), 8.09 (d, 2H, Ar-H, J=8.4 Hz), 9.81 (s, $1 \mathrm{H}, \mathrm{CO}-\mathrm{NH}) ;{ }^{13} \mathrm{C}-\mathrm{NMR}$ (DMSO-d6): $\delta 49.07,55.54,111.39$, $113.96,120.11,121.66,122.87,127.88,128.38,129.09$, 130.20, 139.67, 151.67, 151.09, 163.42, 165.21, 194.33; MS (EI) $\mathrm{m} / \mathrm{z}: 361(\mathrm{M}+, 100 \%)$.

\section{4-[2-(4-cyanophenyl)-2-oxoethylamino]-}

N-phenylbenzamide (77)

Prepared by reacting 23 (1.89 g, $8.9 \mathrm{mmol})$ and $\mathrm{H} 2$ (2 g, $8.9 \mathrm{mmol})$ in the presence of potassium carbonate $(0.89 \mathrm{~g}$, $8.9 \mathrm{mmol})$, to yield $0.95 \mathrm{~g}(30 \%)$ of 77 as light yellow crystals. Melting point $192^{\circ} \mathrm{C}-193^{\circ} \mathrm{C}$; IR 3,383, 3,350, 3,093, 2,240, 1,683, 1,666, 1,323 $\mathrm{cm}^{-1}$; ${ }^{1} \mathrm{H}$ NMR (400 mHz, DMSO-d6): $\delta 4.85\left(\mathrm{~d}, 2 \mathrm{H}, \mathrm{CH}_{2}, \mathrm{~J}=5.1 \mathrm{~Hz}\right), 6.59(\mathrm{t}, 1 \mathrm{H}, \mathrm{NH}-\mathrm{Ar}, \mathrm{J}=4.1 \mathrm{~Hz})$, $6.77(\mathrm{~d}, 2 \mathrm{H}, \mathrm{Ar}-\mathrm{H}, \mathrm{J}=8.3 \mathrm{~Hz}), 7.04$ (t, 1H, Ar-H, J=7.3 Hz), 7.31 (t, 2H, Ar-H, J=7.1 Hz), 7.75 (m, 4H, Ar-H), 8.07 (d, 2H, Ar-H, J=7 Hz), 8.23 (d, 2H, Ar-H, J=7.8 Hz), 9.81 (s, 1H, CO-NH); ${ }^{13} \mathrm{C}-\mathrm{NMR}$ (DMSO-d6): $\delta 50.05,111.35,115.2$, $120.04,120.23,123.5,128.32,128.48,129.02,132.39$, 132.70, 139.9, 136.5, 150.90, 165.69, 195.50; MS (EI) m/z: $355(\mathrm{M}+, 100 \%)$.

\section{4-[2-(2-methoxyphenyl)-2-oxoethylamino]-} $\mathrm{N}$-phenylbenzamide (78)

Prepared by reacting $23(1.85 \mathrm{~g}, 8.7 \mathrm{mmol})$ and $\mathrm{H} 3$ (2 g, $8.7 \mathrm{mmol})$ in the presence of potassium carbonate $(0.87 \mathrm{~g}$,
$8.7 \mathrm{mmol})$, to yield $1.5 \mathrm{~g}(56 \%)$ of 78 as yellow crystals. Melting point $171^{\circ} \mathrm{C}-175^{\circ} \mathrm{C}$; IR 3,383, 3,317, 3,051, 1,666, 1,637, 1,317, 1,026 cm $\mathrm{cm}^{-1}$; $\mathrm{H}$ NMR (400 mHz, DMSO-d6): $\delta 3.96\left(\mathrm{~s}, 3 \mathrm{H}, \mathrm{CH}_{3}\right), 4.57\left(\mathrm{~d}, 2 \mathrm{H}, \mathrm{CH}_{2}, \mathrm{~J}=5.6 \mathrm{~Hz}\right), 6.6(\mathrm{t}, 1 \mathrm{H}$, NH-Ar, J=5.4 Hz), 6.66 (d, 2H, Ar-H, J=8.6 Hz), 7.03 (t, 1H, Ar-H, J=7.1 Hz), 7.07 (t, 1H, Ar-H, J=7.3 Hz), 7.22 (d, 1H, Ar-H, J=8.3 Hz), 7.3 (t, 2H, Ar-H, J=7.8 Hz), 7.59 (t, 2H, Ar-H, J=7.7 Hz), 7.68 (d, 2H, Ar-H, J=7.6 Hz), 7.77 (m, 3H, Ar-H), 9.80 (s, 1H, CO-NH); ${ }^{13} \mathrm{C}-\mathrm{NMR}$ (DMSOd6): $\delta 53.37,55.92,111.20,112.52,120.10,120.62$, $121.66,122.88,125.77,128.40,129.17,129.63,134.23$, $139.71,151.18,158.68,165.25,197.78 ; \mathrm{MS}(\mathrm{EI}) \mathrm{m} / \mathrm{z}: 361$ $(\mathrm{M}+, 100 \%)$.

\section{4-[2-(3-bromophenyl)-2-oxoethylamino]-} N-phenylbenzamide (79)

Prepared by reacting 23 (1.59 g, $7.5 \mathrm{mmol})$ and $\mathrm{H} 4$ ( $2 \mathrm{~g}, 7.5 \mathrm{mmol})$ in the presence of potassium carbonate (0.75 g, $7.5 \mathrm{mmol})$, to yield $0.9 \mathrm{~g}(40 \%)$ of 79 as light yellow crystals. Melting point $200^{\circ} \mathrm{C}-203^{\circ} \mathrm{C}$; IR 3,375, $3,319,3,051,1,687,1,643,1,319,1,186 \mathrm{~cm}^{-1} ;{ }^{1} \mathrm{H}$ NMR (400 mHz, DMSO-d6): $\delta 4.75$ (d, 2H, $\mathrm{CH}_{2}, \mathrm{~J}=8.5 \mathrm{~Hz}$ ), 6.61 (t, 1H, NH-Ar, J=8.8 Hz), 6.78 (d, 2H, Ar-H, J=8 Hz), 7.03 (t, 1H, Ar-H, J=6.9 Hz), $7.3(\mathrm{t}, 2 \mathrm{H}, \mathrm{Ar}-\mathrm{H}, \mathrm{J}=7.5 \mathrm{~Hz}$ ), 7.52 (t, 1H, Ar-H, J=7.9 Hz), 7.76(d, 2H, Ar-H, J=8.5 Hz), 7.81 (d, 2H, Ar-H, J=8 Hz), 7.87 (d, 1H, Ar-H, J=7.6 Hz), 8.07 (d, 1H, Ar-H, J=7.2 Hz), 8.24 (s, 1H, Ar-H), 9.85 (s, 1H, NH-CO); ${ }^{13} \mathrm{C}-\mathrm{NMR}$ (DMSO-d6): $\delta$ 52.35, 114.16, 122.5, 124.49 , 124.91, 125.61, 129.57, 130.5, 131.82, 133.19, 133.66, 138.84, 139.69, 142.37, 153.72, 167.95, 197.95; MS (EI) m/z: 399 (M+, 100\%).

\section{4-[2-(4-fluorophenyl)-2-oxoethylamino]-}

N-phenylbenzamide (80)

Prepared by reacting 23 (1.96 g, $9.2 \mathrm{mmol})$ and $\mathrm{H} 5$ (2 g, $9.2 \mathrm{mmol})$ in the presence of potassium carbonate $(0.92 \mathrm{~g}$, $9.2 \mathrm{mmol})$, to yield $1.9 \mathrm{~g}(59 \%)$ of 80 as white crystals. Melting point $222^{\circ} \mathrm{C}-224^{\circ} \mathrm{C}$; IR 3,385, 3,072, 1,680, 1,643, 1,319, 1,193 $\mathrm{cm}^{-1} ;{ }^{1} \mathrm{H}$ NMR (400 mHz, DMSO-d6): $\delta 4.78$ (d, 2H, $\mathrm{CH}_{2}, \mathrm{~J}=4 \mathrm{~Hz}$ ), 6.54 (t, 1H, NH-Ar, J=4 Hz), 6.78 (d, $2 \mathrm{H}, \mathrm{Ar}-\mathrm{H}, \mathrm{J}=8 \mathrm{~Hz}$ ), 7.03 (t, 1H, Ar-H, J=8 Hz), 7.30 (t, 2H, Ar-H, J=8 Hz), 7.38 (t, 2H, Ar-H, J=8 Hz), 7.77 (d, 2H, Ar-H, $\mathrm{J}=8 \mathrm{~Hz}), 7.81$ (d, 2H, Ar-H, J=8 Hz), 8.17 (t, 2H, Ar-H, J=8 Hz), 9.83 (s, $1 \mathrm{H}, \mathrm{CO}-\mathrm{NH}) ;{ }^{13} \mathrm{C}-\mathrm{NMR}$ (DMSO-d6): $\delta 52.12,114.13$, $118.37,118.58,122.83,124.46,125.60,131.10,131.84$, $133.58,133.67,134.41,142.38,153.76,166.67,167.95$, 169.17, 197.45; MS (EI) m/z: 349 (M+, 100\%). 


\section{4-[2-(3-nitrophenyl)-2-oxoethylamino]- $\mathrm{N}$-phenylbenzamide (8I)}

Prepared by reacting 23 (2.61 g, $12.3 \mathrm{mmol})$ and $\mathrm{H} 6$ ( $3 \mathrm{~g}, 12.3 \mathrm{mmol})$ in the presence of potassium carbonate $(0.62 \mathrm{~g}, 12.3 \mathrm{mmol})$, to yield $1.3 \mathrm{~g}(27 \%)$ of 81 as bright yellow crystals. Melting point $201^{\circ} \mathrm{C}-203^{\circ} \mathrm{C}$; IR 3,394 , $3,072,1,687,1,645,1,531,1,350,1,315 \mathrm{~cm}^{-1} ;{ }^{1} \mathrm{H}$ NMR (400 mHz, DMSO-d6): $\delta 4.9\left(\mathrm{~d}, 2 \mathrm{H}, \mathrm{CH}_{2}, \mathrm{~J}=3.6 \mathrm{~Hz}\right.$ ), 6.61 (s, 1H, NH-Ar), 6.78 (d, 2H, Ar-H, J=8.4 Hz), 7.04 (t, 1H, Ar-H, J=7.3 Hz), 7.3 (t, 2H, Ar-H, J=7.5 Hz), 7.73 (d, 2H, Ar-H, J=8.1 Hz), 7.77 (d, 2H, Ar-H, J=8.5 Hz), 7.87 (t, 1H, Ar-H, J=8 Hz), 8.51 (d, 2H, Ar-H, J=7.8 Hz), 8.76 (s, 1H, Ar-H), 9.82 (s, 1H, CO-NH); ${ }^{13} \mathrm{C}-\mathrm{NMR}$ (DMSO-d6):

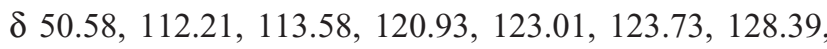
$129.14,129.84,130.00,131.31,134.87,140.29,148.77$, 151.73, 166.01, 195.83; MS (EI) m/z: 376 (M+, 100\%).

\section{4-[2-(4-bromophenyl)-2-oxoethylamino]- $\mathrm{N}$-phenylbenzamide (82)}

Prepared by reacting 23 (3 g, $14.1 \mathrm{mmol})$ and $\mathrm{H} 7(3.93 \mathrm{~g}$, $14.1 \mathrm{mmol})$ in the presence of potassium carbonate $(1.98 \mathrm{~g}$, $14.1 \mathrm{mmol})$, to yield $1.85 \mathrm{~g} \mathrm{(32 \% )}$ of 82 as white crystals. Melting point $225^{\circ} \mathrm{C}-226^{\circ} \mathrm{C}$; IR 3,390, 3,059, 1,678, $1,643,1,319 \mathrm{~cm}^{-1} ;{ }^{1} \mathrm{H}$ NMR (400 mHz, DMSO-d6): $\delta 4.77$ $\left(\mathrm{d}, 2 \mathrm{H}, \mathrm{CH}_{2}, \mathrm{~J}=5 \mathrm{~Hz}\right) ; 6.55$ (t, 1H, NH-Ar, J=5 Hz); 6.74 (d, 2H, Ar-H, J=8.4 Hz); 7.05 (t, 1H, Ar-H, J=7.1 Hz); 7.3 (t, 2H, Ar-H, J=7.7 Hz); 7.73 (d, 2H, Ar-H, J=8.1 Hz); 7.77 (d, 2H, Ar-H, J=7.1 Hz); 7.78 (d, 2H, Ar-H, J=8.4 Hz); 8.01 (d, 2H, Ar-H, J=8.2 Hz); 9.82 (s, 1H, CO-NH); ${ }^{13} \mathrm{C}-\mathrm{NMR}$ (DMSO-d6): $\delta 50.06,112.02,120.72,122.30,123.51,128.49$, 129.02, 129.38, 129.73, 134.20, 135.58, 140.27, 151.27, 151.70, 165.84, 196.75; MS (EI) m/z: 409 (M+, 100\%).

\section{4-[2-oxo-2-(4-trifluoromethylphenyl)ethylamino]- $\mathrm{N}$-phenylbenzamide (83)}

Prepared by reacting $23(1.53 \mathrm{~g}, 7.2 \mathrm{mmol})$ and $\mathrm{H} 8(2 \mathrm{~g}$, $7.2 \mathrm{mmol})$ in the presence of potassium carbonate $(0.72 \mathrm{~g}$, $7.2 \mathrm{mmol})$, to yield $1.7 \mathrm{~g} \mathrm{(58 \% )} \mathrm{of} 83$ as light yellow crystals. Melting point $232^{\circ} \mathrm{C}-234^{\circ} \mathrm{C}$; IR 3,388, 3,037, 1,681, 1,643, 1,330, $1,070 \mathrm{~cm}^{-1}$; ${ }^{1} \mathrm{H}$ NMR (400 mHz, DMSO-d6): $\delta 4.86$ (s, 2H, $\mathrm{CH}_{2}$ ), 6.60 (s, 1H, NH-Ar), 6.77 (s, 2H, Ar-H), 7.03 (s, 1H, Ar-H), 7.30 (s, 2H, Ar-H), 7.76 (s, 4H, Ar-H), 7.94 (s, 2H, Ar-H), 8.27 (s, 2H, Ar-H), 9.81 (s, 1H, CO-NH); ${ }^{13} \mathrm{C}-\mathrm{NMR}$ (DMSO-d6): $\delta 50.40,111.99,120.71,120.89,122.41,123.48$, 124.26 (C-F, J=1242 Hz), 126.24 (C-F, J=14.4 Hz), 128.95, 129.29, 129.68, 133.37 (C-F, J=139.12 Hz), 138.75, 140.19, 151.55, 165.80, 196.4194; MS (EI) m/z: 410 (M+, 100\%).

\section{4-[2-(4-chlorophenyl)-2-oxoethylamino]- $\mathrm{N}$-phenylbenzamide (84)}

Prepared by reacting 23 (2.73 g, $15.4 \mathrm{mmol})$ and H9 (3.6 g, $15.4 \mathrm{mmol})$ in the presence of potassium carbonate $(1.29 \mathrm{~g}$, $15.4 \mathrm{mmol})$, to yield $3.6 \mathrm{~g}(78 \%)$ of 84 as white crystals. Melting point $218^{\circ} \mathrm{C}-219^{\circ} \mathrm{C}$; IR 3,390, 3,039, 1,680, 1,643, $1,321 \mathrm{~cm}^{-1} ;{ }^{1} \mathrm{H}$ NMR (400 mHz, DMSO-d6): $\delta 4.79$ (s, 2H, $\left.\mathrm{CH}_{2}\right), 6.55$ (s, 1H, NH), 6.77 (d, 2H, Ar-H, J=8 Hz), 7.05 (t, 1H, Ar-H, J=7.3 Hz), 7.32 (t, 2H, Ar-H, J=7.4 Hz), 7.65 (d, 2H, Ar-H, J=7.8 Hz), 7.61 (d, 2H, Ar-H, J=126.3 Hz), 7.79 (d, 2H, Ar-H, J=8.1 Hz), 8.1 (d, 2H, Ar-H, J=7.8 Hz), 9.84 (s, CO-NH); ${ }^{13} \mathrm{C}-\mathrm{NMR}$ (DMSO-d6): $\delta 52.18,114.12,122.86$, $124.42,125.66,131.12,131.57,131.82,132.50,136.31$, 141.19, 142.30, 153.73, 167.97, 198.01; MS (EI) m/z: 365 $(\mathrm{M}+, 100 \%)$.

\section{N-(4-methoxyphenyl)-4-[2-(4-methoxyphenyl)- 2-oxoethylamino]benzamide (87)}

Prepared by reacting $33(0.5 \mathrm{~g}, 2.1 \mathrm{mmol})$ and $47(0.47 \mathrm{~g}$, $2.1 \mathrm{mmol})$ in the presence of potassium carbonate $(0.21 \mathrm{~g}$, $2.1 \mathrm{mmol})$ and sodium iodide $(0.32 \mathrm{~g}, 2.1 \mathrm{mmol})$, to yield $0.36 \mathrm{~g}(40 \%)$ of 87 as off-white crystals. Melting point $222^{\circ} \mathrm{C}-223^{\circ} \mathrm{C}$; IR $3,354,3,008,1,664,1,643,1,321$, $1,029 \mathrm{~cm}^{-1} ;{ }^{1} \mathrm{H}$ NMR (400 mHz, DMSO-d6): $\delta 3.75(\mathrm{~s}, 3 \mathrm{H}$, $\left.\mathrm{CH}_{3}\right), 3.88\left(\mathrm{~s}, 3 \mathrm{H}, \mathrm{CH}_{3}\right), 4.72\left(\mathrm{~d}, 2 \mathrm{H}, \mathrm{CH}_{2}, \mathrm{~J}=5 \mathrm{~Hz}\right), 6.45$ (t, 1H, NH-Ar, J=4.7 Hz), 6.76 (d, 2H, Ar-H, J=8.6 Hz), 6.9 (d, 2H, Ar-H, J=9 Hz), 7.1 (d, 2H, Ar-H, J=8.8 Hz), 7.65 (d, 2H, Ar-H, J=8.9 Hz), 7.78 (d, 2H, Ar-H, J=8.6 Hz), 8.09 (d, 2H, Ar-H, J=8.8 Hz), 9.4 (s, 1H, CO-NH); ${ }^{13} \mathrm{C}-$ NMR (DMSO-d6): $\delta 49.10,55.11,55.57,111.40,113.58$, $113.98,121.75,121.81,127.90,128.94,130.22,132.76$, 150.94, 155.07, 163.43, 164.86, 194.39; MS (EI) m/z: 391 $(\mathrm{M}+, 100 \%)$.

\section{$\mathrm{N}$-(3-chlorophenyl)-4-[2-(3-nitrophenyl)-2- oxoethylamino]benzamide (88)}

Prepared by reacting $31(0.5 \mathrm{~g}, 2 \mathrm{mmol})$ and $45(0.5 \mathrm{~g}$, $2 \mathrm{mmol})$ in the presence of potassium carbonate $(0.2 \mathrm{~g}$, $2 \mathrm{mmol})$, to yield $0.24 \mathrm{~g} \mathrm{(29 \% )} \mathrm{of} 88$ as bright yellow crystals. Melting point $195^{\circ} \mathrm{C}-196^{\circ} \mathrm{C}$; IR 3,402, 3,381, 3,057, 1,667, 1,666, 1,514, 1,350, 1,087 cm ${ }^{-1}$; ${ }^{1} \mathrm{H}$ NMR $(400 \mathrm{mHz}$, DMSO-d6): $\delta 4.92$ (s, 2H, $\mathrm{CH}_{2}$ ), 6.67 (s, 1H, NH-Ar), 6.8 (d, 2H, Ar-H, J=8.3 Hz), 7.09 (d, 1H, Ar-H, J=7.6 Hz), 7.33 (t, 1H, Ar-H, J=8 Hz), 7.68 (d, 1H, Ar-H, J=8 Hz), 7.78 (d, 2H, Ar-H, J=8.3 Hz), 7.88 (t, 1H, Ar-H, J=7.9 Hz), 7.95 (s, 1H, Ar-H), 8.51 (d, 2H, Ar-H, J=7.6 Hz), 8.77 (s, 1H, Ar-H), 9.96 (s, 1H, CO-NH); ${ }^{13} \mathrm{C}-\mathrm{NMR}$ (DMSO-d6): $\delta 50.40,112.05$, 
$118.84,119.92,121.86,122.87,123.06,128.24,129.77$, $130.65,131.12,133.33,134.74,136.73,141.76,148.60$, 151.81, 165.96, 195.56; MS (EI) m/z: 410 (M+, 100\%).

\section{Synthesis of anilinoketones and anilinoesters from $\alpha$-bromoketones/ esters $(85,86,89-94)$}

Amino-N-phenylbenzamide (one equivalent), potassium carbonate (one equivalent), sodium iodide (one equivalent), and $\alpha$-bromoketone (one equivalent) were dissolved in dimethylformamide $(20 \mathrm{~mL})$ and the reaction mixture was stirred for 8 hours at room temperature. The reaction contents were then poured over water with stirring, and the mixture was left until the product precipitated. The product was then filtered, dried, and recrystallized from ethanol.

\section{4-(2-oxo-2-p-tolylethylamino)-}

$\mathrm{N}$-phenylbenzamide (85)

Prepared by reacting 23 (2 g, $9.5 \mathrm{mmol})$ and 48 (1.6 g, $9.5 \mathrm{mmol})$ in the presence of potassium carbonate $(0.96 \mathrm{~g}$, $9.5 \mathrm{mmol})$ and sodium iodide $(1.42 \mathrm{~g}, 9.5 \mathrm{mmol})$ to yield $1.6 \mathrm{~g}$ $(50 \%)$ of 85 as beige crystals. Melting point $224^{\circ} \mathrm{C}-226^{\circ} \mathrm{C}$; IR 3,385, 3,323, 3,051, 1,678, 1,643, 1,325 $\mathrm{cm}^{-1} ;{ }^{1} \mathrm{H}$ NMR (400 mHz, DMSO-d6): $\delta 2.55$ (s, 3H, $\mathrm{CH}_{3}$ ), 4.85 (s, $2 \mathrm{H}$, $\left.\mathrm{CH}_{2}\right), 6.62$ (s, 1H, NH-Ar), 6.86 (d, 2H, Ar-H, J=7.7 Hz), 7.15 (t, 1H, Ar-H, J=7.1 Hz), 7.41 (t, 2H, Ar-H, J=7.2 Hz), 7.48 (d, 2H, Ar-H, J=7.2 Hz), 7.85 (d, 2H, Ar-H, J=8 Hz), 7.89 (d, 2H, Ar-H, J=8 Hz), 8.09 (d, 2H, Ar-H, J=7.2 Hz), 9.93 (s, $1 \mathrm{H}, \mathrm{CO}-\mathrm{NH}$ ); ${ }^{13} \mathrm{C}-\mathrm{NMR}$ (DMSO-d6): $\delta 23.85,51.96,114.11$, 122.87, 124.34, 125.66, 130.64, 131.11, 131.84, 131.99, 135.12, 142.31, 146.78, 153.77, 168.01, 198.25; MS (EI) $\mathrm{m} / \mathrm{z}: 345(\mathrm{M}+, 100 \%)$.

\section{4-(2-naphthalen-2-yl-2-oxoethylamino)-} $\mathrm{N}$-phenylbenzamide (86)

Prepared by reacting 23 (3 g, $13.4 \mathrm{mmol})$ and $49(2.75 \mathrm{~g}$, $13.4 \mathrm{mmol})$ in the presence of potassium carbonate $(1.96 \mathrm{~g}$, $13.4 \mathrm{mmol})$ and sodium iodide $(2.12 \mathrm{~g}, 13.4 \mathrm{mmol})$ to yield $2.75 \mathrm{~g}(51 \%)$ of 86 as light yellow crystals. Melting point $221^{\circ} \mathrm{C}-223^{\circ} \mathrm{C}$; IR 3,373, 3,057, 1,672, 1,645, $1,315 \mathrm{~cm}^{-1} ;{ }^{1} \mathrm{H}$ NMR (400 mHz, DMSO-d6): $\delta 4.95$ (d, 2H, $\mathrm{CH}_{2}, \mathrm{~J}=5.2 \mathrm{~Hz}$ ), 6.62 (t, 1H, NH-Ar, J=5.1 Hz), 6.82 (d, 2H, Ar-H, J=8.6 Hz), 7.04 (t, 1H, Ar-H, J=7.3 Hz), 7.31 (t, 2H, Ar-H, J=7.8 Hz), 7.76 (d, 2H, Ar-H, J=7.9 Hz), 7.81 (d, 2H, Ar-H, J=8.6 Hz), 8.03 (d, 1H, Ar-H, J=8 Hz), 8.07 (s, 2H, Ar-H), 8.15 (d, 1H, Ar-H, $\mathrm{J}=18.3 \mathrm{~Hz}$ ), 9.83 (s, 1H, CO-NH); ${ }^{13} \mathrm{C}-\mathrm{NMR}$ (DMSO-d6): $\delta 52.22,114.15,122.83,124.42,125.61,126.12,129.71$, $130.40,131.11,131.46,131.85,132.24,132.51,134.85$,
134.95, 137.91, 142.38, 153.83, 167.95, 198.75; MS (EI) m/z: $381(\mathrm{M}+, 100 \%)$.

Phenyl (4-phenylcarbamoylphenylamino)acetate (89) Prepared by reacting $23(1.24 \mathrm{~g}, 5.9 \mathrm{mmol})$ and $50(1 \mathrm{~g}$, $5.9 \mathrm{mmol})$ in the presence of potassium carbonate $(0.59 \mathrm{~g}$, $5.9 \mathrm{mmol})$ and sodium iodide $(0.88 \mathrm{~g}, 5.9 \mathrm{mmol})$, to yield $0.96 \mathrm{~g}$ (47\%) of 89 as white crystals. Melting point $202^{\circ} \mathrm{C}-204^{\circ} \mathrm{C}$; IR 3,394, 3,323, 3,051, 1,749, 1,643, 1,317 $\mathrm{cm}^{-1}$; ${ }^{1} \mathrm{H}$ NMR (400 mHz, DMSO-d6): $\delta 4.31$ (d, 2H, $\mathrm{CH}_{2}, \mathrm{~J}=4 \mathrm{~Hz}$ ), 6.75 (d, 2H, Ar-H, J=8 Hz), 6.82 (t, 1H, NH-Ar, J=4 Hz), 7.04 (t, 1H, Ar-H, J=6 Hz), 7.14 (d, 2H, Ar-H, J=8 Hz), 7.26 (t, 1H, Ar-H, J=8 Hz), 7.31 (t, 2H, Ar-H, J=8 Hz), 7.43 (t, 2H, Ar-H, J=6 Hz), 7.76 (d, 2H, Ar-H, J=8 Hz), 7.84 (d, 2H, $\mathrm{Ar}-\mathrm{H}, \mathrm{J}=8 \mathrm{~Hz}$ ), 9.86 (s, 1H, CO-NH); ${ }^{13} \mathrm{C}-\mathrm{NMR}$ (DMSO-d6): $\delta 47.09,113.92,122.82,124.31,124.99,125.66,128.63$, 131.14, 131.96, 132.25, 142.36, 153.02, 153.62, 167.94, 172.60; MS (EI) m/z: 347 (M+, 100\%).

\section{Naphthalen-2-yl (4-phenylcarbamoylphenylamino) acetate (90)}

Prepared by reacting $23(1.44 \mathrm{~g}, 6.8 \mathrm{mmol})$ and $51(1.5 \mathrm{~g}$, $6.8 \mathrm{mmol})$ in the presence of potassium carbonate $(0.70 \mathrm{~g}$, $6.8 \mathrm{mmol}$ ) and sodium iodide ( $3.02 \mathrm{~g}, 6.8 \mathrm{mmol})$ to yield $1.17 \mathrm{~g}(44 \%)$ of 90 as light brown crystals. Melting point $233^{\circ} \mathrm{C}-234^{\circ} \mathrm{C}$; IR $3,408,3,350,3,047,1,759,1,643$, $1,336 \mathrm{~cm}^{-1} ;{ }^{1} \mathrm{H}$ NMR (400 mHz, DMSO-d6): $\delta 4.38$ (s, 2H, $\mathrm{CH}_{2}$ ), 5.34 (s, 1H, NH-CO), 6.77 (d, 2H, Ar-H, J=8.7 Hz), 7.04 (t, 1H, Ar-H, J=7.3 Hz), 7.31 (m, 3H, Ar-H), 7.52 (m, 2H, Ar-H), 7.68 (s, 1H, Ar-H), 7.74 (d, 2H, Ar-H, J=7.7 Hz), 7.83 (d, 2H, Ar-H, J=8.7 Hz), 7.93 (t, 2H, Ar-H, J=9.2 Hz), 7.98 (d, 1H, Ar-H, J=9 Hz), 9.85 (s, 1H, NH-CO); ${ }^{13} \mathrm{C}-\mathrm{NMR}$ (DMSO-d6): $\delta$ 39.73, 106.54, 113.67, 115.42, 116.64, $117.58,118.28,121.17,122.04,122.74,122.97,123.74$, $124.57,124.72,126.29,128.54,134.93,143.28,146.23$, 160.54, 165.43; MS (EI) m/z: 397 (M+, 100\%).

\section{Naphthalen-2-yl (3-phenylcarbamoylphenylamino) acetate (9I)}

Prepared by reacting $24(1.44 \mathrm{~g}, 6.8 \mathrm{mmol})$ and $51(1.5 \mathrm{~g}$, $6.8 \mathrm{mmol})$ in the presence of potassium carbonate $(0.69 \mathrm{~g}$, $6.8 \mathrm{mmol})$ and sodium iodide $(2.02 \mathrm{~g}, 6.8 \mathrm{mmol})$ to yield $1.6 \mathrm{~g}(60 \%)$ of 91 as off-white crystals. Melting point $169^{\circ} \mathrm{C}-170^{\circ} \mathrm{C}$; IR $3,398,3,317,3,061,1,753,1,651$, $1,321 \mathrm{~cm}^{-1} ;{ }^{1} \mathrm{H}$ NMR (400 mHz, DMSO-d6): $\delta 4.36$ (d, $2 \mathrm{H}, \mathrm{CH}_{2}, \mathrm{~J}=6.2 \mathrm{~Hz}$ ), 6.50 (t, 1H, NH-Ar, J=6.2 Hz), 6.9 (d, 1H, Ar-H, J=7.8 Hz), 7.09 (t, 1H, Ar-H, J=7.9 Hz), 7.21 (d, 1H, Ar-H, J=8.4 Hz), 7.22 (s, 1H, Ar-H), 7.29 (t, 1H, 
Ar-H, J=8.1 Hz), 7.33 (t, 2H, Ar-H, J=7.9 Hz), $7.36(\mathrm{~d}, 1 \mathrm{H}$, Ar-H, J=7.5 Hz), 7.53 (m, 2H, Ar-H), 7.69 (s, 1H, Ar-H), 7.78 (d, 2H, Ar-H, J=8 Hz), 7.9 (d, 1H, Ar-H, J=7.7 Hz), 7.95 (d, 1H, Ar-H, J=7.6 Hz), 7.98 (d, 1H, Ar-H, J=9.2 Hz), 10.13 (s, 1H, CO-NH); ${ }^{13} \mathrm{C}-\mathrm{NMR}$ (DMSO-d6): $\delta 44.79$, $111.31,115.20,115.66,118.38,120.32,121.35,123.48$, $125.85,126.72,127.42,127.67,128.52,128.92,129.92$, $130.99,133.25,135.93,139.25,148.01,148.04,166.11$, 170.44; MS (EI) m/z: $397(\mathrm{M}+, 75 \%)$.

\section{Naphthalen-2-yl [4-(4-chlorophenylcarbamoyl) phenylamino]acetate (92)}

Prepared by reacting $26(1.68 \mathrm{~g}, 6.8 \mathrm{mmol})$ and $51(1.5 \mathrm{~g}$, $6.8 \mathrm{mmol})$ in the presence of potassium carbonate $(0.68 \mathrm{~g}$, $6.8 \mathrm{mmol}$ ) and sodium iodide (1.02 g, $6.8 \mathrm{mmol})$ to yield $2.3 \mathrm{~g}$ (79\%) of 92 as white crystals. Melting point $198^{\circ} \mathrm{C}-200^{\circ} \mathrm{C}$; IR 3,373, 3,062, 1,753, 1,664, 1,323, 1,095 $\mathrm{cm}^{-1}$; ${ }^{1} \mathrm{H}$ NMR (400 mHz, DMSO-d6): $\delta 4.37\left(\mathrm{~s}, 2 \mathrm{H}, \mathrm{CH}_{2}\right), 6.54(\mathrm{~s}, 1 \mathrm{H}$, NH-Ar), 6.92 (d, 1H, Ar-H, J=6.7 Hz), 7.22 (s, 2H, Ar-H), 7.3 (t, 2H, Ar-H, J=8.3 Hz), 7.42 (d, 2H, Ar-H, J=8 Hz), 7.54 (s, 2H, Ar-H), 7.84 (d, 2H, Ar-H, J=7.9 Hz), 7.91 (d, 2H, Ar-H, $\mathrm{J}=7.4 \mathrm{~Hz}$ ), 7.96 (d, 2H, Ar-H, J=6.9 Hz), 10.29 (s, 1H, CO-NH); ${ }^{13} \mathrm{C}-\mathrm{NMR}$ (DMSO-d6): $\delta$ 43.98, 110.53, 114.55, 114.88, $117.57,120.52,121.02,125.04,125.92,126.60,126.86$, $127.64,128.18,128.60,130.18,134.83,137.41,147.20$, 147.26, 159.02, 165.44; MS (EI) m/z: $431(\mathrm{M}+, 100 \%)$.

\section{Naphthalen-2-yl (4-p-tolylcarbamoylphenylamino) acetate (93)}

Prepared by reacting $30(1.54 \mathrm{~g}, 6.8 \mathrm{mmol})$ and $51(1.5 \mathrm{~g}$, $6.8 \mathrm{mmol})$ in the presence of potassium carbonate $(0.68 \mathrm{~g}$, $6.8 \mathrm{mmol})$ and sodium iodide (1.02 $\mathrm{g}, 6.8 \mathrm{mmol})$ to yield $1.7 \mathrm{~g}$ $(61 \%)$ of 93 as white crystals. Melting point $202^{\circ} \mathrm{C}-204^{\circ} \mathrm{C}$; IR 3,396, 3,275, 3,059, 1,751, 1,643, 1,319 $\mathrm{cm}^{-1}$; ${ }^{1} \mathrm{H}$ NMR (400 mHz, DMSO-d6): $\delta 2.27\left(\mathrm{~s}, 3 \mathrm{H}, \mathrm{CH}_{3}\right), 4,36(\mathrm{~s}, 2 \mathrm{H}$, $\mathrm{CH}_{2}$ ), 6.51 (s, 1H, NH-Ar), 6.89 (s, 1H, Ar-H), 7.15 (s, 2H, Ar-H), 7.21 (s, 2H, Ar-H), 7.29 (d, 2H, Ar-H, J=11.1 Hz), 7.53 (s, 2H, Ar-H), 7.65 (s, 3H, Ar-H), 7.93 (m, 3H, Ar-H), 10.07 (s, 1H, CO-NH); ${ }^{13} \mathrm{C}-\mathrm{NMR}$ (DMSO-d6): $\delta 20.98$, $45.35,111.81,115.70,116.19,118.91,120.92,121.87$, $126.38,127.26,127.95,128.20,129.45,129.94,131.52$, $132.97,133.78,136.52,137.24,148.55,166.44,170.98 ; \mathrm{MS}$ (EI) $\mathrm{m} / \mathrm{z}: 411(\mathrm{M}+, 100 \%)$.

\section{Naphthalen-2-yl [4-(3-chlorophenylcarbamoyl) phenylamino]acetate (94)}

Prepared by reacting $32(1.68 \mathrm{~g}, 6.8 \mathrm{mmol})$ and $51(1.5 \mathrm{~g}$, $6.8 \mathrm{mmol})$ in the presence of potassium carbonate $(0.68 \mathrm{~g}$,
$6.8 \mathrm{mmol})$ and sodium iodide (1.02 $\mathrm{g}, 6.8 \mathrm{mmol})$ to yield $2.1 \mathrm{~g}$ $(72 \%)$ of 94 as white crystals. Melting point $154^{\circ} \mathrm{C}-155^{\circ} \mathrm{C}$; IR 3,392, 3,234, 3,068, 1,762, 1,647, 1,336 cm ${ }^{-1}$; ${ }^{1} \mathrm{H}$ NMR (400 mHz, DMSO-d6): $\delta 4.36$ (d, 2H, $\left.\mathrm{CH}_{2}, \mathrm{~J}=5.4 \mathrm{~Hz}\right), 6.53$ (t, 1H, NH-Ar, J=5.4 Hz), 6.92 (d, 1H, Ar-H, J=8.6 Hz), 7.08 (d, 1H, Ar-H, J=8.6 Hz), 7.13 (m, 2H, Ar-H), $7.22(\mathrm{~m}, 2 \mathrm{H}$, Ar-H), 7.3 (t, 1H, Ar-H, J=8.7 Hz), $7.36(t, 1 \mathrm{H}, \mathrm{Ar}-\mathrm{H}$, $\mathrm{J}=13.3 \mathrm{~Hz}), 7.36$ (m, 2H, Ar-H), 7.51 (m, 2H, Ar-H), 7.67 (m, 2H, Ar-H), 7.93 (m, 3H, Ar-H), 10.35 (s, 1H, NH-CO); ${ }^{13}$ C-NMR (DMSO-d6): $\delta$ 40.05, 106.60, 110.76, 110.98, $113.69,113.86,114.93,116.64,118.46,121.15,122.02$, $122.71,122.97,124.31,124.71,125.55,128.20,128.54$, 130.80, 136.04, 143.38, 161.71, 165.72; MS (EI) m/z: 431 $(\mathrm{M}+, 100 \%)$.

\section{Synthesis of anilinoalcohols (95-120)}

One equivalent of anilinoketone was dissolved in $20 \mathrm{~mL}$ of methanol, to which a 1.2 equivalent of sodium borohydride was added. The round bottom flask was fitted with a bent drying tube filled with silica gel (blue). The reaction was stirred at room temperature for 2 hours. The methanol was then evaporated under vacuum and the residue was dissolved in $50 \mathrm{~mL}$ of dichloromethane and washed with $30 \mathrm{~mL}$ of water, then with $30 \mathrm{~mL}$ of $1 \mathrm{M} \mathrm{HCl}$ solution, and again with $30 \mathrm{~mL}$ of water. The dichloromethane layer was then dried using magnesium sulfate filtered and evaporated under vacuum. The product was recrystallized from ethyl acetate/hexane.

\section{4-(2-hydroxy-2-phenylethylamino)- $\mathrm{N}$-phenylbenzamide (95)}

Prepared by reducing $52(0.5 \mathrm{~g}, 1.5 \mathrm{mmol})$ using sodium borohydride $(0.026 \mathrm{~g}, 1.8 \mathrm{mmol})$, to yield $0.24 \mathrm{~g}(48 \%)$ of 95 as yellow crystals. Melting point $150^{\circ} \mathrm{C}-152^{\circ} \mathrm{C}$; IR 3,394, 3,369, 3,348, 3,032, 1,606, 1,321 cm $\mathrm{cm}^{-1}$; ${ }^{1} \mathrm{H}$ NMR (400 mHz, DMSO-d6): $\delta 3.21\left(\mathrm{~m}, 1 \mathrm{H}, \mathrm{CH}_{2}-\mathrm{N}-\mathrm{Ar}\right), 3.28\left(\mathrm{~m}, 1 \mathrm{H}, \mathrm{CH}_{2}-\right.$ N-Ar), 4.76 (d,1H, CH-O, J=3.3 Hz), 5.56 (s, 1H, OH), 6.3 (t, 1H, NH-Ar, J=5.1 Hz), 6.68 (d, 2H, Ar-H, J=8.4 Hz), 7.04 (t, 1H, Ar-H, J=7 Hz), 7.31 (m, 5H, Ar-H), 7.42 (d, 2H, Ar-H, $\mathrm{J}=7.6 \mathrm{~Hz}$ ), 7.75 (t, 4H, Ar-H, J=9.5 Hz), 9.79 (s, 1H, NH-CO);

${ }^{13} \mathrm{C}-\mathrm{NMR}$ (DMSO-d6): $\delta 46.09,66.07,106.26,115.40,116.38$, $118.14,121.32,122.31,123.31,123.69,124.53,135.04$, 139.21, 146.86, 160.52; MS (EI) m/z: 333 (M+, 100\%).

\section{4-(2-hydroxy-2-phenylethylamino)-}

N-p-tolylbenzamide (96)

Prepared by reducing $54(0.5 \mathrm{~g}, 1.5 \mathrm{mmol})$ using sodium borohydride $(0.07 \mathrm{~g}, 1.8 \mathrm{mmol})$ to yield $0.35 \mathrm{~g}(70 \%)$ of 96 as white crystals. Melting point $132^{\circ} \mathrm{C}-133^{\circ} \mathrm{C}$; IR 3,381, 3,367, 
3,037, 1,614, 1,346 cm ${ }^{-1}$; ${ }^{1} \mathrm{H}$ NMR (400 mHz, DMSO-d6): $\delta 2.27\left(\mathrm{~s}, 3 \mathrm{H}, \mathrm{CH}_{3}\right), 3.21\left(\mathrm{~m}, 1 \mathrm{H}, \mathrm{CH}_{2}-\mathrm{N}-\mathrm{Ar}\right), 3.29(\mathrm{~m}, 1 \mathrm{H}$, $\left.\mathrm{CH}_{2}-\mathrm{N}-\mathrm{Ar}\right), 4.76$ (d, 1H, CH-O, J=3.3 Hz), 5.55 (d, 1H, OH, $\mathrm{J}=3.8 \mathrm{~Hz}$ ), 6.32 (s, 1H, NH-Ar), 6.69 (d, 2H, Ar-H, J=8.4 Hz), 7.11 (d, 2H, Ar-H, J=8 Hz), 7.27 (t, 1H, Ar-H, J=7 Hz), 7.36 (t, 2H, Ar-H, J=7.3 Hz), 7.63 (d, 2H, Ar-H, J=8 Hz), 7.76 (d, 2H, Ar-H, J=8.3 Hz), 9.72 (s, 1H, NH-CO); ${ }^{13} \mathrm{C}-\mathrm{NMR}$ (DMSO-d6): $\delta$ 15.70, 46.08, 66.04, 106.24, 115.44, 116.46, $121.30,122.30,123.30,124.09,124.43,127.02,132.45$, 139.19, 146.74, 160.34; MS (EI) m/z: 348 (M+, 100\%).

\section{N-(4-chlorophenyl)-4-(2-hydroxy-2-}

phenylethylamino)benzamide (97)

Prepared by reducing 58 ( $0.5 \mathrm{~g}, 1.4 \mathrm{mmol})$ using sodium borohydride $(0.062 \mathrm{~g}, 1.68 \mathrm{mmol})$, to yield $0.34 \mathrm{~g}(68 \%)$ of 97 as white crystals. Melting point $169^{\circ} \mathrm{C}-171^{\circ} \mathrm{C}$; IR 3,562, 3,410, 3,385, 3,030, 1,643, 1,328, 1,087 $\mathrm{cm}^{-1}$; ${ }^{1} \mathrm{H}$ NMR (400 mHz, DMSO-d6): $\delta 3.21\left(\mathrm{~m}, 1 \mathrm{H}, \mathrm{CH}_{2}-\mathrm{N}-\mathrm{Ar}\right), 3.31\left(\mathrm{~m}, 1 \mathrm{H}, \mathrm{CH}_{2}-\right.$ $\mathrm{N}-\mathrm{Ar}), 4.77$ (t, 1H, CH-O, J=3.4 Hz), 5.57 (d, 1H, OH, $\mathrm{J}=4.2 \mathrm{~Hz}$ ), 6.39 (t, 1H, NH-Ar, J=5.3 Hz), 6.7 (d, 2H, Ar-H, $\mathrm{J}=8.5 \mathrm{~Hz}), 7.27$ (t, 1H, Ar-H, J=7.1 Hz), 7.36 (m, 4H, Ar-H), 7.55 (d, 2H, Ar-H, J=7.8 Hz), 7.78 (d, 2H, Ar-H, J=8.6 Hz), 7.81 (d, 2H, Ar-H, J=8.8 Hz), 9.93 (s, 1H, CO-NH); ${ }^{13} \mathrm{C}-\mathrm{NMR}$ (DMSO-d6): $\delta 49.93,69.95,110.15,119.86,120.73,125.20$, 125.60, 126.21, 127.20, 127.64, 128.49, 137.90, 143.06, 150.89, 164.49; MS (EI) m/z: 367 (M+, 100\%).

\section{N-(3-chlorophenyl)-4-(2-hydroxy-2-}

\section{phenylethylamino)benzamide (98)}

Prepared by reducing $60(0.5 \mathrm{~g}, 1.4 \mathrm{mmol})$ using sodium borohydride $(0.062 \mathrm{~g}, 1.68 \mathrm{mmol})$ to yield $0.2 \mathrm{~g}(40 \%)$ of 98 as white crystals. Melting point $181^{\circ} \mathrm{C}-182^{\circ} \mathrm{C}$; IR 3,410, 3,338, 3,240, 3,028, 1,651, 1,311, 1,089 $\mathrm{cm}^{-1} ;{ }^{1} \mathrm{H}$ NMR (400 mHz, DMSO-d6): $\delta 3.2\left(\mathrm{~m}, 1 \mathrm{H}, \mathrm{CH}_{2}-\mathrm{N}-\mathrm{Ar}\right), 3.29$ (m, 1H, $\left.\mathrm{CH}_{2}-\mathrm{N}-\mathrm{Ar}\right), 4.78$ (s, 1H, CH-O), 5.54 (s, 1H, OH), $5.88(\mathrm{t}, 1 \mathrm{H}, \mathrm{NH}-\mathrm{Ar}, \mathrm{J}=5.2 \mathrm{~Hz}), 6.86(\mathrm{~d}, 1 \mathrm{H}, \mathrm{Ar}-\mathrm{H}, \mathrm{J}=7.9 \mathrm{~Hz})$, $7.12(\mathrm{~m}, 2 \mathrm{H}, \mathrm{Ar}-\mathrm{H}), 7.22$ (t, 1H, Ar-H, J=7.8 Hz), 7.26 (t, 2H, Ar-H, J=7.4 Hz), 7.36 (t, 2H, Ar-H, J=7.4 Hz), $7.41(\mathrm{~m}, 4 \mathrm{H}$, Ar-H), 7.81 (d, 2H, Ar-H, J=8.8 Hz), 10.22 (s, 1H, CO-NH); ${ }^{13} \mathrm{C}-\mathrm{NMR}$ (DMSO-d6): $\delta$ 51.28, 70.83, 110.99, 114.92 , $115.56,121.88,126.09,127.07,127.12,128.09,128.50$, 128.93, 135.60, 138.29, 144.29, 148.78, 166.46; MS (EI) $\mathrm{m} / \mathrm{z}: 367(\mathrm{M}+, 100 \%)$.

\section{4-(2-hydroxy-2-phenylethylamino)-}

\section{$\mathrm{N}$-(4-methoxyphenyl)benzamide (99)}

Prepared by reducing $62(0.5 \mathrm{~g}, 1.4 \mathrm{mmol})$ using sodium borohydride $(0.06 \mathrm{~g}, 1.68 \mathrm{mmol})$ to yield $0.32 \mathrm{~g}(64 \%)$ of
99 as white crystals. Melting point $165^{\circ} \mathrm{C}-166^{\circ} \mathrm{C}$; IR 3,408, 3,361, 3,331, 3,043, 1,612, 1,313, 1,246, 1,031 cm ${ }^{-1} ;{ }^{1} \mathrm{H}$ NMR (400 mHz, DMSO-d6): $\delta 3.22\left(\mathrm{~m}, 1 \mathrm{H}, \mathrm{CH}_{2}-\mathrm{N}-\mathrm{Ar}\right), 3.30$ (m, 1H, $\left.\mathrm{CH}_{2}-\mathrm{N}-\mathrm{Ar}\right), 3.18$ (s, 3H, $\mathrm{CH}_{3}$ ), 4.75 (m, 1H, CH-O), 5.57 (d, 1H, OH, J=4.3 Hz), 6.38 (t, 1H, NH-Ar, J=5.5 Hz), $6.68(\mathrm{~d}, 2 \mathrm{H}, \mathrm{Ar}-\mathrm{H}, \mathrm{J}=8.6 \mathrm{~Hz}), 6.9$ (d, 2H, Ar-H, J=8.9 Hz), 7.27 (t, 1H, Ar-H, J=7.5 Hz), 7.36 (t, 2H, Ar-H, J=7.5 Hz), 7.42 (d, 2H, Ar-H, J=7.4 Hz), 7.64 (d, 2H, Ar-H, J=8.9 Hz), 7.76 (d, $2 \mathrm{H}, \mathrm{Ar}-\mathrm{H}, \mathrm{J}=8.6 \mathrm{~Hz}$ ), 9.70 (s, 1H, CO-NH); ${ }^{13} \mathrm{C}-\mathrm{NMR}$ (DMSOd6): $\delta 51.31,55.65,71.30,111.50,114.12,121.70,122.34$, $126.55,127.58,128.57,129.62,133.25,144.40,151.93$, 155.61, 165.48; MS (EI) m/z: 363 (M+, 100\%).

\section{4-(2-hydroxy-2-phenylethylamino)-}

\section{$\mathrm{N}$-(3-methoxyphenyl)benzamide (100)}

Prepared by reducing 64 (0.5 g, $1.4 \mathrm{mmol})$ using sodium borohydride $(0.063 \mathrm{~g}, 1.68 \mathrm{mmol})$ to yield $0.28 \mathrm{~g}(56 \%)$ of 100 as yellow oil. It was purified using preparative thin layer chromatography before characterization. IR 3,408, 3,360, 3,000, 1,651, 1,325, 1,039 $\mathrm{cm}^{-1} ;{ }^{1} \mathrm{H} \mathrm{NMR}(400 \mathrm{mHz}$, DMSO-d6): $\delta 3.21\left(\mathrm{~s}, 1 \mathrm{H}, \mathrm{CH}_{2}-\mathrm{N}-\mathrm{Ar}\right), 3.32\left(\mathrm{~s}, 1 \mathrm{H}, \mathrm{CH}_{2}-\right.$ N-Ar), 3.73 (s, 3H, $\left.\mathrm{CH}_{3}\right), 4.75$ (s, 1H, CH-O), 5.53 (s, 1H, $\mathrm{OH}), 6.32$ (s, 1H, NH-Ar), 6.61 (s, 1H, Ar-H), 6.68 (s, 2H, Ar-H), 7.19 (s, 1H, Ar-H), 7.26 (s, 1H, Ar-H), 7.34 (s, 3H, Ar-H), 7.41 (s, 2H, Ar-H), 7.46 (s, 1H, Ar-H), 7.75 (s, 2H, Ar-H), 9.97 (s, 1H, CO-NH); ${ }^{13}$ C-NMR (DMSO-d6): $\delta 51.28$, 55.43, 71.26, 106.22, 108.91, 111.46, 112.82, 121.55, 126.55, 127.56, 128.55, 129.69, 129.76, 141.17, 144.82, 152.14, 159.85, 165.77; MS (EI) m/z: 363 (M+, 97\%).

\section{$\mathrm{N}$-[3-(I-hydroxyethyl)phenyl]-4-(2-hydroxy-}

2-phenylethylamino)benzamide ( $\mathrm{IO}$ I)

Prepared by reducing 68 (0.5 g, $1.6 \mathrm{mmol})$ using sodium borohydride $(0.144 \mathrm{~g}, 3.84 \mathrm{mmol})$, to yield $0.39 \mathrm{~g}(78 \%)$ of 101 as light yellow oil. It was purified using preparative thin layer chromatography before characterization. IR 3,344, 2,954, 1,626, 1,319, 1,083 cm ${ }^{-1}$; ${ }^{1} \mathrm{H}$ NMR (400 mHz, DMSO-d6): $\delta 1.36$ (s, 3H, $\left.\mathrm{CH}_{3}\right), 3.24\left(\mathrm{~m}, 1 \mathrm{H}, \mathrm{CH}_{2}-\mathrm{N}-\mathrm{Ar}\right)$, 3.31 (m, 1H, $\left.\mathrm{CH}_{2}-\mathrm{N}-\mathrm{Ar}\right), 4.71$ (s, 1H, CH-O), 4.79 (s, 1H, CH-O), 5.19 (s, 1H, OH), 5.59 (s, 1H, OH), 6.35 (s, 1H, NH), 6.71 (d, 1H, Ar-H, J=8.4 Hz), 7.04 (d, 2H, Ar-H, J=7 Hz), 7.26 (m, 2H, Ar-H), 7.37 (t, 2H, Ar-H, J=7.4 Hz), 7.45 (d, 2H, Ar-H, J=7.4 Hz), 7.68 (d, 1H, Ar-H, J=7.6 Hz), 7.77 (s, 1H, Ar-H), 7.82 (d, 2H, Ar-H, J=8.4 Hz), 9.80 (s, 1H, CO-NH); ${ }^{13} \mathrm{C}-\mathrm{NMR}$ (DMSO-d6): $\delta 21.20,46.12,63.47,66.07,106.25$, $112.59,113.75,115.31,116.43,121.33,122.31,123.27$, $123.31,124.52,134.80,139.21,143.03,146.83,160.45 ; \mathrm{MS}$ (EI) $\mathrm{m} / \mathrm{z}: 377(\mathrm{M}+, 100 \%)$. 


\section{Ethyl 3-[4-(2-hydroxy-2-phenylethylamino)} benzoylamino]benzoate (102)

Prepared by reducing $70(0.5 \mathrm{~g}, 1.2 \mathrm{mmol})$ using sodium borohydride $(0.056 \mathrm{~g}, 1.44 \mathrm{mmol})$ to yield $0.29 \mathrm{~g}(58 \%)$ of 102 as white crystals. Melting point $163^{\circ} \mathrm{C}-165^{\circ} \mathrm{C}$; IR 3,456, 3,396, 3,350, 2,937, 1,681, 1,319 $\mathrm{cm}^{-1}$; ${ }^{1} \mathrm{H}$ NMR $(400 \mathrm{mHz}$, DMSO-d6): $\delta 1.32\left(\mathrm{t}, 3 \mathrm{H}, \mathrm{CH}_{3}, \mathrm{~J}=6.8 \mathrm{~Hz}\right), 3.22\left(\mathrm{~m}, 1 \mathrm{H}, \mathrm{CH}_{2}-\right.$ $\mathrm{N}-\mathrm{Ar}$ ), 3.30 (m, 1H, $\left.\mathrm{CH}_{2}-\mathrm{N}-\mathrm{Ar}\right), 4.29$ (q, 2H, $\mathrm{CH}_{2}, \mathrm{~J}=6.9 \mathrm{~Hz}$ ), 4.77 (s, 1H, CH-O), 5.57 (s, 1H, OH), 6.44 (s, 1H, NH-Ar), 6.71 (d, 2H, Ar-H, J=8 Hz), 7.27 (t, 1H, Ar-H, J=6.9 Hz), 7.35 (t, 2H, Ar-H, J=7 Hz), 7.43 (d, 2H, Ar-H, J=7.1 Hz), 7.8 (d, 2H, Ar-H, J=8 Hz), 7.93 (s, 4H, Ar-H), 10.12 (s, 1H, CO-NH); ${ }^{13} \mathrm{C}-\mathrm{NMR}$ (DMSO-d6): $\delta 14.74,60.85,71.31$, $111.52,119.71,120.96,121.00,124.28,126.57,127.57$, 128.56, 130.04, 130.47, 144.42, 144.85, 152.44, 165.96, 166.09; MS (EI) m/z: 405 (M+, 100\%).

\section{3-(2-hydroxy-2-phenylethylamino)- $\mathrm{N}$-phenylbenzamide (103)}

Prepared by reducing $53(0.5 \mathrm{~g}, 1.5 \mathrm{mmol})$ using sodium borohydride $(0.069 \mathrm{~g}, 1.8 \mathrm{mmol})$ to yield $0.34 \mathrm{~g}(68 \%)$ of 103 as beige crystals. Melting point $142^{\circ} \mathrm{C}-143^{\circ} \mathrm{C}$; IR 3,514, 3,352, 3,336, 3,032, 1,651, 1,327 $\mathrm{cm}^{-1} ;{ }^{1} \mathrm{H}$ NMR $(400 \mathrm{mHz}$, DMSO-d6): $\delta 3.19$ (m, 1H, $\left.\mathrm{CH}_{2}-\mathrm{N}-\mathrm{Ar}\right), 3.29\left(\mathrm{~m}, 1 \mathrm{H}, \mathrm{CH}_{2}-\right.$ N-Ar), 4.80 (m, 1H, CH-O), 5.54 (d, 1H, OH, J=4.4 Hz), $5.83(\mathrm{t}, 1 \mathrm{H}, \mathrm{NH}-\mathrm{Ar}, \mathrm{J}=5.2 \mathrm{~Hz}), 6.48(\mathrm{~d}, 1 \mathrm{H}, \mathrm{Ar}-\mathrm{H}, \mathrm{J}=7.5 \mathrm{~Hz})$, 7.09 (t, 1H, Ar-H, J=7.4 Hz), 7.13 (m, 2H, Ar-H), 7.21 (t, 1H, Ar-H, J=7.9 Hz), 7.26 (t, 1H, Ar-H, J=7.4 Hz), 7.34 (m, 4H, Ar-H), 7.43 (d, 2H, Ar-H, J=7.2 Hz), 7.75 (d, 2H, Ar-H, $\mathrm{J}=7.6 \mathrm{~Hz}), 10.08$ (s, 1H, CO-NH); ${ }^{13} \mathrm{C}-\mathrm{NMR}$ (DMSO-d6): $\delta 51.75,71.30,111.49,115.43,115.92,120.90,124.04$, $126.55,127.54,128.56,129.06,129.37,136.34,139.73$, 144.53, 149.18, 166.83; MS (EI) m/z: 333 (M+, 100\%).

\section{3-(2-hydroxy-2-phenylethylamino)- \\ N-p-tolylbenzamide ( 104)}

Prepared by reducing $55(0.5 \mathrm{~g}, 1.5 \mathrm{mmol})$ using sodium borohydride $(0.066 \mathrm{~g}, 1.8 \mathrm{mmol})$ to yield $0.3 \mathrm{~g}(60 \%)$ of 104 as beige crystals. Melting point $143^{\circ} \mathrm{C}-144^{\circ} \mathrm{C}$; IR $3,348,3,332,3,030,1,651,1,319 \mathrm{~cm}^{-1}$; ${ }^{1} \mathrm{H}$ NMR $(400 \mathrm{mHz}$, DMSO-d6): $\delta 2.30\left(\mathrm{~s}, 3 \mathrm{H}, \mathrm{CH}_{3}\right), 3.21\left(\mathrm{~m}, 1 \mathrm{H}, \mathrm{CH}_{2}-\mathrm{N}-\mathrm{Ar}\right.$ ), $3.30\left(\mathrm{~m}, 1 \mathrm{H}, \mathrm{CH}_{2}-\mathrm{N}-\mathrm{Ar}\right), 4.79$ (s, 2H, CH-O), 5.56 (s, 1H, $\mathrm{OH}), 5.81$ (s, 1H, NH-Ar), 6.85 (d, 1H, Ar-H, J=7.7 Hz), 7.15 (m, 4H, Ar-H), 7.23 (t, 1H, Ar-H, J=7.8 Hz), 7.29 (t, 1H, Ar-H, J=7 Hz), 7.38 (t, 2H, Ar-H, J=7.4 Hz), 7.45 (d, $2 \mathrm{H}, \mathrm{Ar}-\mathrm{H}, \mathrm{J}=7.5 \mathrm{~Hz}$ ), 7.66 (d, 2H, Ar-H, J=8.2 Hz), 10.02 (s, 1H, NH-CO); ${ }^{13} \mathrm{C}-\mathrm{NMR}$ (DMSO-d6): $\delta$ 20.96, 51.73, 71.29, 111.41, 115.37, 115.81, 120.91, 126.53, 127.52,
128.54, 129.32, 129.43, 132.97, 136.38, 137.18, 144.52, 149.16, 166.61; MS (EI) m/z: 348 (M+, 100\%).

\section{3-(2-hydroxy-2-phenylethylamino)-} $\mathrm{N}$-m-tolylbenzamide ( I05)

Prepared by reducing $57(0.5 \mathrm{~g}, 1.5 \mathrm{mmol})$ using sodium borohydride $(0.07 \mathrm{~g}, 1.8 \mathrm{mmol})$ to yield $0.38 \mathrm{~g}(76 \%)$ of 105 as yellow oil. This was purified using preparative thin layer chromatography before characterization. IR 3,296, $3,028,2,918,1,651,1,311 \mathrm{~cm}^{-1}$; ${ }^{1} \mathrm{H}$ NMR (400 mHz, DMSOd6): $\delta 2.08$ (s, $3 \mathrm{H}, \mathrm{CH}_{3}$ ), 3.19 (s, $\left.1 \mathrm{H}, \mathrm{CH}_{2}-\mathrm{N}-\mathrm{Ar}\right), 3.29$ (s, $\left.1 \mathrm{H}, \mathrm{CH}_{2}-\mathrm{N}-\mathrm{Ar}\right), 4.76$ (s, 1H, CH-O), $5.5(\mathrm{~s}, 1 \mathrm{H}, \mathrm{OH}), 5.81$ (s, 1H, NH-Ar), 6.68 (s, 1H, Ar-H), 6.83 (s, 1H, Ar-H), 7.11 (s, 2H, Ar-H), 7.19 (s, 2H, Ar-H), 7.26 (s, 1H, Ar-H), 7.35 (s, 2H, Ar-H), 7.42 (s, 2H, Ar-H), 7.54 (s, 1H, Ar-H), $7.59(\mathrm{~s}, 1 \mathrm{H}, \mathrm{Ar}-\mathrm{H}), 9.74(\mathrm{~s}, 1 \mathrm{H}, \mathrm{CO}-\mathrm{NH}) ;{ }^{13} \mathrm{C}-\mathrm{NMR}$ (DMSO-d6): $\delta$ 21.71, 51.77, 71.28, 111.43, 115.36, 115.86, 118.03, 121.37, 124.64, 126.55, 127.49, 128.53, 128.86, 129.29, 138.14, 144.62, 149.21, 166.77; MS (EI) m/z: 348 $(\mathrm{M}+, 100 \%)$.

\section{$\mathrm{N}$-(4-chlorophenyl)-3-(2-hydroxy-}

2-phenylethylamino)benzamide (106)

Prepared by reducing $59(0.5 \mathrm{~g}, 1.4 \mathrm{mmol})$ using sodium borohydride $(0.06 \mathrm{~g}, 1.68 \mathrm{mmol})$ to yield $0.37 \mathrm{~g}(74 \%)$ of 106 as white crystals. Melting point $175^{\circ} \mathrm{C}-177^{\circ} \mathrm{C}$; IR $3,336,3,028,1,651,1,334,1,089 \mathrm{~cm}^{-1} ;{ }^{1} \mathrm{H}$ NMR $(400 \mathrm{mHz}$, DMSO-d6): $\delta 3.18\left(\mathrm{~m}, 1 \mathrm{H}, \mathrm{CH}_{2}-\mathrm{N}-\mathrm{Ar}\right), 4.77(\mathrm{t}, 1 \mathrm{H}, \mathrm{CH}-\mathrm{O}$, $\mathrm{J}=3.7 \mathrm{~Hz}), 5.48(\mathrm{~d}, 1 \mathrm{H}, \mathrm{OH}, \mathrm{J}=4.2 \mathrm{~Hz}), 5.82(\mathrm{t}, 1 \mathrm{H}, \mathrm{NH}-\mathrm{Ar}$, $\mathrm{J}=5.6 \mathrm{~Hz}), 6.84$ (d, 1H, Ar-H, J=8 Hz), 7.10 (m, 2H, Ar-H), 7.21 (t, 1H, Ar-H, J=7.8 Hz), 7.26 (t, 1H, Ar-H, J=7.1 Hz), $7.38(\mathrm{~m}, 6 \mathrm{H}, \mathrm{Ar}-\mathrm{H}), 7.8(\mathrm{~d}, 2 \mathrm{H}, \mathrm{Ar}-\mathrm{H}, \mathrm{J}=8.8 \mathrm{~Hz}), 10.18(\mathrm{~s}, 1 \mathrm{H}$, CO-NH); ${ }^{13} \mathrm{C}-\mathrm{NMR}$ (DMSO-d6): $\delta 51.78,71.32,111.50$, 115.37, 116.01, 122.31, 126.56, 127.50, 128.53, 128.95, $129.35,136.11,138.81,144.61,149.27,166.88$; MS (EI) $\mathrm{m} / \mathrm{z}: 367(\mathrm{M}+, 100 \%)$.

\section{N-(3-chlorophenyl)-3-(2-hydroxy-2- phenylethylamino)benzamide (107)}

Prepared by reducing $61(0.5 \mathrm{~g}, 1.4 \mathrm{mmol})$ using sodium borohydride $(0.062 \mathrm{~g}, 1.68 \mathrm{mmol})$ to yield $0.39 \mathrm{~g}$ (78\%) of 107 as yellow oil. This was purified using preparative thin layer chromatography before characterization. IR 3,305, 3,282, $3,263,2,918,1,643,1,311,1,060 \mathrm{~cm}^{-1}$; ${ }^{1} \mathrm{H}$ NMR $(400 \mathrm{mHz}$, DMSO-d6): $\delta 3.19$ (m, 1H, $\left.\mathrm{CH}_{2}-\mathrm{N}-\mathrm{Ar}\right), 3.29\left(\mathrm{~m}, 1 \mathrm{H}, \mathrm{CH}_{2}-\right.$ N-Ar), 4.77 (s, 1H, CH-O), 5.5 (d, 1H, OH, J=4.5 Hz), 5.86 (d, 1H, NH-Ar, J=4.9 Hz), 6.85 (d, 1H, Ar-H, J=5.7 Hz), 7.13 (m, 3H, Ar-H), 7.21 (t, 1H, Ar-H, J=6.7 Hz), 7.26 (d, 1H, Ar-H, 
$\mathrm{J}=6.8 \mathrm{~Hz}), 7.34(\mathrm{~m}, 3 \mathrm{H}, \mathrm{Ar}-\mathrm{H}), 7.38$ (d, 2H, Ar-H, J=7.9 Hz), 7.69 (d, 3H, Ar-H, J=5.8 Hz), 7.95 (s, 1H, Ar-H), 10.22 (s, 1H, CO-NH); ${ }^{13} \mathrm{C}-\mathrm{NMR}$ (DMSO-d6): $\delta$ 51.75, 71.29, 111.46 , $115.36,116.11,119.07,120.13,123.62,126.55,127.09$, $128.53,129.24,130.75,133.38,135.95,141.32,144.60$, 149.27, 167.05; MS (EI) m/z: 367 (M+, 100\%).

\section{3-(2-hydroxy-2-phenylethylamino)-}

\section{$\mathrm{N}-(4-m e t h o x y p h e n y l)$ benzamide ( 108$)$}

Prepared by reducing $63(0.5 \mathrm{~g}, 1.4 \mathrm{mmol})$ using sodium borohydride $(0.063 \mathrm{~g}, 1.68 \mathrm{mmol})$ to yield $0.35 \mathrm{~g}(70 \%)$ of 108 as white crystals. Melting point $153^{\circ} \mathrm{C}-155^{\circ} \mathrm{C}$; IR 3,406 , 3,334, 3,250, 3,028, 1,649, 1,334, 1,234, 1,035 $\mathrm{cm}^{-1} ;{ }^{1} \mathrm{H} \mathrm{NMR}$ (400 mHz, DMSO-d6): $\delta 3.18\left(\mathrm{~m}, 1 \mathrm{H}, \mathrm{CH}_{2}-\mathrm{N}-\mathrm{Ar}\right), 3.27$ (m, 1H, $\left.\mathrm{CH}_{2}-\mathrm{N}-\mathrm{Ar}\right), 3.74$ (s, 3H CH $\mathrm{H}_{3}$ ), 4.77 (s, 2H, CH-O), 5.50 (s, 1H, OH), 5.79 (s, 1H, NH-Ar), 6.82 (d, 1H, Ar-H, $\mathrm{J}=7.4 \mathrm{~Hz}), 6.91$ (d, 2H, Ar-H, J=8.3 Hz), 7.11 (m, 2H, Ar-H), 7.19 (t, 2H, Ar-H, J=7.7 Hz), 7.26 (t, 1H, Ar-H, J=7 Hz), 7.35 (t, 2H, Ar-H, J=7.1 Hz), 7.42 (d, 2H, Ar-H, J=7.1 Hz), 7.64 (d, 2H, Ar-H, J=8.3 Hz), 9.93 (s, 1H, CO-NH); ${ }^{13} \mathrm{C}-\mathrm{NMR}$ (DMSO-d6): $\delta 51.80,55.68,71.29,111.41,114.18,115.30$, $115.75,122.45,126.56,127.50,128.53,129.26,132.89$, 136.46, 144.63, 149.21, 155.91, 166.32; MS (EI) m/z: 363 $(\mathrm{M}+, 54 \%)$.

\section{3-(2-hydroxy-2-phenylethylamino)-}

\section{$\mathrm{N}$-(3-methoxyphenyl)benzamide ( 109)}

Prepared by reducing 65 (0.5 g, $1.4 \mathrm{mmol})$ using sodium borohydride $(0.063 \mathrm{~g}, 1.68 \mathrm{mmol})$ to yield $0.2 \mathrm{~g}(40 \%)$ of 109 as light brown oil. This was purified using preparative thin layer chromatography before characterization. IR 3,340, 3,288, 3,030, 1,645, 1,332, 1,047 $\mathrm{cm}^{-1} ;{ }^{1} \mathrm{H}$ NMR (400 mHz, DMSO-d6): $\delta 3.33\left(\mathrm{~m}, 1 \mathrm{H}, \mathrm{CH}_{2}-\mathrm{N}-\mathrm{Ar}\right), 3.38\left(\mathrm{~m}, 1 \mathrm{H}, \mathrm{CH}_{2}-\right.$ N-Ar), 4.78 (m, 1H, CH-O), 5.51 (d, 1H, OH, J=4.4 Hz), 5.83 (t, 1H, NH-Ar, J=5.7 Hz), 6.67 (dd, 1H, Ar-H, J=8.2, $3.3 \mathrm{~Hz}$ ), 6.85 (d, 1H, Ar-H, J=7.7 Hz), 7.11 (d, 1H, Ar-H, J=7.8 Hz), 7.13 (s, 1H, Ar-H), 7.24 (m, 3H, Ar-H), 7.35 (m, 4H, Ar-H), 7.43 (d, 2H, Ar-H, J=7.3 Hz), 10.04 (s, 1H, CO-NH); ${ }^{13} \mathrm{C}-$ NMR (DMSO-d6): $\delta$ 51.77, 55.49, 71.30, 106.50, 109.43, $111.48,113.03,115.34,115.89,126.32,127.51,128.54$, $129.31,129.80,136.38,141.03,144.63,149.23,159.90$, 166.82; MS (EI) m/z: $363(\mathrm{M}+, 100 \%)$.

\section{N-[4-(I-hydroxyethyl)phenyl]-3-(2-hydroxy-}

\section{2-phenylethylamino)benzamide (I I0)}

Prepared by reducing 67 (0.5 g, $1.6 \mathrm{mmol})$ using sodium borohydride $(0.144 \mathrm{~g}, 3.84 \mathrm{mmol})$ to yield $0.25 \mathrm{~g}(59 \%)$ of 110 as light brown oil. This was purified using preparative thin layer chromatography before characterization. IR 3,309, 3,296, 3,277, 2,924, 1,654, 1,321 $\mathrm{cm}^{-1} ;{ }^{1} \mathrm{H}$ NMR (400 mHz, DMSO-d6): $\delta 1.3$ (d, 3H, $\left.\mathrm{CH}_{3}, \mathrm{~J}=6.4 \mathrm{~Hz}\right), 3.17$ (m, 1H, $\left.\mathrm{CH}_{2}-\mathrm{N}-\mathrm{Ar}\right), 3.27\left(\mathrm{~m}, 1 \mathrm{H}, \mathrm{CH}_{2}-\mathrm{N}-\mathrm{Ar}\right), 4.68(\mathrm{~m}$, $1 \mathrm{H}, \mathrm{CH}-\mathrm{OH}), 4.76$ (m, 1H, CH-OH), 5.09 (d, 1H, OH, $\mathrm{J}=4.2 \mathrm{~Hz}$ ), 5.51 (d, 1H, OH, J=4.2 Hz), 5.82 (s, 1H, NH-Ar), 6.82 (d, 1H, Ar-H, J=8.1 Hz), 7.09 (s, 1H, Ar-H), 7.11 (d, 1H, Ar-H, J=5.6 Hz), 7.19 (t, 1H, Ar-H, J=7.8 Hz), 7.25 (t, 1H, Ar-H, J=7.3 Hz), 7.28 (d, 2H, Ar-H, J=8.6 Hz), 7.34 (t, 2H, Ar-H, J=7.5 Hz), 7.41 (d, 2H, Ar-H, J=7.2 Hz), 7.67 (d, 2H, Ar-H, J=8.5 Hz), 10.02 (s, 1H, NH-CO); ${ }^{13} \mathrm{C}-$ NMR (DMSO-d6): $\delta$ 17.85, 29.83, 55.18, 71.67, 74.68, $114.85,118.75,119.21,123.97,129.31,129.95,130.90$, $131.93,132.69,139.80,141.62,146.42,148.02,152.61$, 170.00; MS (EI) m/z: 377 (M+, 100\%).

\section{4-[2-hydroxy-2-(3-methoxyphenyl)ethylamino]-} $\mathrm{N}$-phenylbenzamide (I I I)

Prepared by reducing 79 (0.5 g, $1.4 \mathrm{mmol})$ using sodium borohydride $(0.063 \mathrm{~g}, 1.68 \mathrm{mmol})$ to yield $0.37 \mathrm{~g}(74 \%)$ of 111 as white crystals. Melting point $126^{\circ} \mathrm{C}-127^{\circ} \mathrm{C}$; IR $3,417,3,369,3,340,3,049,1,631,1,316,1,055 \mathrm{~cm}^{-1} ;{ }^{1} \mathrm{H}$ NMR (400 mHz, DMSO-d6): $\delta 3.22\left(\mathrm{~m}, 1 \mathrm{H}, \mathrm{CH}_{2}-\mathrm{N}-\mathrm{Ar}\right)$, 3.31 (m, 1H, $\left.\mathrm{CH}_{2}-\mathrm{N}-\mathrm{Ar}\right), 4.75$ (d, 1H, CH-O, J=3.6 Hz), 5.57 (d, 1H, OH, J=4.3 Hz), 6.34 (t, 2H, NH-Ar, J=5.2 Hz), 6.71 (d, 2H, Ar-H, J=8.5 Hz), 6.84 (d, 1H, Ar-H, J=8 Hz), 7.00 (s, 2H, Ar-H), 7.05 (t, 1H, Ar-H, J=7.3 Hz), 7.26 (d, 1H, Ar-H, $\mathrm{J}=8 \mathrm{~Hz}), 7.32(\mathrm{t}, 2 \mathrm{H}, \mathrm{Ar}-\mathrm{H}, \mathrm{J}=7.8 \mathrm{~Hz}), 7.78(\mathrm{t}, 4 \mathrm{H}, \mathrm{Ar}-\mathrm{H}$, $\mathrm{J}=8.3 \mathrm{~Hz}$ ), 9.81 (s, 1H, CO-NH); ${ }^{13} \mathrm{C}-\mathrm{NMR}$ (DMSO-d6): $\delta 51.26,55.46,71.22,111.52,112.13,112.98,118.77,120.66$, $121.60,123.41,128.94,129.61,129.78,140.25,146.15$, 152.09, 159.68, 165.78; MS (EI) m/z: 363 (M+, 100\%).

\section{4-[2-hydroxy-2-(4-methoxyphenyl)ethylamino]-} $\mathrm{N}$-phenylbenzamide (I I 2)

Prepared by reducing $80(0.25 \mathrm{~g}, 0.7 \mathrm{mmol})$ using sodium borohydride $(0.032 \mathrm{~g}, 0.84 \mathrm{mmol})$ to yield $0.16 \mathrm{~g}(65 \%)$ of 112 as white crystals. Melting point $148^{\circ} \mathrm{C}-150^{\circ} \mathrm{C}$; IR 3,404, 3,332, 3,053, 1,602, 1,323, 1,026 cm ${ }^{-1} ;{ }^{1} \mathrm{H}$ NMR $(400 \mathrm{mHz}$, DMSO-d6): $\delta 3.20\left(\mathrm{~m}, 1 \mathrm{H}, \mathrm{CH}_{2}-\mathrm{N}-\mathrm{Ar}\right), 3.35\left(\mathrm{~m}, 1 \mathrm{H}, \mathrm{CH}_{2}-\right.$ N-Ar), 3.74 (s, 3H, $\mathrm{CH}_{3}$ ), 4.7 (d, 1H, CH-O, J=3.1 Hz), 5.42 (d, 1H, OH, J=2.8 Hz), 6.26 (s, 1H, NH-Ar), 6.68 (d, 2H, Ar-H, J=8 Hz), 6.91 (d, 2H, Ar-H, J=7.8 Hz), 7.03 (t, 1H, Ar-H, J=7.2 Hz), 7.31 (m, 4H, Ar-H), 7.76 (m, 4H, Ar-H), 9.77 (s, 1H, CO-NH); ${ }^{13} \mathrm{C}-\mathrm{NMR}$ (DMSO-d6): $\delta 51.38,55.56$, 70.85, 111.45, 113.96, 120.63, 121.53, 123.38, 127.72, 128.94, 129.78, 136.47, 140.29, 152.13, 158.91, 165.76; MS (EI) $\mathrm{m} / \mathrm{z}: 363(\mathrm{M}+, 100 \%)$. 
4-[2-hydroxy-2-(2-methoxyphenyl)ethylamino]$\mathrm{N}$-phenylbenzamide (I I 3)

Prepared by reducing $82(0.5 \mathrm{~g}, 1.4 \mathrm{mmol})$ using sodium borohydride $(0.065 \mathrm{~g}, 1.68 \mathrm{mmol})$, to yield $0.13 \mathrm{~g}(26 \%)$ of 113 as yellow crystals. Melting point $140^{\circ} \mathrm{C}-142^{\circ} \mathrm{C}$; IR 3,400, 3,323, 3,057, 1,643, 1,321, 1,051 cm ${ }^{-1} ;{ }^{1} \mathrm{H}$ NMR (400 mHz, DMSO-d6): $\delta 2.95$ (s, 1H, $\mathrm{CH}_{2}-\mathrm{N}-\mathrm{Ar}$ ), 3.16 (s, $\left.1 \mathrm{H}, \mathrm{CH}_{2}-\mathrm{N}-\mathrm{Ar}\right), 3.88$ (s, 3H, $\left.\mathrm{CH}_{3}-\mathrm{O}-\mathrm{Ar}\right), 5.09$ (s, 1H, CH-O), $5.36(\mathrm{~s}, 1 \mathrm{H}, \mathrm{OH}), 6.50(\mathrm{~s}, 1 \mathrm{H}, \mathrm{NH}-\mathrm{Ar})$, $6.75\left(\mathrm{~d}, 2 \mathrm{H}, \mathrm{CH}_{2}, \mathrm{~J}=6.8 \mathrm{~Hz}\right), 7.02(\mathrm{~m}, 3 \mathrm{H}, \mathrm{Ar}-\mathrm{H}), 7.25$ (d, 1H, Ar-H, J=6.6 Hz), 7.32 (t, 2H, Ar-H, J=7.3 Hz), 7.51 (d, 1H, Ar-H, J=6.2 Hz), 7.77 (d, 2H, Ar-H, J=8.2 Hz), 7.8 (d, 2H, Ar-H, J=8.4 Hz), 9.79 (s, CO-NH); ${ }^{13} \mathrm{C}-\mathrm{NMR}$ (DMSO-d6): $\delta 54.54,58.91,64.33,109.60,110.05,119.26$, $119.49,120.01,122.00,125.45,127.13,127.58,128.41$, 130.93, 138.95, 150.87, 154.75, 164.43; MS (EI) m/z: 363 $(\mathrm{M}+, 100 \%)$.

\section{4-[2-(3-bromophenyl)-2-hydroxyethylamino]- $\mathrm{N}$-phenylbenzamide (I I4)}

Prepared by reducing $83(0.5 \mathrm{~g}, 1.3 \mathrm{mmol})$ using sodium borohydride $(0.057 \mathrm{~g}, 1.56 \mathrm{mmol})$ to yield $0.28 \mathrm{~g}(56 \%)$ of 114 as white crystals. Melting point $157^{\circ} \mathrm{C}-159^{\circ} \mathrm{C}$; IR 3,375, 3,350, 3,061, 1,614, 1,321, 1,053 $\mathrm{cm}^{-1} ;{ }^{1} \mathrm{H}$ NMR (400 mHz, DMSO-d6): $\delta 3.22$ (d, 1H, $\mathrm{CH}_{2}-\mathrm{N}-\mathrm{Ar}, \mathrm{J}=18.2 \mathrm{~Hz}$ ), 3.35 (d, 1H, $\left.\mathrm{CH}_{2}-\mathrm{N}-\mathrm{Ar}, \mathrm{J}=19.8 \mathrm{~Hz}\right), 4.77$ (d, 1H, CH-O, $\mathrm{J}=4.4 \mathrm{~Hz}), 5.72(\mathrm{~s}, 1 \mathrm{H}, \mathrm{OH}), 6.36$ (s, 1H, NH-Ar), 6.71 (d, 2H, Ar-H, J=7.9 Hz), 7.04 (t, 1H, Ar-H, J=6.8 Hz), 7.32 (t, 3H, Ar-H, J=7.6 Hz), 7.42 (d, 1H, Ar-H, J=7.4 Hz), 7.47 (d, 1H, Ar-H, J=7.6 Hz), 7.62 (s, 1H, Ar-H), 7.77 (t, 4H, Ar-H, $\mathrm{J}=7.6 \mathrm{~Hz}$ ), 9.80 (s, 1H, CO-NH); ${ }^{13} \mathrm{C}-\mathrm{NMR}$ (DMSO-d6): $\delta$ 45.75, 65.40, 106.30, 115.40, 116.40, 116.45, 116.79, 118.14, $120.49,123.70,124.10,124.51,125.11,125.50,135.02$, 142.08, 146.79, 160.49; MS (EI) m/z: 412 (M+, 100\%).

\section{4-[2-(4-fluorophenyl)-2-hydroxyethylamino]-} $\mathrm{N}$-phenylbenzamide (I I5)

Prepared by reducing $84(0.5 \mathrm{~g}, 1.4 \mathrm{mmol})$ using sodium borohydride $(0.063 \mathrm{~g}, 1.68 \mathrm{mmol})$ to yield $0.41 \mathrm{~g}(82 \%)$ of 115 as white crystals. Melting point $174^{\circ} \mathrm{C}-176^{\circ} \mathrm{C}$; IR 3,371, 3,350, 3,026, 1,593, 1,323, 1,051 $\mathrm{cm}^{-1} ;{ }^{1} \mathrm{H}$ NMR $(400 \mathrm{mHz}$, DMSO-d6): $\delta 3.20$ (m, 1H, $\left.\mathrm{CH}_{2}-\mathrm{N}-\mathrm{Ar}\right), 3.28\left(\mathrm{~m}, 1 \mathrm{H}, \mathrm{CH}_{2}-\right.$ $\mathrm{N}-\mathrm{Ar}), 4.77$ (s, 1H, CH-O), 5.62 (s, 1H, OH), 6.34 (s, 1H, NH-Ar), 6.69 (d, 2H, Ar-H, J=8.4 Hz), 7.04 (t, 1H, Ar-H, $\mathrm{J}=7.1 \mathrm{~Hz}), 7.17(\mathrm{t}, 2 \mathrm{H}, \mathrm{Ar}-\mathrm{H}, \mathrm{J}=8.7 \mathrm{~Hz}), 7.31$ (t, 2H, Ar-H, $\mathrm{J}=7.6 \mathrm{~Hz}), 7.46(\mathrm{t}, 2 \mathrm{H}$, Ar-H, J=6.1 Hz), 7.77 (t, 4H, Ar-H, $\mathrm{J}=6.9 \mathrm{~Hz}), 9.80$ (s, 1H,CO-NH); ${ }^{13} \mathrm{C}-\mathrm{NMR}$ (DMSO-d6): $\delta$ 49.86, 69.31, 110.14, 113.88 (C-F, J=83.8 Hz), 119.31,
120.22, 122.07, 127.15 (C-F, J=31.9 Hz), 127.60, 128.42, 138.89, 139.07 (C-F, J=138.3 Hz),150.73, 160.49 (C-F, $\mathrm{J}=963 \mathrm{~Hz})$ 164.42; MS (EI) m/z: 351 (M+, 100\%).

\section{4-[2-hydroxy-2-(3-nitrophenyl)ethylamino]-} $\mathrm{N}$-phenylbenzamide (I I6)

Prepared by reducing $85(0.25 \mathrm{~g}, 0.7 \mathrm{mmol})$ using sodium borohydride $(0.032 \mathrm{~g}, 0.84 \mathrm{mmol})$ to yield $0.19 \mathrm{~g}(76 \%)$ of 116 as light yellow crystals. Melting point $128^{\circ} \mathrm{C}-130^{\circ} \mathrm{C}$; IR 3,367, 3,329, 3,053, 1,620, 1,514, 1,321 cm ${ }^{-1}$; ${ }^{1} \mathrm{H}$ NMR (400 mHz, DMSO-d6): $\delta 3.19\left(\mathrm{~m}, 1 \mathrm{H}, \mathrm{CH}_{2}-\mathrm{N}-\mathrm{Ar}\right.$ ), 3.28 (m, 1H, $\left.\mathrm{CH}_{2}-\mathrm{N}-\mathrm{Ar}\right), 4.77$ (s, 2H, CH-O), $5.52(\mathrm{~s}, 1 \mathrm{H}, \mathrm{OH})$, 5.88 (s, 1H, NH-Ar), 6.85 (d, 1H, Ar-H, J=7.8 Hz), 7.1 (d, 2H, Ar-H, J=10.7 Hz), 7.21 (t, 1H, Ar-H, J=7.8 Hz), 7.26 (t, 1H, Ar-H, J=7.2 Hz), 7.35 (t, 2H, Ar-H, J=7.4 Hz), 7.41 (t, 3H, Ar-H, $\mathrm{J}=9.1 \mathrm{~Hz}), 7.81(\mathrm{~d}, 2 \mathrm{H}, \mathrm{Ar}-\mathrm{H}, \mathrm{J}=8.8 \mathrm{~Hz}), 10.22(\mathrm{~s}, 1 \mathrm{H}, \mathrm{CO}-\mathrm{NH})$; ${ }^{13} \mathrm{C}-\mathrm{NMR}$ (DMSO-d6): $\delta$ 45.52, 65.14, 106.30, 115.38, $116.03,116.49,117.25,118.15,123.69,124.50,124.82$, 128.23, 134.99, 141.54, 142.95, 146.73, 160.44; MS (EI) m/z: $378(\mathrm{M}+, 100 \%)$.

\section{4-[2-(4-bromophenyl)-2-hydroxyethylamino]-} $\mathrm{N}$-phenylbenzamide (I I7)

Prepared by reducing $86(0.5 \mathrm{~g}, 1.2 \mathrm{mmol})$ using sodium borohydride $(0.056 \mathrm{~g}, 1.44 \mathrm{mmol})$, to yield $0.35 \mathrm{~g}(70 \%)$ of 117 as white crystals. Melting point $202^{\circ} \mathrm{C}-204^{\circ} \mathrm{C}$; IR 3,375, 3,061, 1,614, 1,323, 1,072 $\mathrm{cm}^{-1} ;{ }^{1} \mathrm{H}$ NMR (400 mHz, DMSO-d6): $\delta 3.23\left(\mathrm{~m}, 1 \mathrm{H}, \mathrm{CH}_{2}-\mathrm{N}-\mathrm{Ar}\right.$ ), 3.30 (m, 1H, $\left.\mathrm{CH}_{2}-\mathrm{N}-\mathrm{Ar}\right), 4.75$ (s, 1H, CH-O), $5.65(\mathrm{~s}, 1 \mathrm{H}, \mathrm{OH})$, 6.34 (s, 1H, NH-Ar), 6.69 (d, 2H, Ar-H, J=7.4 Hz), 7.04 (t, 1H, Ar-H, J=6.8 Hz), 7.31 (t, 2H, Ar-H, J=7 Hz), 7.38 (d, 2H, Ar-H, J=6.9 Hz), 7.55 (d, 2H, Ar-H, J=6.8 Hz), 7.76 (t, 4H, Ar-H, J=6.8 Hz), 9.78 (s, 1H, NH-CO); ${ }^{13} \mathrm{C}-\mathrm{NMR}$ (DMSO-d6): $\delta 51.01,70.64,111.51,120.54,120.65,121.67$, 123.41, 128.87, 128.94, 129.77, 131.40, 140.24, 143.86, 152.02, 165.75; MS (EI) m/z: 411 (M+, 100\%).

\section{4-[2-(4-chlorophenyl)-2-hydroxyethylamino]- $\mathrm{N}$-phenylbenzamide (I I8)}

Prepared by reducing $88(0.5 \mathrm{~g}, 1.4 \mathrm{mmol})$ using sodium borohydride $(0.062 \mathrm{~g}, 1.68 \mathrm{mmol})$ to yield $0.41 \mathrm{~g}(82 \%)$ of 118 as white crystals. Melting point $198^{\circ} \mathrm{C}-200^{\circ} \mathrm{C}$; IR 3,394, 3,358, 3,321, 3,057, 1,622, 1,321, 1,085 cm-1; ${ }^{1} \mathrm{H}$ NMR (400 mHz, DMSO-d6): $\delta 3.24$ (m, 1H, $\mathrm{CH}_{2}-\mathrm{N}-\mathrm{Ar}$ ), $3.28\left(\mathrm{~m}, 1 \mathrm{H}, \mathrm{CH}_{2}-\mathrm{N}-\mathrm{Ar}\right), 4.78(\mathrm{~s}, 2 \mathrm{H}, \mathrm{Ar}-\mathrm{H}), 5.66(\mathrm{~s}, 1 \mathrm{H}$, $\mathrm{OH}), 6.34$ (s, 1H, NH-Ar), 6.7 (d, 2H, Ar-H, J=7.6 Hz), 7.04 (t, 1H, Ar-H, J=7.3 Hz), 7.31 (t, 2H, Ar-H, J=7.3 Hz), 7.43 (d, 4H, Ar-H, J=7.4 Hz), 7.78 (t, 4H, Ar-H, J=7 Hz), 9.81 
(s, 1H, CO-NH); ${ }^{13} \mathrm{C}-\mathrm{NMR}$ (DMSO-d6): $\delta$ 49.70, 69.28, $110.17,119.33,120.31,122.10,127.14,127.61,128.43$, $130.69,138.86,142.04,150.68,164.44 ; \mathrm{MS}(\mathrm{EI}) \mathrm{m} / \mathrm{z}: 367$ $(\mathrm{M}+, 100 \%)$.

\section{4-(2-hydroxy-2-p-tolylethylamino)-}

N-phenylbenzamide ( I 19)

Prepared by reducing 89 (0.5 g, $1.5 \mathrm{mmol})$ using sodium borohydride $(0.07 \mathrm{~g}, 1.8 \mathrm{mmol})$ to yield $0.33 \mathrm{~g}(66 \%)$ of 119 as white crystals. Melting point $173^{\circ} \mathrm{C}-175^{\circ} \mathrm{C}$; IR 3,387, 3,373, 3,059, 1,622, 1,319 $\mathrm{cm}^{-1}$; ${ }^{1} \mathrm{H} \mathrm{NMR}(400 \mathrm{mHz}$, DMSO-d6): $\delta 2.28$ (s, 3H, $\mathrm{CH}_{3}$ ), 3.18 (s, 1H, $\mathrm{CH}_{2}-\mathrm{N}-\mathrm{Ar}$ ), 3.24 (s, 1H, $\left.\mathrm{CH}_{2}-\mathrm{N}-\mathrm{Ar}\right), 4.71$ (s, 1H,CH-O), 5.47 (s, 1H, $\mathrm{OH}), 6.30$ (s, 1H, NH), 6.68 (d, 2H, Ar-H, J=5.2 Hz), 7.02 (s, 1H, Ar-H), 7.15 (s, 2H, Ar-H), 7.29 (s, 4H, Ar-H), 7.75 (s, 4H, Ar-H), 9.78 (s, 1H, CO-NH); ${ }^{13} \mathrm{C}-\mathrm{NMR}$ (DMSO-d6): $\delta 21.23,71.07,111.46,120.63,121.52,123.40,126.48$, $129.11,129.78,136.57,140.26,141.45,152.1,165.77$; MS (EI) $\mathrm{m} / \mathrm{z}: 348(\mathrm{M}+, 100 \%)$.

\section{4-(2-hydroxy-2-naphthalen-2-ylethylamino)-} $\mathrm{N}$-phenylbenzamide (120)

Prepared by reducing 90 (0.5 g, $1.3 \mathrm{mmol})$ using sodium borohydride $(0.06 \mathrm{~g}, 1.56 \mathrm{mmol})$ to yield $0.39 \mathrm{~g}(78 \%)$ of 120 as off-white crystals. Melting point $187^{\circ} \mathrm{C}-190^{\circ} \mathrm{C}$; IR 3,373, 3,055, 1,620, 1,321 cm ${ }^{-1}$; ${ }^{1} \mathrm{H}$ NMR (400 mHz, DMSO-d6): $\delta 3.33$ (m, 1H, $\left.\mathrm{CH}_{2}-\mathrm{N}-\mathrm{Ar}\right), 3.40\left(\mathrm{~m}, 1 \mathrm{H}, \mathrm{CH}_{2}-\right.$ N-Ar), 4.85 (s, 1H, CH-O), 5.68 (d, 1H, NH, J=2.8 Hz), $6.74(\mathrm{~d}, 2 \mathrm{H}, \mathrm{Ar}-\mathrm{H}, \mathrm{J}=9.8 \mathrm{~Hz}), 7.06$ (t, 1H, Ar-H, J=9.2 Hz), 7.32 (t, 2H, Ar-H, J=7.8 Hz), 7.51 (m, 2H, Ar-H), 7.61 (d, 2H, Ar-H, J=7.8 Hz), 7.77 (d, 2H, Ar-H, J=8.2 Hz), 7.8 (d, 2H, Ar-H, J=8.8 Hz), 7.92 (m, 4H, Ar-H), 9.78 (s, 1H, CO-NH); ${ }^{13}$ C-NMR (DMSO-d6): $\delta$ 50.64, 70.87, 110.98, 120.09, $121.10,122.83,124.48,124.62,125.58,125.98,127.46$, $127.54,127.73,128.39,129.24,132.38,132.79,139.73$, 141.47, 151.58, 165.20; MS (EI) m/z: 383 (M+, 100\%).

\section{DPP-IV enzymatic assay}

The DPP-IV drug discovery kit marketed by Enzo Life Sciences (Farmingdale, New York, NY, USA) was used for this test. This kit includes human recombinant DPP-IV enzyme, chromogenic substrate (H-Gly-Pro-pNA), a calibration standard (p-nitroaniline), an inhibitor (P32/98), and an assay buffer (Tris, $\mathrm{pH} 7.5$ ). The kit was stored at $-70^{\circ} \mathrm{C}$. On the day before testing, the kit components were defrosted in the refrigerator and thawed at room temperature on the day of the test.

The inhibitor and compounds to be tested were all prepared in DMSO solution and then diluted in assay buffer to yield solutions at a concentration of $100 \mu \mathrm{M}$. Next, $5 \mu \mathrm{L}$ of the substrate was diluted in $245 \mu \mathrm{L}$ of assay buffer, and $5 \mu \mathrm{L}$ of the enzyme was diluted in $245 \mu \mathrm{L}$ of assay buffer.

The assay buffer was then distributed in all wells of the plate in the amounts described in Table 1. The plate was then placed in an oven at $37^{\circ} \mathrm{C}$ for 5 minutes to equilibrate. Next, $15 \mu \mathrm{L}$ of the enzyme was added to each well except the blank. The inhibitor and the test compound were then added. The plate was again incubated at $37^{\circ} \mathrm{C}$ for 10 minutes to allow interaction between the inhibitor and the enzyme. The final step was adding the substrate to start the assay. After that, the plate was read using a microplate reader every $2-3$ minutes for a total of 20 minutes.

\section{Results and discussion Design strategy}

The design of the target compounds in this project involved a combination of computer-aided and traditional drug design. The design of the core structures started with a guide compound (compound 1, see Figure 2) that had a satisfactory inhibitory activity of $28 \%$ against the DDP-4 enzyme. Careful modifications were then introduced based on a thorough inspection of the three-dimensional structure of DPP-IV. The proposed structures were docked inside the active site using specialized in silico software (Discovery Studio 3.5) and the possible binding energies were evaluated using computational calculations that scored and ranked these energies.

The work started with docking of compound 1 inside the active site using CDOCKER docking software (Figure 3), that gave us an idea about the most likely orientation of this compound in the active site of DPP-IV. Based on this pose, it can be easily recognized that the aminobenzamide part of the compound is important for binding since it forms a hydrogen bond with the active site in addition to one $\pi-\pi$ interaction. Based on the proper orientation the compound 1 had adopted by matching the active site geometry and which also fostered by performing a network of interactions with the receptor, the aminobenzamide moiety was adopted as the core building scaffold in this research, with addition of suitable extensions

Table I Amounts of kit components used (in $\mu \mathrm{L}$ )

\begin{tabular}{lllll}
\hline Sample & $\begin{array}{l}\text { Assay } \\
\text { buffer }\end{array}$ & DPP-IV & Inhibitor & Substrate \\
\hline Blank & 50 & 0 & 0 & 50 \\
Negative control & 35 & 15 & 0 & 50 \\
Positive control & 25 & 15 & 10 & 50 \\
Tested compounds & 25 & 15 & 10 & 50 \\
\hline
\end{tabular}

Abbreviation: DPP-IV, dipeptidyl peptidase-IV. 


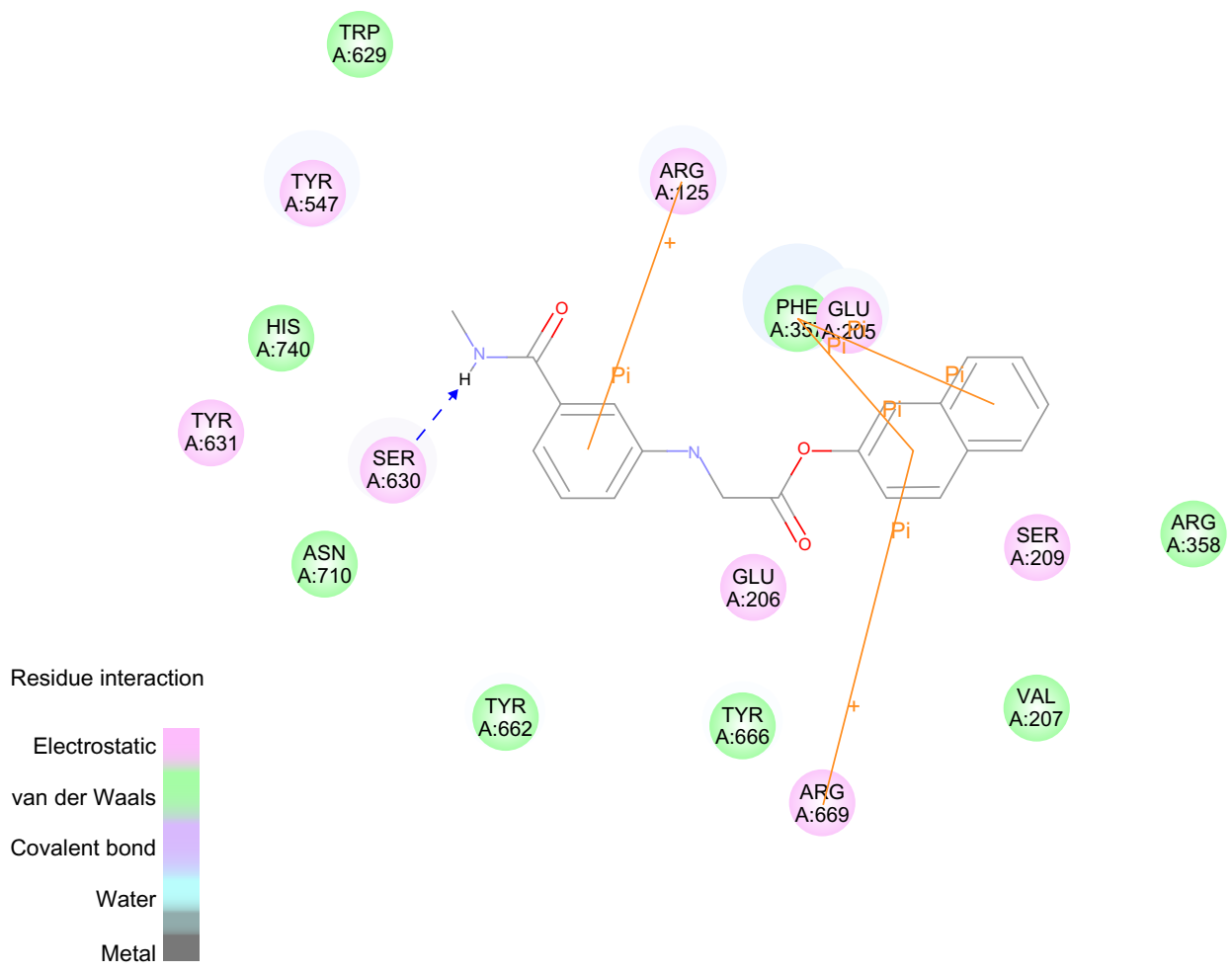

Figure 3 Structure of compound I docked in the active site of the dipeptidyl peptidase-IV enzyme.

to optimize the binding process. The CDOCKER interaction energy (CIE), which represents a numerical estimation of the ligand-receptor interaction energy of compound 1, was 40, while the CDOCKER energy (CE) that, in addition to ligand-receptor interaction, takes into consideration the strain of the ligand when placed inside the active site of the same compound was 21 .

The $\mathrm{S} 1$ pocket (Figure 1A) is known to be important in DPP-IV binding, so enhancing the binding of the compound in this pocket could be very beneficial to its activity. In Figure 3, the methyl group of compound 1 is placed in the $\mathrm{S} 1$ pocket, but this pocket is capable of accommodating larger substitutions. While a phenyl group can form a hydrophobic interaction with this hydrophobic pocket, it can also form a $\pi-\pi$ interaction with some of the amino acids in the same pocket, including tyrosine, tryptophan, and histidine. This was validated when compound 2 was docked and a phenyl formed a bond with His 740 (Figure 4). The CIE of compound 2 was 44 and the CE was 23 , so it can be seen that adding the phenyl group enhanced both values, indicating preferential binding. However, the increase in CIE was more than that in CE, suggesting that addition of the phenyl enhanced binding but at the same time introduced some strain into the compound.

The next strategy adopted for upgrading the activity of compound 1 was to focus on the presence of ester functionality. Esters suffer stability problems during synthesis, storage, and testing, so we opted for bioisosteric replacement of esters with more stable groups. Ketones were the most favorable group due to their ability to function as hydrogen bond acceptors and their planarity (like esters); in addition, they can be easily converted to alcohols, providing an opportunity to study the type of binding which this group can embrace. Ketones are hydrogen bond acceptors and alcohols are hydrogen bond donors, so using these may increase the possibilities for binding, especially with the key amino acids inside the active sites, ie, Glu205 and Glu206.

Bioisosteric replacement of compound 2 with compound 4 (Figure 5) increased the CIE to 45, but was detrimental to the CE, which went down to 19. This means that while the ketone improved binding, it also considerably increased the strain on the ligand inside the active site. Fortunately, bioisosteric replacement of naphthyl with phenyl (compound 4 to compound 5, Figure 5) has spared suitable space to relax compound 5 inside the active site, with a significant improvement in the $\mathrm{CE}$ (from 19 in compound 4 to 28 in compound 5 ). This finding was confirmed when the same bioisosteric replacement was introduced in compound 2 to form compound 3, and in this case the CE increased from 23 to 31 . In both cases, the CIE was about 40 . 


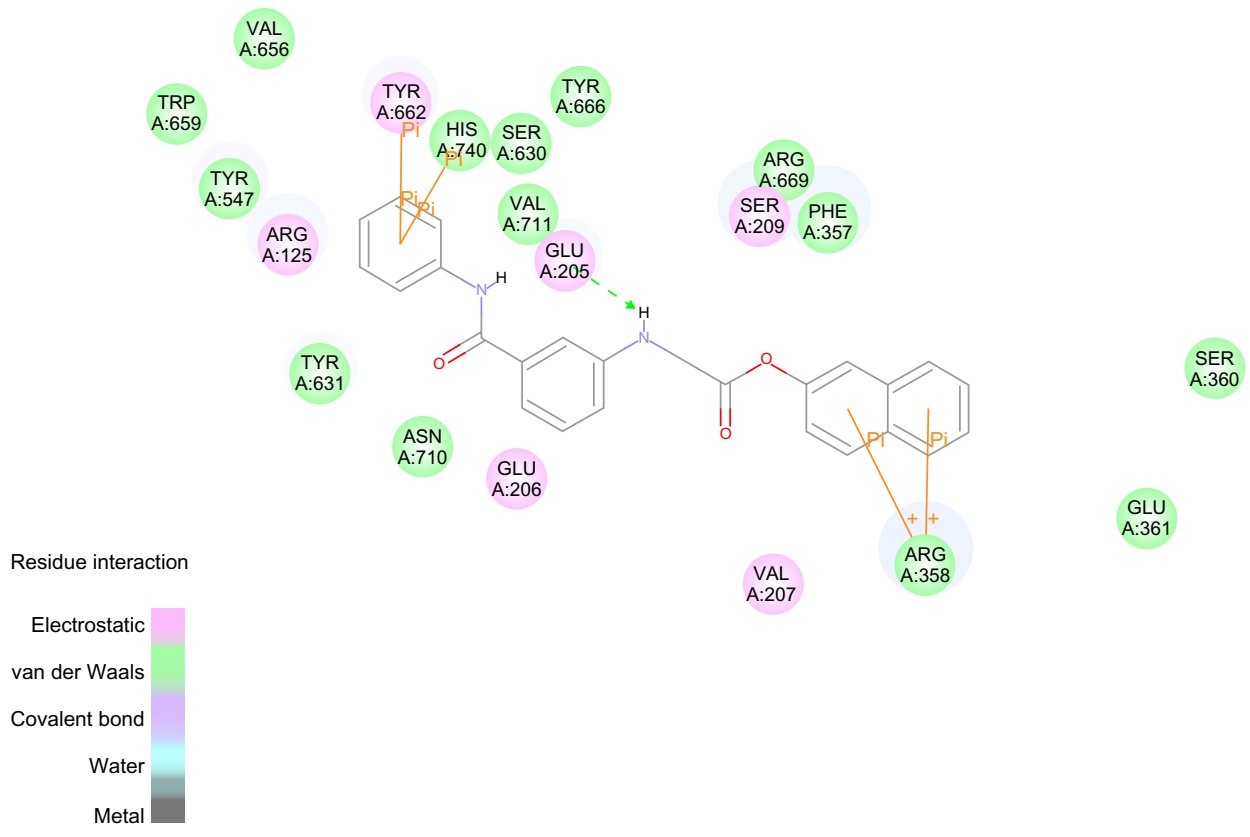

Figure 4 Structure of compound 2 docked inside the active site of the dipeptidyl peptidase-IV enzyme.

The next modification was made to test the effect of changing the aminobenzamide moiety from meta (compound 5) to para (compound 6). There were no changes in the CIE (40) or CE (28) values, suggesting that there is a certain degree of flexibility in the binding.

After confirming the core scaffold by computer-aided programs, substitutions on both phenyls were achieved using a conventional paradigm which is consolidated on choosing hydrophilic and hydrophobic groups combined with selecting groups known to be electron-donating and electronwithdrawing in addition to being connected to phenyl rings at various points. This strategic methodology was used in order to attenuate our reliance solely on one type of software to design a series of inhibitors, which might not be suitable for such types of active sites. A range of substituents was suggested, including: methyl, trifluoromethyl, fluoro, chloro, and bromo substitutions to test the effect of hydrophobicity and size on the binding; and methoxy, carboxylic acid, nitro, and acetyl groups to test the possibility of formation of hydrogen bonds and their placement.

\section{Docking}

As explained earlier, designing the proposed compounds was guided partially by the results of docking. The CDOCKER software in Discovery Studio 3.5 was utilized for docking of these compounds..$^{15}$<smiles>CNC(=O)c1cccc(NCC(=O)Oc2ccc3ccccc3c2)c1</smiles>

Compound 1<smiles>O=C(CNc1cccc(C(=O)Nc2ccccc2)c1)c1ccc2ccccc2c1</smiles>

Compound 4<smiles>O=C(CNc1cccc(C(=O)Nc2ccccc2)c1)Oc1ccc2ccccc2c1</smiles>

Compound 2<smiles>O=C(CNc1cccc(C(=O)Nc2ccccc2)c1)c1ccccc1</smiles>

Compound 5<smiles>O=C(CNc1cccc(C(=O)Nc2ccccc2)c1)Oc1ccccc1</smiles>

Compound 3<smiles>O=C(CNc1ccc(C(=O)Nc2ccccc2)cc1)c1ccccc1</smiles>

Compound 6

Figure 5 Chemical structures of compounds I-6. 
The crystal structures of the DPP-IV enzyme are deposited in the Protein Data Bank. ${ }^{14}$ An initial search of the Protein Data Bank resulted in 66 crystal structures with a variety of substrates. Structure 3D4L was selected for two main reasons. Firstly, it had the best resolution ( $2 \AA)$ of all the structures at the time of downloading, and secondly, the substrate cocrystallized in its active site was similar to that in our proposed scaffold, which contains three phenyl rings linked in a semilinear order.

The crystal structure of the enzyme was then prepared using the "Prepare Protein" protocol in Discovery Studio 3.5. The aim of this protocol was to clean up common problems in the crystal structure of the protein and adapt the protein to be manipulated in the other protocols in Discovery Studio 3.5.

The active site determination step was executed carefully due to the fact that it has a wide opening and this would increase the potential orientations that the ligands can adopt; consequently, this would reduce our reliance on the resulting scores because they are subject to a chain of parameters and there is a possibility that the score would not completely reflect the activity. The availability of 66 crystal structures gives us more insight into the type of binding these ligands could perform, but on the other side, this expands the size of the active site and the docking program would require more time to express the results and be interweaved with less accuracy. All the 66 crystal structures were studied, and the amino acids that formed hydrogen bonds or had $\pi-\pi$ interactions with the substrates were identified. Using these amino acids, the "Define Site From Selection" tool was used to determine the boundaries of the active site. The radius of the active site was then accustomed to $13 \AA$ and manually harmonized to fit the amino acids optimally.

The proposed compounds were drawn using ChemDraw ${ }^{\circledR}$ version $12.0^{16}$ and then prepared using the "Prepare Ligand" protocol. Further, they were docked in the previously defined active site using CDOCKER. CDOCKER basically operates by generating random ligand conformations, and the poses of these conformations are placed in the active site followed by comprehensive rotation to find the best fit. Finally, the candidate poses are refined through minimization and scored. The top ten poses are reported for each compound.

\section{Chemical syntheses}

\section{General scheme for synthesis of target compounds}

The target compounds were basically synthesized according to Figure 6. Where different anilines were coupled to para or meta nitrobenzoyl chlorides, the nitro group in the product was reduced to an amine. The resulting amine was again coupled to several alpha haloacetophenone derivatives. The ketone group in the coupling product was reduced to the corresponding alcohol. During this research, 120 compounds were synthesized, of which 69 were novel compounds. The previously known compounds were characterized using proton NMR and by comparing their melting point with that reported in the literature, while the novel compounds were characterized using proton and carbon NMR, mass spectrometry, and infrared spectroscopy, and their melting points were determined.

\section{Synthesis of substituted 4-nitro-N-phenylbenzamide and 3-nitro-N-phenylbenzamide}

Substituted anilines were allowed to react with 4-nitrobenzoyl chloride (Figure 7) or 3-nitrobenzoyl chloride (Figure 8) in the presence of pyridine at room temperature for 8 hours. ${ }^{17}$ The reaction was followed by thin layer chromatography, and found to be a straightforward reaction with one product.

Dichloromethane was used first as a solvent for this reaction followed by an acid/base washing step, but the yields were lower than expected $(60 \%-75 \%)$. A change of the solvent from dichloromethane to tetrahydrofuran and avoiding the washing step by pouring the reaction into water and then filtering the precipitated product resulted in a considerable increase in yield (88\%-97\%).

The products of this step had all been synthesized previously and reported. Their melting points were compared with those reported in the literature. The ${ }^{1} \mathrm{H}$ NMR spectra of the products were as expected, with the aromatic hydrogens appearing in the 7-8.5 range and a singlet around 10.5 representing the amide hydrogen.

\section{Synthesis of substituted 4-amino- $\mathrm{N}$-phenylbenzamide and 3-amino-N-phenylbenzamide}

Substituted 4-nitro-N-phenylbenzamide (Figure 9) and 3-nitro-N-phenylbenzamide (Figure 10) were reduced using one equivalent of ferrous sulfate and five equivalents of iron. ${ }^{18,19}$ Iron was used in excess because stirring resulted in an uneven distribution of the iron and a tendency to accumulate around the magnetic bar.

The reaction was carried out under reflux overnight. The solvent system was an ethanol/water mixture in a 3:1 ratio. The reaction as reported in the literature uses only water as a solvent, but since both the starting material and the product are insoluble in water, adding ethanol facilitated both the reaction and its workup.

The choice of this reduction method was due to its selectivity for reduction of the nitro group in addition to producing the desired product in high yields. Other methods of reduction include catalytic hydrogenation and metal/acid reductions. 


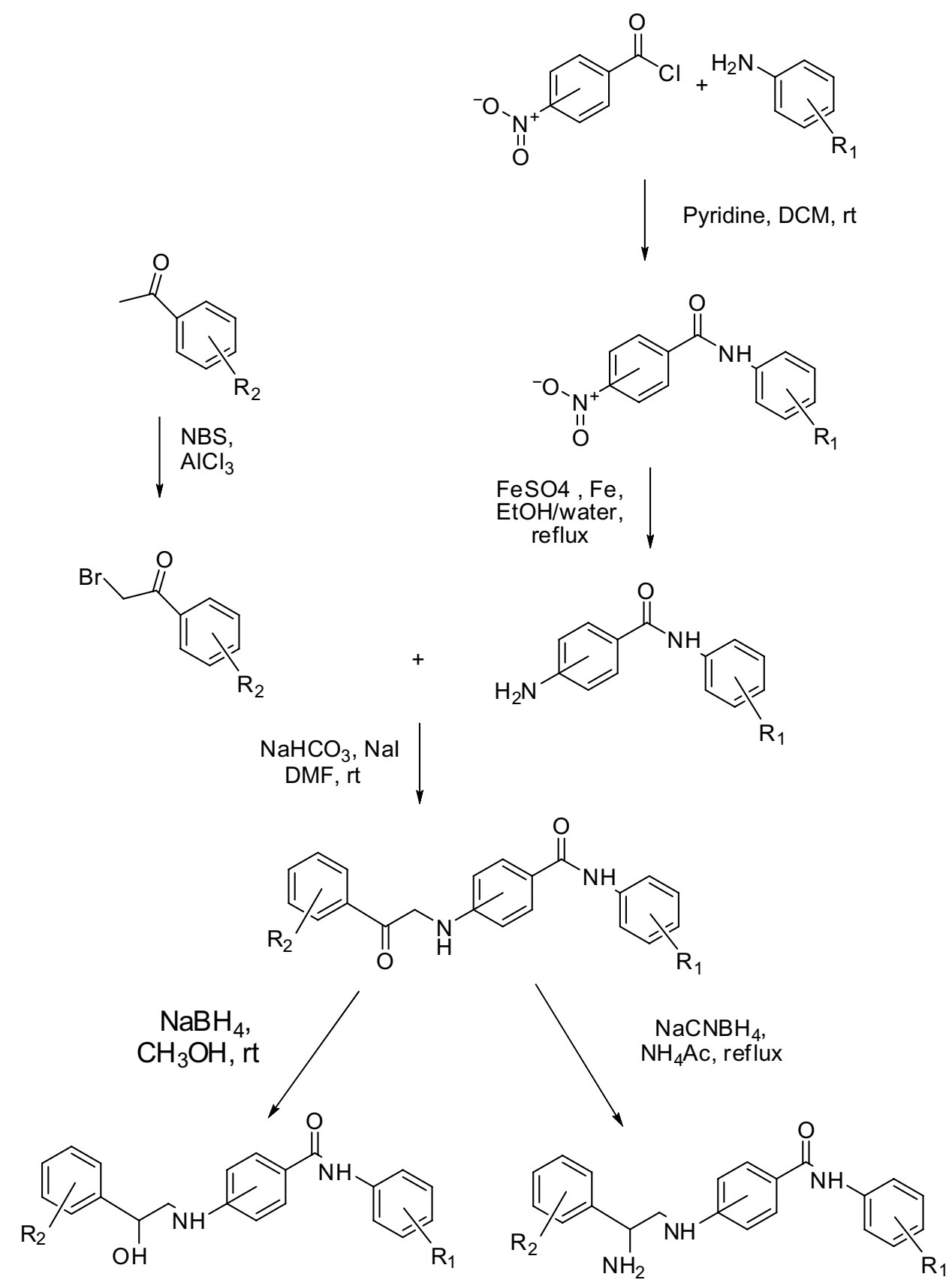

Figure 6 General scheme for the synthesis of target compounds.

However, many of these methods lack chemoselectivity and form several byproducts. ${ }^{20}$

The workup was started by filtration of the reaction while hot to prevent precipitation of the product as the reaction cools down. The filtrate was then evaporated in vacuo and the dry residue was recrystallized from ethanol/water to obtain the pure product in high yields $(88 \%-100 \%)$.

The ${ }^{1} \mathrm{H}$ NMR of the 3-amino-N-phenylbenzamide and 4-amino-N-phenylbenzamide derivatives was similar to that of the 3-nitro-N-phenylbenzamide and 4-nitro-N-phenylbenzamide derivatives, with an additional peak around 5.5, representing the two protons of the newly formed amine. One other difference is the upfield shift of the amide proton from 10.5 in the nitro-N-phenylbenzamides to $9.5-10$ in the amino-N-phenylbenzamides; this shift is brought about by the shielding effect of the electron-donating amino group as compared with the electron-withdrawing nitro group.

\section{Synthesis-substituted 3- and 4-(2-oxo-} 2-phenyl-ethylamino)- $\mathrm{N}$-phenyl-benzamide

The reaction between amino-N-phenylbenzamides and $\alpha$-haloketones was accomplished in the presence of 


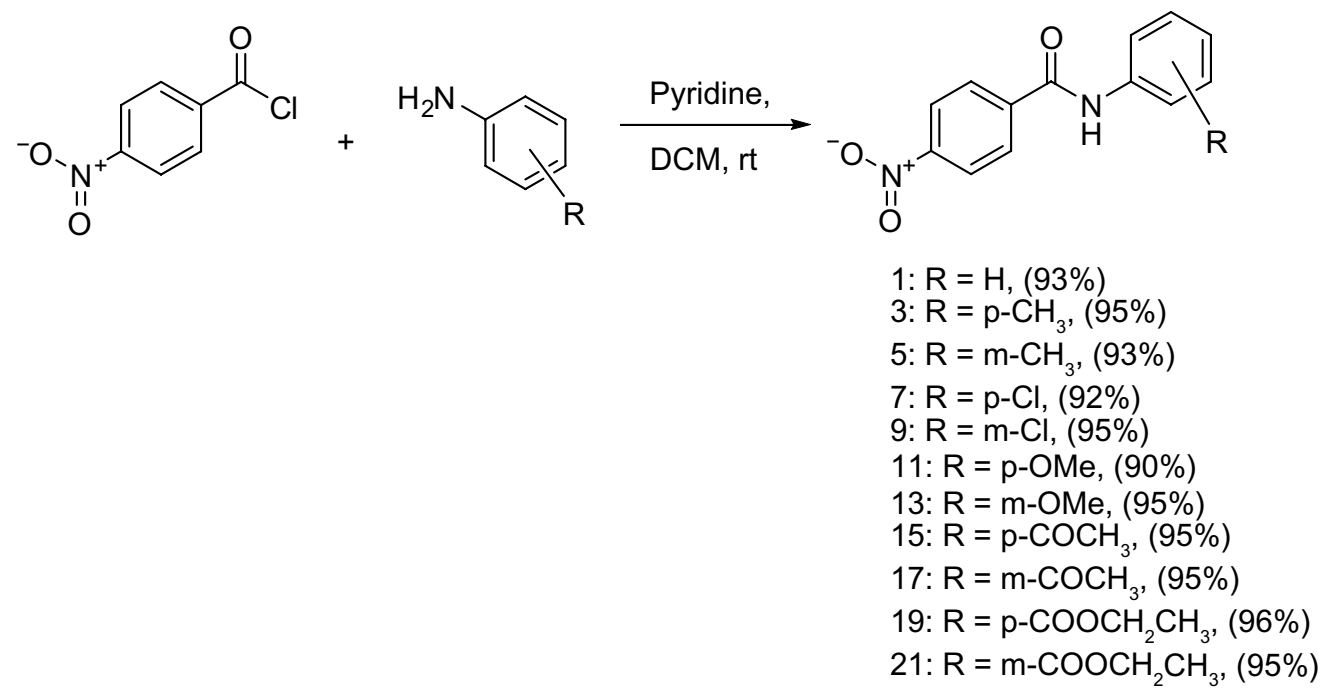

Figure 7 Synthesis of substituted 4-nitro-N-phenylbenzamide.<smiles>O=C(Cl)c1cccc([N+](=O)[O-])c1</smiles><smiles>[R]c1ccc(N)cc1</smiles><smiles>[R]OC1CCCCC1</smiles><smiles>[R]c1cccc(NC(=O)c2cccc([N+](=O)[O-])c2)c1</smiles>

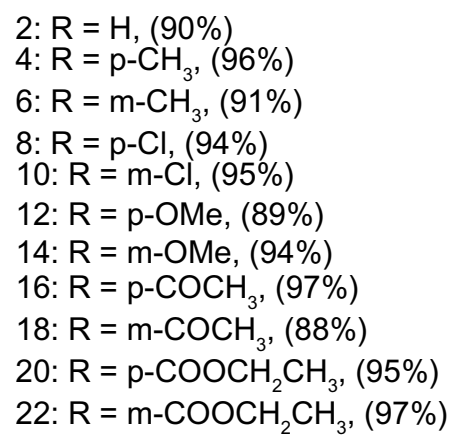

Figure 8 Synthesis of substituted 3-nitro-N-phenylbenzamide.<smiles>[R]c1ccccc1NC(=O)c1ccc([N+](=O)[O-])cc1</smiles>

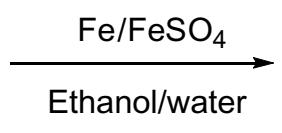<smiles>[R]c1ccccc1NC(=O)c1ccc(N)cc1</smiles>
23: $\mathrm{R}=\mathrm{H},(92 \%)$
25: $\mathrm{R}=\mathrm{p}-\mathrm{CH}_{3},(98 \%)$
$27: \mathrm{R}=\mathrm{m}-\mathrm{CH}_{3},(92 \%)$
29: $\mathrm{R}=\mathrm{p}-\mathrm{Cl},(96 \%)$
31: $\mathrm{R}=\mathrm{m}-\mathrm{Cl},(100 \%)$
33: $R=p-O M e,(96 \%)$
35: $\mathrm{R}=\mathrm{m}-\mathrm{OMe},(92 \%)$
37: $\mathrm{R}=\mathrm{p}-\mathrm{COCH}_{3},(98 \%)$
39: $\mathrm{R}=\mathrm{m}-\mathrm{COCH}_{3},(96 \%)$
41: $\mathrm{R}=\mathrm{p}-\mathrm{COOCH}_{2} \mathrm{CH}_{3},(97 \%)$
43: $\mathrm{R}=\mathrm{m}-\mathrm{COOCH}_{2} \mathrm{CH}_{3},(99 \%)$

Figure 9 Synthesis of substituted 4-amino-N-phenylbenzamide. 
<smiles>[R]c1cccc(NC(=O)c2cccc([N+](=O)[O-])c2)c1</smiles>

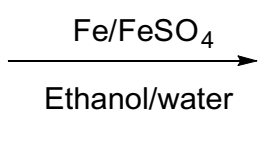<smiles>[R]c1cccc(NC(=O)c2cccc(N)c2)c1</smiles>

Figure 10 Synthesis of substituted 3-amino-N-phenylbenzamide.

potassium bicarbonate, and the reaction was carried out in dimethylformamide at room temperature. The workup consisted of adding water to the reaction, resulting in precipitation of the product, that after being collected and dried was recrystallized from ethanol.

The reaction was straightforward, with the major product being monoalkylation of the amino group. However, byproducts were frequently observed, and their formation was associated with a longer reaction time, a higher temperature, and the use of potassium carbonate instead of potassium bicarbonate, suggesting that these byproducts could be a result of degradation.

Several solvents were tested for this reaction, including tetrahydrofuran, dimethylformamide, acetone, acetonitrile, hexane, and ethanol. A polar aprotic solvent was expected to work best because of the proposed mechanism. Dimethylformamide was our solvent of choice because it increased the rate of the reaction considerably and with fewer byproducts observed on thin layer chromatography.

Increasing the amount of potassium bicarbonate used to two equivalents instead of one and replacing it with potassium carbonate was detrimental to the yield and purity of the product. With $\alpha$-bromoketones, use of potassium bicarbonate was enough to effect the reaction, but sodium iodide was also needed with the $\alpha$-chloroketones and $\alpha$-chloroesters because iodide replaces the chloride ion, forming the more reactive $\alpha$-iodoketones. ${ }^{21}$

The products of this step were either anilinoketones (Figures 11-15) or anilinoesters (Figure 16) depending on the starting $\alpha$-haloketones/esters. The synthesized anilinoketones<smiles>[R]c1cccc(NC(=O)c2ccc(NCC(=O)c3ccccc3)cc2)c1</smiles>

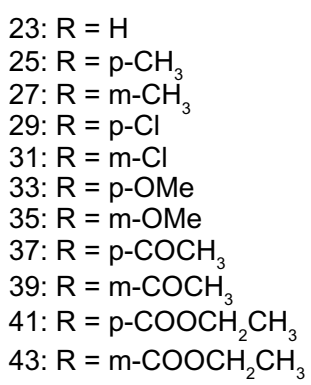

$\mathrm{H} 1$

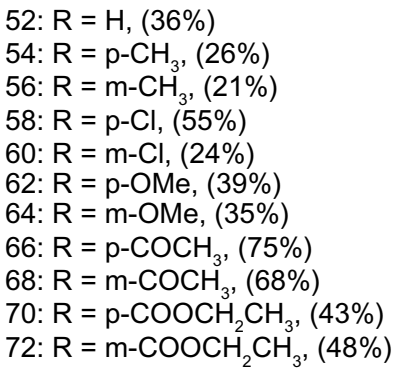

Figure I I Synthesis of anilinoketones from substituted 4-amino-N-phenylbenzamides. 


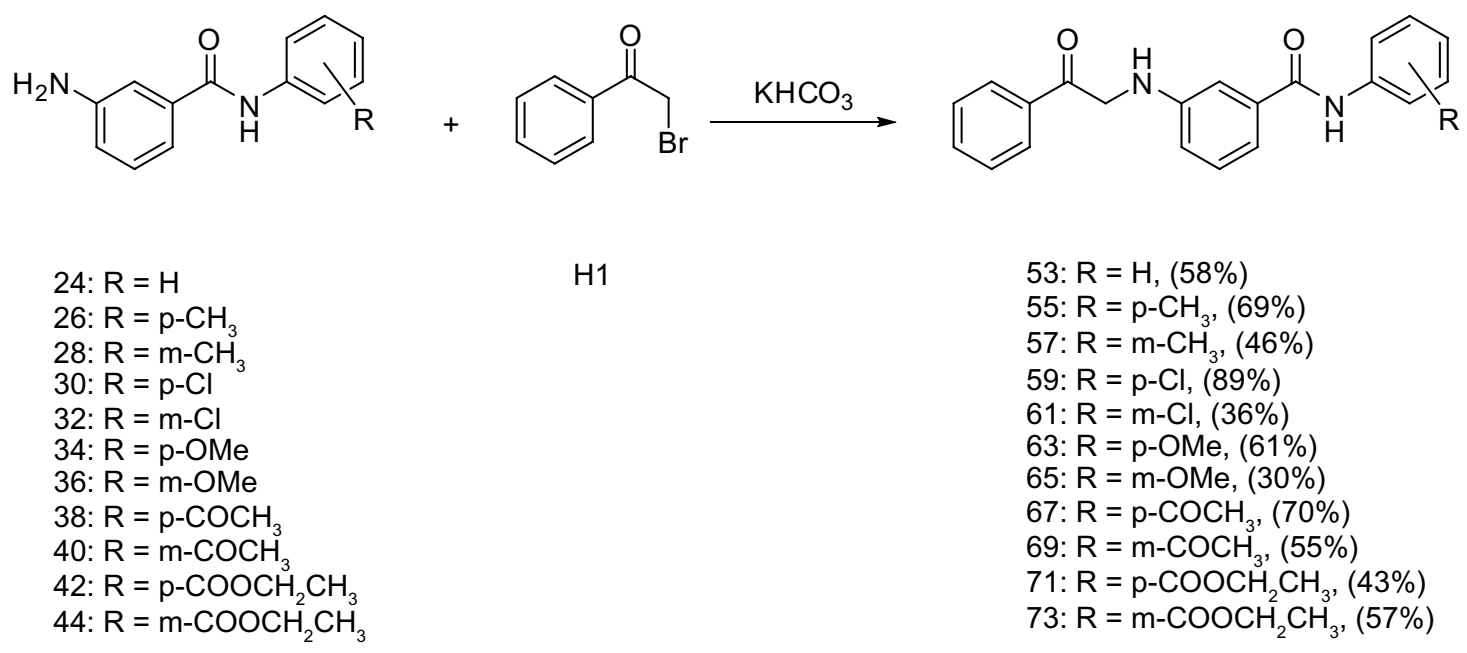

Figure 12 Synthesis of anilinoketones from substituted 3-amino-N-phenylbenzamides.<smiles>[R]c1ccc(C(=O)CBr)cc1</smiles><smiles>Nc1ccc(C(=O)Nc2ccccc2)cc1</smiles><smiles>[R]c1cccc(C(=O)CNc2ccc(C(=O)Nc3ccccc3)cc2)c1</smiles>

45: $\mathrm{R}=\mathrm{p}-\mathrm{NO}$

46: $\mathrm{R}=\mathrm{m}-\mathrm{OCH}_{3}$

47: $\mathrm{R}=\mathrm{p}-\mathrm{OCH}_{3}{ }^{3}$

$\mathrm{H} 2: \mathrm{R}=\mathrm{p}-\mathrm{CN}$

$\mathrm{H} 3: \mathrm{R}=\mathrm{O}-\mathrm{OCH}_{3}$

$\mathrm{H} 4: \mathrm{R}=\mathrm{p}-\mathrm{CF}_{3}$

$\mathrm{H} 5: \mathrm{R}=\mathrm{p}-\mathrm{F}$

$\mathrm{H} 6: \mathrm{R}=\mathrm{m}-\mathrm{NO}_{2}$

$\mathrm{H} 7: \mathrm{R}=\mathrm{p}-\mathrm{Br}$

$\mathrm{H} 8: \mathrm{R}=\mathrm{m}-\mathrm{Br}$

H9: $\mathrm{R}=\mathrm{m}-\mathrm{Cl}$
23
74: $\mathrm{R}=\mathrm{p}-\mathrm{NO}_{2},(58 \%)$

75: $\mathrm{R}=\mathrm{m}-\mathrm{OCH}_{3},(47 \%)$

76: $\mathrm{R}=\mathrm{p}-\mathrm{OCH}_{3},(53 \%)$

$77: \mathrm{R}=\mathrm{p}-\mathrm{CN},(30 \%)$

78: $\mathrm{R}=\mathrm{O}-\mathrm{OCH}_{3},(56 \%)$

79: $\mathrm{R}=\mathrm{p}-\mathrm{CF}_{3},(40 \%)$

80: $\mathrm{R}=\mathrm{p}-\mathrm{F},(59 \%)$

81: $\mathrm{R}=\mathrm{m}-\mathrm{NO}_{2},(27 \%)$

82: $\mathrm{R}=\mathrm{p}-\mathrm{Br},(32 \%)$

83: $\mathrm{R}=\mathrm{m}-\mathrm{Br},(58 \%)$

84: $\mathrm{R}=\mathrm{m}-\mathrm{Cl},(78 \%)$

Figure 13 Synthesis of anilinoketones from several substituted $\alpha$-bromoketones.<smiles>Cc1ccc(C(=O)CCl)cc1</smiles>

48<smiles>Nc1ccc(C(=O)Nc2ccccc2)cc1</smiles>

23<smiles>Cc1ccc(C(=O)CNc2ccc(C(=O)Nc3ccccc3)cc2)cc1</smiles>

$85,(50 \%)$<smiles>O=C(CCl)c1ccc2ccccc2c1</smiles>

49<smiles>Nc1ccc(C(=O)Nc2ccccc2)cc1</smiles>

23<smiles>O=C(CNc1ccc(C(=O)Nc2ccccc2)cc1)c1ccc2ccccc2c1</smiles>

$86,(51 \%)$

Figure 14 Synthesis of anilinoketones from $\alpha$-chloroketones. 
<smiles>COc1ccc(NC(=O)c2ccc(NC(=O)c3ccc(NC(=O)C(c4ccc(N)cc4)C(C)C)cc3)cc2)cc1</smiles>
$87,(40 \%)$<smiles>O=C(CBr)c1ccc([N+](=O)[O-])cc1</smiles>

45<smiles>Nc1ccc(C(=O)Nc2cccc(Cl)c2)cc1</smiles>

31
$\mathrm{KHCO}_{3}$<smiles>[R]C=O</smiles>

(Figure 11) and others having 3-amino-N-phenylbenzamide as their core structure (Figure 12).

This research primarily focused on exploring the effect of a single substitution on either side of the anilinoketone/ ester. The rationale for this was to be able to study the effect of each substitution on its own, and then in a following study try to combine substitutions on both sides for more effective having 4-amino-N-phenylbenzamide as the core structure<smiles>O=C(CCl)Oc1ccccc1</smiles>

85<smiles>O=C(CCl)Oc1ccc2ccccc2c1</smiles>

86<smiles>Nc1ccc(C(=O)Nc2ccccc2)cc1</smiles>

23<smiles>O=C(CNc1ccc(C(=O)Nc2ccccc2)cc1)Oc1ccccc1</smiles>

$89(47 \%)$<smiles>Nc1ccc(C(=O)Nc2ccccc2)cc1</smiles><smiles>O=C(CNc1ccc(C(=O)Nc2ccccc2)cc1)Oc1ccc2ccccc2c1</smiles>

$90(44 \%)$

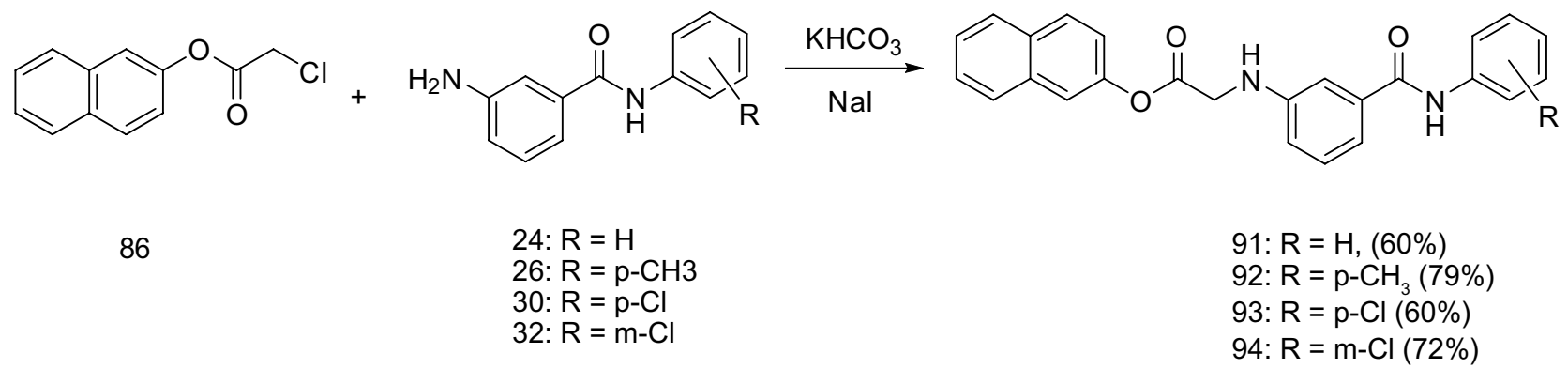

Figure 16 Synthesis of anilinoesters. 
inhibitors, and to get an idea of the effect of disubstitution on activity, two compounds with substitutions on both sides were proposed.

The yield of the anilinoketone synthesis varied over a wide range $(21 \%-89 \%)$. In general, compounds with meta substitutions had lower yields than those with para substitutions, probably as a result of the steric effect exerted by the meta substitution, which might have hindered the nucleophilic attack on the carbonyl. There was less variation in the anilinoester synthesis yields $(44 \%-79 \%)$.

In the mass spectra of the anilinoketones and anilinoesters, the molecular ion peak was the base peak. A common fragment of the anilinoketones is one that results from elimination of the terminal aniline, giving a peak at $\mathrm{m} / \mathrm{z}=238$ or its equivalent in substituted compounds (Figure 17), while in the case of anilinoesters, an important fragment appears at 225 or its equivalent in substituted compounds, which results from beta cleavage of the amine (Figure 18).

\section{Reduction of anilinoketones}

Reduction of anilinoketones to anilinoalcohol was brought about using sodium borohydride as the reducing agent. ${ }^{22}$ The reaction was carried out in methanol with stirring at room temperature for 1-2 hours. The workup consisted of washing with water, with $1 \mathrm{M} \mathrm{HCl}$, and then with water again. The organic layer was dried and evaporated, and the product was recrystallized from ethyl acetate/hexane.

The reactions and yields of the anilinoketone reductions are shown in Figure 19. The reaction yields ranged from $40 \%$ to $82 \%$, with the only exception being the reduction of compound 82 to the corresponding alcohol, ie, compound 113 , which had a $26 \%$ yield. One possible explanation for the low yield of this particular reaction is that the presence of the ortho substitution hindered formation of a complex between sodium borohydride and the oxygen on the ketone.

The variations in chemical yield can be attributed to many factors, ranging from human skill to the nature of the chemicals involved. For example, because these compounds were mainly purified by recrystallization, the skill of the worker can play a major role in choosing the best crystallization solvent. On the other hand, the nature of the substituents in the compound has a major role in the crystallization yield, which may differ according to whether the group is electronwithdrawing or electron-donating, and the size and position of these groups are also important. For compound 82, the ortho methoxy group sterically hinders formation of the intermediate complex between sodium borohydride and the ketone group, so the yields will be less than for other positions of the methoxy group.

The infrared spectra of the produced alcohols confirmed the reduction. In the spectra of the anilinoketones, there were usually two peaks in the carbonyl stretching region, ie, one for the ketone and another for the amide, but in the infrared spectra for anilinoalcohol, the ketone carbonyl peak disappeared and an alcohol peak appeared at around 3,450-3,300 $\mathrm{cm}^{-1}$.

The infrared spectrum for compound 57 (the most active compound) is shown in Figure 20. This spectrum shows the $\mathrm{N}-\mathrm{H}$ stretch at $3,365 \mathrm{~cm}^{-1}$ and $3,282 \mathrm{~cm}^{-1}$; the peak seen at $3,059 \mathrm{~cm}^{-1}$ represents the $\mathrm{C}-\mathrm{H}$ stretch; the carbonyl stretch is shown at $1,691 \mathrm{~cm}^{-1}$ and $1,543 \mathrm{~cm}^{-1}$; and finally, the C-N stretch appeared at $1,332 \mathrm{~cm}^{-1}$. The infrared spectra for compound 90 and other representative compounds are provided in the Supplementary materials section (Figures S1-S5).

The reaction was also confirmed by the NMR spectra for the products. Two extra peaks were observed in the spectra of anilinoalcohols when compared with those of the anilinoketones, ie, a peak at around 5.5 representing the proton of the $\mathrm{OH}$ group, and a peak at around 4.8 representing the $\mathrm{CH}$ proton that is attached to the $\mathrm{OH}$.

The $\mathrm{CH}_{2}$ bound to the aniline nitrogen appeared in the NMR spectra of the anilinoketones as a doublet at around 4.5 , but in the spectra of the anilinoalcohols, the two hydrogens became nonequivalent and appeared as two multiplets at around 3.2 and 3.3. This is usually observed in rigid systems such as small rings. A possible explanation would be that an intramolecular hydrogen bond forms between the $\mathrm{OH}$ and the neighboring $\mathrm{NH}$, resulting in a five-membered ring. In the ${ }^{13} \mathrm{C}-\mathrm{NMR}$ spectra of the anilinoketones, the
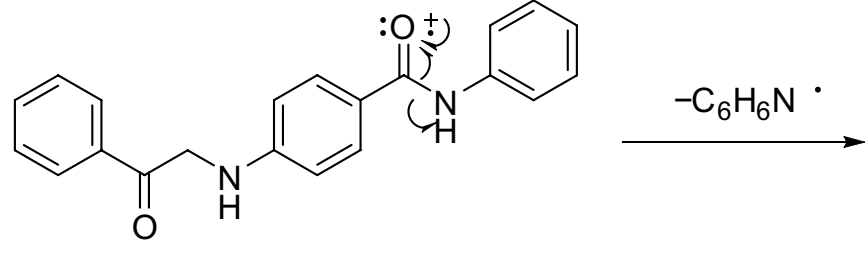

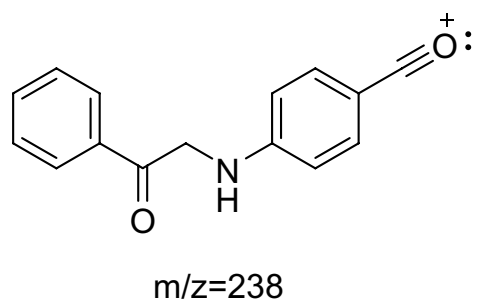

Figure 17 Mass fragmentation of anilinoketones. 

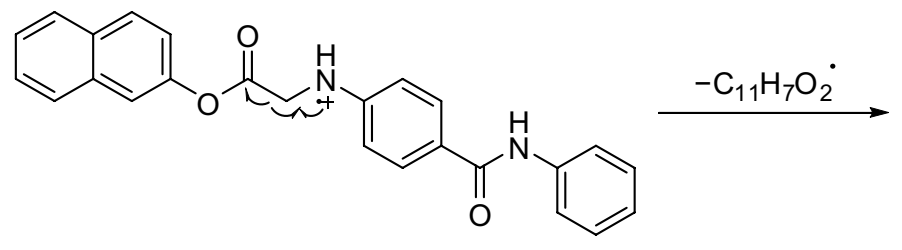

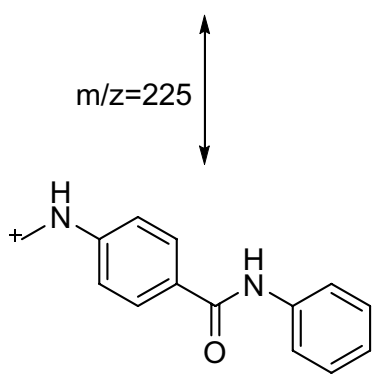

Figure 18 Mass fragmentation of anilinoesters.

carbonyl carbon appeared in the range of 190-200, but in the anilinoalcohols, this peak disappeared and the carbon attached to the $\mathrm{OH}$ appeared in the range of 65-71. The same type of mass fragmentation of the amide group was observed here as in the anilinoketones, and resulted in a peak at 240 or its equivalent in substituted compounds (Figure 21A). Another common fragmentation in anilinoalcohols was the loss of the alcohol group, resulting in a peak at 314 or its equivalent (Figure 21B).

The DPP-IV drug discovery kit from Enzo Life Sciences was used to determine the biological activity of the synthesized compounds. The kit contains a chromogenic substrate (H-Gly-Pro-pNA), and when the enzyme interacts with the substrate, the result is cleavage of p-nitroaniline from the substrate, with production of the p-nitroaniline elevating the absorption to $405 \mathrm{~nm}$.

All the synthesized compounds were screened at a concentration of $100 \mu \mathrm{M}$. The components of the kit were added in the order and amount indicated in the kit manual. The plate was incubated for 10 minutes and read every $2-3$ minutes over a 20 -minute period.

The slope of the absorbance plotted against time was calculated for each compound, and percent inhibition was calculated using the following formula:

Percent inhibition $=(1-$ slope of the compound $/$ slope of the negative inhibitor) $* 100 \%$

The plot of the negative control shows how the activity of the enzyme should be without the interference of any inhibitors, and the positive control shows the case of optimum inhibition. The activity of any compound is inferred from its ability to reduce the slope compared with that of the negative control.
As can be seen in Figure 22, the positive inhibitor reduced the slope to almost zero. On the other hand, compound 57, used here as representative of the tested compounds, had an effect almost in the middle of that of both controls, and almost all tested compounds had a similar effect.

The negative control contains the enzyme with the natural substrate, which is active, with no enzyme inhibitors to hinder it. Thus, the enzyme will be working to cleave the color-producing substrate, leading to increased absorbance with time. This negative inhibitor measures the activity of the enzyme under normal conditions. The positive control is found when the enzyme, the substrate, and the standard inhibitor provided by the kit are all together, and the inhibitor shows full inhibition of the enzyme, so there is no increase in absorbance because the substrate is not cleaved. The curve of our inhibitor is located between the negative inhibitor and the positive control curves because it is not active at the level of the positive control and at the same time has some activity to stop the enzyme partially. The percent inhibition values for the synthesized compounds are reported in Tables 2-7.

Our attempt to determine the $\mathrm{IC}_{50}$ of the top scoring compounds failed because of their low solubility in the solvent system used for testing, ie, assay buffer (Tris, pH 7.5) and DMSO in the ratio of $98.5: 1.5 \mathrm{v} / \mathrm{v}$. The procedure consisted of preparing four solutions of the compounds to be tested at concentrations of $0.1 \mu \mathrm{M}, 1 \mu \mathrm{M}, 10 \mu \mathrm{M}$, and $100 \mu \mathrm{M}$. While the compounds were soluble at $0.1 \mu \mathrm{M}$ and $1 \mu \mathrm{M}$, a visible precipitate was formed at $10 \mu \mathrm{M}$ and $100 \mu \mathrm{M}$. This is to be expected given that the compounds used in this research were lipophilic with calculated $\log \mathrm{P}$ values ranging from 3.13 to 5.83 .

The entire assay was performed at a concentration of $100 \mu \mathrm{m}$ for screening purposes. At this concentration, 
<smiles>[R]c1cccc(NC(=O)c2ccc(NCC(=O)c3ccccc3)cc2)c1</smiles>

52: $\mathrm{R}=\mathrm{H}$

54: $\mathrm{R}=\mathrm{p}-\mathrm{CH}_{3}$

58: $\mathrm{R}=\mathrm{p}-\mathrm{Cl}$

60: $\mathrm{R}=\mathrm{m}-\mathrm{Cl}$

62: $\mathrm{R}=\mathrm{p}-\mathrm{OMe}$

64: $\mathrm{R}=\mathrm{m}-\mathrm{OMe}$

68: $\mathrm{R}=\mathrm{m}-\mathrm{COCH}$

70: $\mathrm{R}=\mathrm{p}-\mathrm{COOCH}_{2} \mathrm{CH}_{3}$<smiles>[R]c1cccc(NC(=O)c2cccc(NCC(=O)c3ccccc3)c2)c1</smiles>

53: $\mathrm{R}=\mathrm{H}$

55: $\mathrm{R}=\mathrm{p}-\mathrm{CH}_{3}$

57: $\mathrm{R}=\mathrm{m}-\mathrm{CH}_{3}$

59: $\mathrm{R}=\mathrm{p}-\mathrm{Cl}$

61: $\mathrm{R}=\mathrm{m}-\mathrm{Cl}$

63: $\mathrm{R}=\mathrm{p}-\mathrm{OMe}$

65: $\mathrm{R}=\mathrm{m}-\mathrm{OMe}$

67: $\mathrm{R}=\mathrm{p}-\mathrm{COCH}_{3}$<smiles>[R][Z]1cccc(C(=O)CNc2ccc(C(=O)Nc3ccccc3)cc2)c1</smiles>

79: $\mathrm{R}=\mathrm{m}-\mathrm{OCH}_{3}$

80: $\mathrm{R}=\mathrm{p}-\mathrm{OCH}_{3}$

82: $\mathrm{R}=\mathrm{O}-\mathrm{OCH}_{3}^{3}$

83: $\mathrm{R}=\mathrm{p}-\mathrm{CF}_{3}$

84: $\mathrm{R}=\mathrm{p}-\mathrm{F}$

85: $\mathrm{R}=\mathrm{m}-\mathrm{NO}_{2}$

86: $\mathrm{R}=\mathrm{p}-\mathrm{Br}$

88: $\mathrm{R}=\mathrm{m}-\mathrm{Cl}$

89: $\mathrm{R}=\mathrm{p}-\mathrm{CH}_{3}$<smiles>O=C(CNC(=O)c1ccc(NC(=O)c2ccccc2)cc1)c1ccc2ccccc2c1</smiles>

90<smiles>[R]c1cccc(NC(=O)c2ccc(NCC(O)c3ccccc3)cc2)c1</smiles>

95: $\mathrm{R}=\mathrm{H},(48 \%)$

96: $\mathrm{R}=\mathrm{p}-\mathrm{CH}_{3},(70 \%)$

97: $\mathrm{R}=\mathrm{p}-\mathrm{Cl},(68 \%)$

98: $\mathrm{R}=\mathrm{m}-\mathrm{Cl},(40 \%)$

99: $\mathrm{R}=\mathrm{p}-\mathrm{OMe},(64 \%)$

100: $\mathrm{R}=\mathrm{m}-\mathrm{OMe},(56 \%)$

101: $\mathrm{R}=\mathrm{m}-\mathrm{COCH}_{3},(78 \%)$

102: $\mathrm{R}=\mathrm{p}-\mathrm{COOCH}_{2} \mathrm{CH}_{3},(58 \%)$

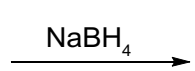<smiles>[R]CCCCNc1cccc(NC(=O)c2cccc(NCC(O)c3ccccc3)c2)c1</smiles>

103: $\mathrm{R}=\mathrm{H},(68 \%)$

104: $\mathrm{R}=\mathrm{p}-\mathrm{CH}_{3},(60 \%)$

105: $\mathrm{R}=\mathrm{m}-\mathrm{CH}_{3},(76 \%)$

106: $\mathrm{R}=\mathrm{p}-\mathrm{Cl},(74 \%)$

107: $\mathrm{R}=\mathrm{m}-\mathrm{Cl},(78 \%)$

108: $R=p-O M e, ~(70 \%)$

109: $\mathrm{R}=\mathrm{m}-\mathrm{OMe},(40 \%)$

110: $\mathrm{R}=\mathrm{p}-\mathrm{COCH}_{3},(59 \%)$

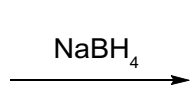<smiles>[R]c1cccc(C(O)CNc2ccc(C(=O)Nc3ccccc3)cc2)c1</smiles>

111: $\mathrm{R}=\mathrm{m}-\mathrm{OCH}_{3},(74 \%)$

112: $\mathrm{R}=\mathrm{p}-\mathrm{OCH}_{3},(65 \%)$

113: $\mathrm{R}=\mathrm{O}-\mathrm{OCH}_{3},(26 \%)$

114: $\mathrm{R}=\mathrm{p}-\mathrm{CF}_{3},(56 \%)$

115: $R=p-F,(82 \%)$

116: $\mathrm{R}=\mathrm{m}-\mathrm{NO}_{2},(76 \%)$

117: $\mathrm{R}=\mathrm{p}-\mathrm{Br},(70 \%)$

118: $\mathrm{R}=\mathrm{m}-\mathrm{Cl},(82 \%)$

119: $\mathrm{R}=\mathrm{p}-\mathrm{CH}_{3},(66 \%)$

$\mathrm{NaBH}_{4}$<smiles>O=C(Nc1ccccc1)c1ccc(NCC(O)c2ccc3ccccc3c2)cc1</smiles>

$120,(78 \%)$

Figure 19 Synthesis of substituted 4-(2-hydroxy-2-phenyl-ethylamino)-N-phenyl-benzamide, synthesis of substituted 3-(2-hydroxy-2-phenyl-ethylamino)-N-phenylbenzamide, synthesis of substituted 4-(2-hydroxy-2-phenyl-ethylamino)-N-phenyl-benzamide, and synthesis of naphthalen-2-yl (4-phenylcarbamoyl-phenylamino)-acetate.

some samples showed clear precipitate formation, so the actual amount that is soluble is very hard to determine given that there is no precise way to measure the amount of precipitate formed. However, the trend was that lipophilic compounds suffered from a salting-out effect, and if this happens, most probably they are not available for inhibition of the enzyme, which would be another problem in performing the $\mathrm{IC}_{50}$.

The fact that the compounds investigated in this project were insoluble at high concentrations raises the question of whether they also had low solubility at $100 \mu \mathrm{M}$ (the concentration at which the percent inhibition was calculated), 


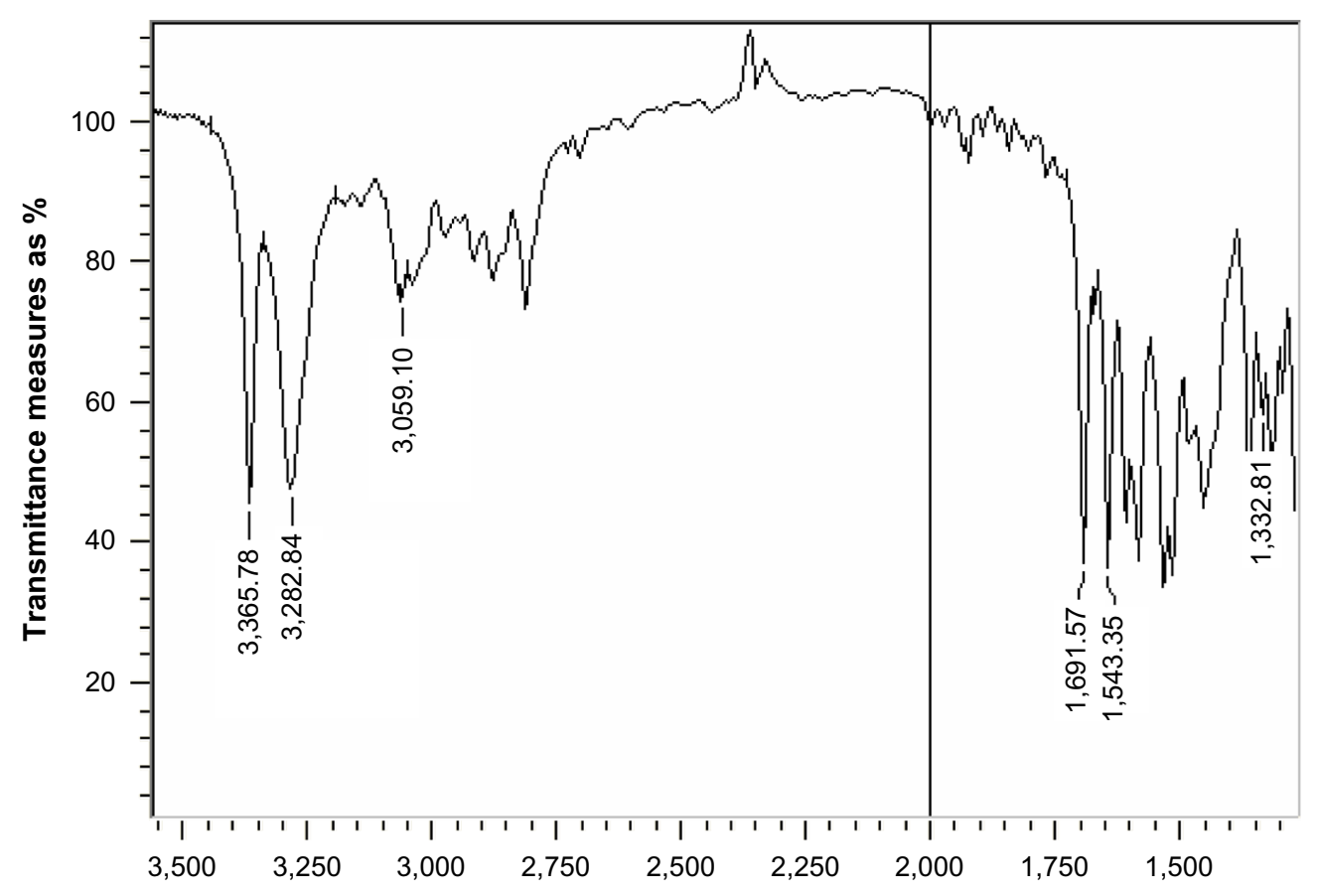

Wavenumber measures $1 / \mathrm{cm}$

Figure 20 Infrared spectrum of 57, the most active compound.

that went undetected due to the very low amount of the compound in the solution. This could account for the low activity of some of the compounds. The concentration of DPP-IV inhibitors used in the current study was higher than the concentrations normally achieved by these inhibitors. This is because our study represents an initial investigation of the tested compounds, and future studies will be carried out to optimize the activity of these molecules after resolving issues related to polarity and increasing their target affinity. Additionally, the in vitro drug concentration-activity relationship does not necessarily reflect the in vivo drug profile, given that drugs usually undergo absorption and metabolism steps that alter the final plasma drug concentration. There are no reported data concerning any inhibitor possess the same chemical scaffold related to our compounds that what make our compounds new lead, so it is not directly possible<smiles>O=C(Nc1ccccc1)c1ccc(NC(O)c2ccccc2)cc1</smiles>

A

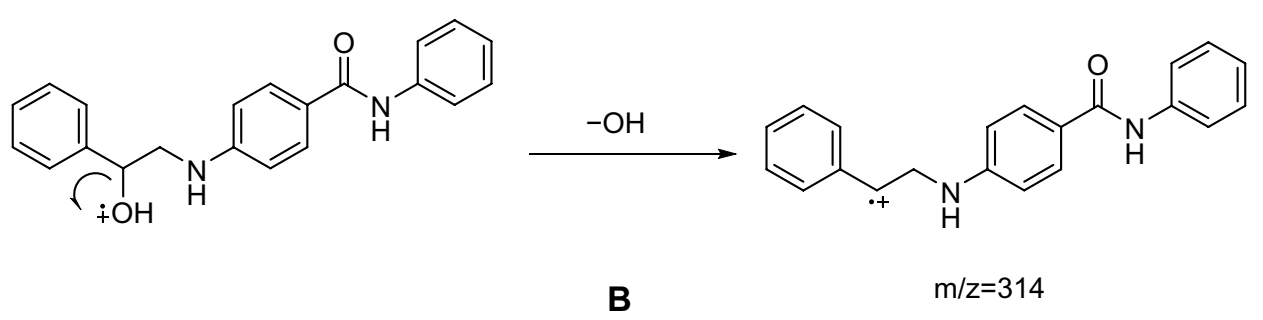

Figure 21 Mass fragmentation of anilinoalcohols. (A) Amide fragmentation and (B) $\mathrm{OH}$ loss. Note: Biological testing using DPP-IV assay kit. 


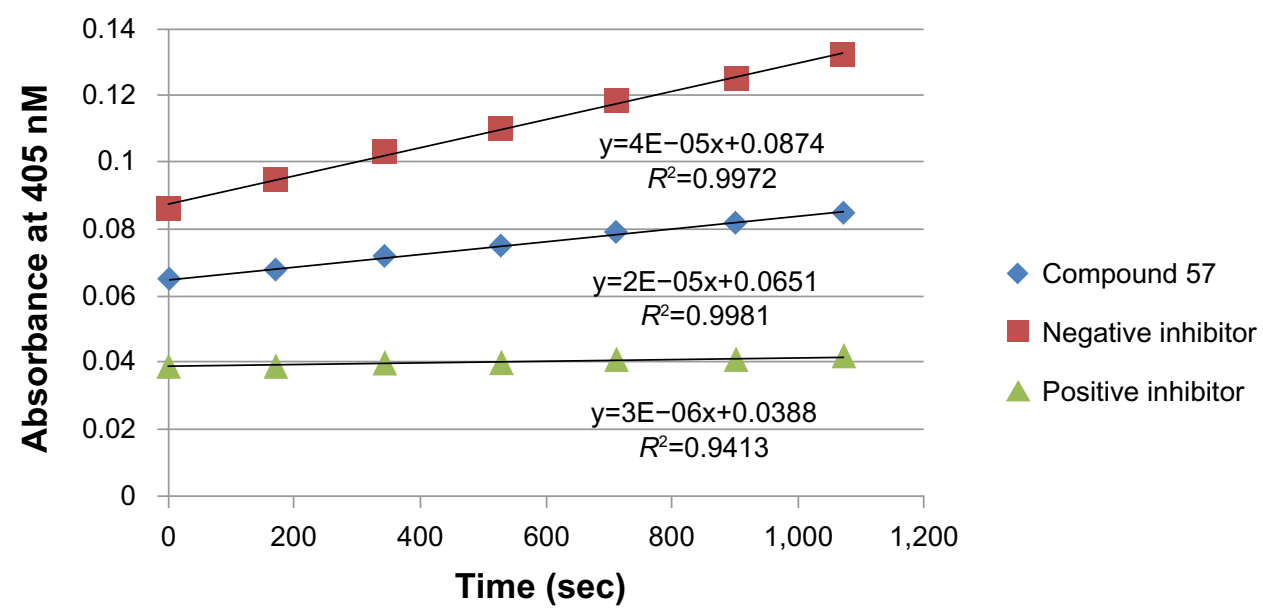

Figure 22 Plot of absorbance values versus time for compound 57, and the negative inhibitor and the positive inhibitor.

to correlate our compounds with any drug already on the market as they have around 0.10 micromolar concentrations in the plasma.

By examining the test results for the biological activity of the synthesized compounds, it can be seen that compounds 57 and 90 are the most active, with percent inhibition of $38 \%$

Table 2 Biological activity of anilinoketones with varying substitutions on ring I

\begin{tabular}{|c|c|c|c|}
\hline Compound & $\begin{array}{l}\text { Ring II } \\
\text { (meta or para) }\end{array}$ & $\mathbf{R}$ & $\begin{array}{l}\text { Percent } \\
\text { inhibition }\end{array}$ \\
\hline 52 & $\mathrm{P}$ & $\mathrm{H}$ & $4 \%$ \\
\hline 53 & $\mathrm{~m}$ & $\mathrm{H}$ & $32 \%$ \\
\hline 54 & $\mathrm{P}$ & $\mathrm{p}-\mathrm{CH}_{3}$ & $12 \%$ \\
\hline 55 & $\mathrm{~m}$ & $\mathrm{p}-\mathrm{CH}_{3}$ & $7 \%$ \\
\hline 56 & $\mathrm{P}$ & $\mathrm{m}-\mathrm{CH}_{3}$ & $14 \%$ \\
\hline 57 & $\mathrm{~m}$ & $\mathrm{~m}-\mathrm{CH}_{3}$ & $38 \%$ \\
\hline 58 & $\mathrm{P}$ & $\mathrm{p}-\mathrm{Cl}^{3}$ & $2 \%$ \\
\hline 59 & $\mathrm{~m}$ & $\mathrm{p}-\mathrm{Cl}$ & $24 \%$ \\
\hline 60 & $P$ & $\mathrm{~m}-\mathrm{Cl}$ & $0 \%$ \\
\hline 61 & $\mathrm{~m}$ & $\mathrm{~m}-\mathrm{Cl}$ & $0 \%$ \\
\hline 62 & $\mathrm{P}$ & $\mathrm{p}-\mathrm{OMe}$ & $0 \%$ \\
\hline 63 & $\mathrm{~m}$ & $\mathrm{p}-\mathrm{OMe}$ & $2 \%$ \\
\hline 64 & $\mathrm{P}$ & $\mathrm{m}-\mathrm{OMe}$ & $14 \%$ \\
\hline 65 & $\mathrm{~m}$ & $\mathrm{~m}-\mathrm{OMe}$ & $9 \%$ \\
\hline 66 & $P$ & $\mathrm{p}-\mathrm{COCH}_{3}$ & $23 \%$ \\
\hline 67 & $\mathrm{~m}$ & $\mathrm{p}-\mathrm{COCH}_{3}$ & $6 \%$ \\
\hline 68 & $P$ & $\mathrm{~m}-\mathrm{COCH}_{3}$ & $18 \%$ \\
\hline 69 & $\mathrm{~m}$ & $\mathrm{~m}-\mathrm{COCH}_{3}$ & $34 \%$ \\
\hline 70 & $\mathrm{P}$ & $\mathrm{p}-\mathrm{COOCH}_{2} \mathrm{CH}_{3}$ & $9 \%$ \\
\hline 71 & $\mathrm{~m}$ & $\mathrm{p}-\mathrm{COOCH}_{2} \mathrm{CH}_{3}$ & $30 \%$ \\
\hline 72 & $\mathrm{P}$ & $\mathrm{m}-\mathrm{COOCH}_{2} \mathrm{CH}_{3}^{3}$ & $1 \%$ \\
\hline 73 & $\mathrm{~m}$ & $\mathrm{~m}-\mathrm{COOCH}_{2} \mathrm{CH}_{3}$ & $18 \%$ \\
\hline
\end{tabular}

and $37 \%$, respectively. Twelve of the synthesized compounds had a percent inhibition $\geq 28 \%$ (note here that compound 1 , the lead compound for this research, had a percent inhibition of $28 \%$ ).

Comparing the biological activity of alcohols and ketones, the compounds with varying substitutions on ring I (Table 6), most of the alcohols were more active than their corresponding ketones. One possible explanation is that the ability of an alcohol to act as a hydrogen bond donor gives it the ability to form an extra H-bond. Another possible explanation is that the relatively higher flexibility in the alcohols allows them to have better fitting in the active site. Three exceptions appear in Table 6, ie, compounds 96, 99, and 101, which had

Table 3 Biological activity of anilinoketones with varying substitutions on ring III

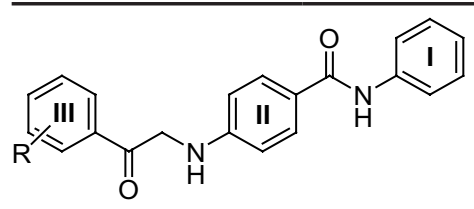

\begin{tabular}{lll} 
Compound & $\mathbf{R}$ & Percent inhibition \\
\hline 74 & $\mathrm{p}-\mathrm{NO}_{2}$ & $19 \%$ \\
75 & $\mathrm{~m}-\mathrm{OCH}_{3}$ & $0 \%$ \\
76 & $\mathrm{p}-\mathrm{OCH}_{3}$ & $28 \%$ \\
77 & $\mathrm{p}-\mathrm{CN}$ & $21 \%$ \\
78 & $\mathrm{O}-\mathrm{OCH}_{3}$ & $0 \%$ \\
79 & $\mathrm{p}-\mathrm{CF}_{3}$ & $22 \%$ \\
80 & $\mathrm{p}-\mathrm{F}$ & $22 \%$ \\
$8 \mathrm{I}$ & $8 \%$ \\
82 & $\mathrm{~m}-\mathrm{NO}_{2}$ & $33 \%$ \\
83 & $\mathrm{p}-\mathrm{Br}$ & $1 \%$ \\
84 & $\mathrm{~m}-\mathrm{Br}$ & $19 \%$ \\
85 & $\mathrm{~m}-\mathrm{Cl}$ & $0 \%$ \\
86 & $\mathrm{p}-\mathrm{CH}_{3}$ & $25 \%$ \\
& $(\mathrm{To}$ form & \\
\hline
\end{tabular}


Table 4 Biological activity of disubstituted anilinoketones

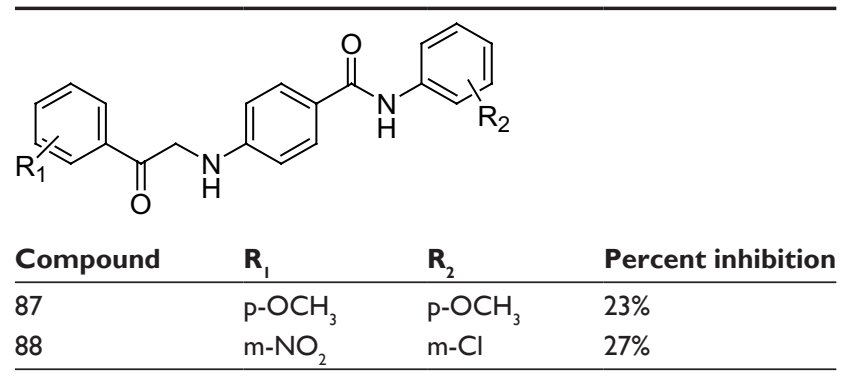

significantly lower activity than their corresponding ketones. The three compounds have a meta-substituted ring II, which could have placed the ketone in a more preferable position to act as a hydrogen bond acceptor.

The trend was less obvious in compounds with varying substitutions on ring III (Table 7). Half of these compounds followed the trend, but the other half showed very similar percent inhibition when comparing the ketones with their corresponding alcohols. This suggests that having substitutions on ring III may have changed the mode of binding to a position that was neutral for the ketone and alcohol groups.

Comparing meta-substitution versus para-substitution on ring II in ketones with varying substitutions on ring I, compounds with a meta-substituted ring II were generally more active than those with a para-substituted ring. However, the trend was reversed in alcohols of this group, with the para-substituted one being the more active of the two. This makes sense if we assume that both the ketone and the alcohol groups form an H-bond with the same amino acid. In this case the ketone needs to be closer since the oxygen will be forming the bond (hence the meta is better); on the other hand, the alcohol is probably forming the bond

Table 5 Biological activity of anilinoesters

\begin{tabular}{|c|c|c|c|c|}
\hline Compound & Ring II & $\mathbf{x}$ & $\mathbf{R}$ & Percent inhibition \\
\hline 89 & Para & Phenyl & $\mathrm{H}$ & $6 \%$ \\
\hline 90 & Para & Naphthyl & $\mathrm{H}$ & $37 \%$ \\
\hline 91 & Meta & Naphthyl & $\mathrm{H}$ & $1 \%$ \\
\hline 92 & Meta & Naphthyl & $\mathrm{p}-\mathrm{CH}_{3}$ & $22 \%$ \\
\hline 93 & Meta & Naphthyl & $\mathrm{p}-\mathrm{Cl}$ & $0 \%$ \\
\hline 94 & Meta & Naphthyl & $\mathrm{m}-\mathrm{Cl}$ & $15 \%$ \\
\hline
\end{tabular}

through the hydrogen, so needs some space (hence the para is better).

For substitution of meta versus para in peripheral substitutions, there was no obvious trend in substitutions in ketones with varying substitutions on ring I, which makes each compound a unique case. In ketones with varying substitutions on ring III, the ortho and meta substitutions were generally less active than the para substitutions, which may be due to the steric effect exerted by these substitutions. Again, however, this trend was lost in the alcohols.

In general, compounds with substitutions capable of forming $\mathrm{H}$-bonds were not significantly more active than those with hydrophobic substitutions. This might suggest that in the majority of cases these compounds failed to form the expected H-bond. Some compounds with hydrophobic substitutions such as $57\left(\mathrm{~m}-\mathrm{CH}_{3}\right), 82(\mathrm{p}-\mathrm{Br})$, and $103(\mathrm{~m}-\mathrm{Cl})$, were active with percent inhibition of $38 \%, 33 \%$, and $23 \%$, respectively. In these cases, it seems that the hydrophobic groups were suitably placed in a hydrophobic region of the active site and resulted in high activity.

Some compounds with groups forming H-bonds were also active, including 69 (m-acetyl), 106 (m-methoxy), 111 (m-methoxy), 116 (m-nitro), and 113 (o-methoxy), with percent inhibition values of $34 \%, 32 \%, 31 \%, 30 \%$, and $29 \%$ respectively. These compounds seem to have been favorably placed in the active site in a pose that allowed the H-bond to form.

For anilinoesters, compound 90 was active with a percent inhibition of $37 \%$. The naphthyl group could have formed a highly effective $\pi-\pi$ interaction with the active site, resulting in this high activity. There is a possibility that this high activity was a result of the extra oxygen forming an H-bond, but since compounds 89 and 91, which have the oxygen in a similar position, were almost inactive, this might indicate that the oxygen was not involved in the binding. The other anilinoesters were not as active, probably because the meta-substituted ring II did not place the naphthyl group in the optimum position to form the $\pi-\pi$ interaction.

The two compounds with disubstitutions both had good activity, suggesting that an increase in the size of the compound is permissible provided that the added groups are chosen carefully. One of the compounds had two para substitutions while the other had two meta substitutions, meaning that both types of substitutions are acceptable. Compound 87, which has two methoxy substitutions, had almost the same percent inhibition as compound 76, which only has one methoxy, meaning that despite the second 
Table 6 Biological activity of anilinoalcohols with varying substitutions on ring I<smiles>[R]c1ccc(NC(=O)c2ccc(NCC(O)c3ccccc3)cc2)cc1</smiles>

\begin{tabular}{|c|c|c|c|c|}
\hline Compound & $\begin{array}{l}\text { Ring II } \\
\text { (meta or para) }\end{array}$ & $\mathbf{R}$ & $\begin{array}{l}\text { Percent inhibition } \\
\text { of anilinoalcohol }\end{array}$ & $\begin{array}{l}\text { Percent inhibition of } \\
\text { corresponding ketone }\end{array}$ \\
\hline 95 & $\mathrm{P}$ & $\mathrm{H}$ & $33 \%$ & $4 \%$ \\
\hline 96 & $\mathrm{~m}$ & $\mathrm{H}$ & $4 \%$ & $32 \%$ \\
\hline 97 & $\mathrm{P}$ & $\mathrm{p}-\mathrm{CH}_{3}$ & $15 \%$ & $12 \%$ \\
\hline 98 & $\mathrm{~m}$ & $\mathrm{p}-\mathrm{CH}_{3}$ & $8 \%$ & $7 \%$ \\
\hline 99 & $\mathrm{~m}$ & $\mathrm{~m}-\mathrm{CH}_{3}$ & $3 \%$ & $38 \%$ \\
\hline 100 & $\mathrm{P}$ & $\mathrm{p}-\mathrm{Cl}$ & $27 \%$ & $2 \%$ \\
\hline 101 & $\mathrm{~m}$ & $\mathrm{p}-\mathrm{Cl}$ & $0 \%$ & $24 \%$ \\
\hline 102 & $\mathrm{P}$ & $\mathrm{m}-\mathrm{Cl}$ & $20 \%$ & $0 \%$ \\
\hline 103 & $\mathrm{~m}$ & $\mathrm{~m}-\mathrm{Cl}$ & $23 \%$ & $0 \%$ \\
\hline 104 & $\mathrm{P}$ & $\mathrm{p}-\mathrm{OMe}$ & $26 \%$ & $0 \%$ \\
\hline 105 & $\mathrm{~m}$ & $\mathrm{p}-\mathrm{OMe}$ & $14 \%$ & $2 \%$ \\
\hline 106 & $\mathrm{P}$ & m-OMe & $32 \%$ & $14 \%$ \\
\hline 107 & $\mathrm{~m}$ & $\mathrm{~m}-\mathrm{OMe}$ & $24 \%$ & $9 \%$ \\
\hline 108 & $\mathrm{~m}$ & $\mathrm{p}-\mathrm{COCH}_{3}$ & $19 \%$ & $6 \%$ \\
\hline 109 & $\mathrm{P}$ & $\mathrm{m}-\mathrm{COCH}_{3}$ & $22 \%$ & $18 \%$ \\
\hline 110 & $\mathrm{p}$ & $\mathrm{p}-\mathrm{COOCH}_{2} \mathrm{CH}_{3}$ & $22 \%$ & $9 \%$ \\
\hline
\end{tabular}

substitution being tolerated, it did not increase the activity of the compound. Compound $88\left(\mathrm{~m}-\mathrm{Cl}\right.$ and $\left.\mathrm{m}-\mathrm{NO}_{2}\right)$ had significantly higher activity than compounds $60(\mathrm{~m}-\mathrm{Cl})$ and $81\left(\mathrm{~m}-\mathrm{NO}_{2}\right)$.

Table 7 Biological activity of anilinoalcohols with varying substitutions on cycle III<smiles>[R][X]c1ccccc1C(O)CNc1ccc(C(=O)Nc2ccccc2)cc1</smiles>

\begin{tabular}{|c|c|c|c|}
\hline Compound & $\mathbf{R}$ & $\begin{array}{l}\text { Percent } \\
\text { inhibition of } \\
\text { anilinoalcohol }\end{array}$ & $\begin{array}{l}\text { Percent inhibition } \\
\text { of corresponding } \\
\text { ketone }\end{array}$ \\
\hline 111 & $\mathrm{~m}-\mathrm{OCH}_{3}$ & $31 \%$ & $0 \%$ \\
\hline 112 & $\mathrm{p}-\mathrm{OCH}_{3}{ }_{3}$ & $20 \%$ & $28 \%$ \\
\hline 113 & $\mathrm{O}-\mathrm{OCH}_{3}$ & $29 \%$ & $0 \%$ \\
\hline 114 & $\mathrm{p}-\mathrm{CF}_{3}$ & $27 \%$ & $22 \%$ \\
\hline 115 & $\mathrm{p}-\mathrm{F}$ & $20 \%$ & $22 \%$ \\
\hline 116 & $\mathrm{~m}-\mathrm{NO}_{2}$ & $30 \%$ & $8 \%$ \\
\hline 117 & $\mathrm{p}-\mathrm{Br}$ & $18 \%$ & $33 \%$ \\
\hline 118 & $\mathrm{~m}-\mathrm{Cl}$ & $13 \%$ & $19 \%$ \\
\hline 119 & $\mathrm{p}-\mathrm{CH}_{3}$ & $23 \%$ & $0 \%$ \\
\hline 120 & 2-naphthyl & $25 \%$ & $25 \%$ \\
\hline
\end{tabular}

\section{Conclusion}

In this project, 69 novel compounds carrying the $\mathrm{N}$-substituted aminobenzamide scaffold were designed using advanced and validated molecular modeling software, synthesized for the first time, and tested for their ability to inhibit the DPP-IV enzyme, an essential antidiabetic target. These compounds showed a range of percent inhibition at $100 \mu \mathrm{M}$, with ten compounds scoring inhibition activity above that of the reference compound 1 . The highest compound scored $38 \%$ inhibition of activity of the enzyme, which is determined using validated and ready kits to ensure that there were no experimental errors during the assay. All 69 compounds were purified and fully characterized using proton and carbon NMR, infrared spectroscopy, and melting point determination. Full details of their identification are described in the Materials and methods section of this paper.

\section{Acknowledgment}

This work was supported by the Scientific Research Fund, Ministry of Higher Education and Scientific Research, The Hashemite Kingdom of Jordan (grant TS/3/14/2008) and the Scientific Research Deanship, Jordan University of Science and Technology (grant 11/2012). 


\section{Disclosure}

The authors report no conflicts of interest in this work.

\section{References}

1. Kuzuyaa T, Nakagawab S, Satohe J, et al. Committee of the Japan Diabetes Society on the diagnostic criteria of diabetes mellitus. Report of the committee on the classification and diagnostic criteria of diabetes mellitus. Diabetes Res Clin Pract. 2002;55:65-85.

2. Clark N, Fox K, Grandy S. Symptoms of diabetes and their association with the risk and presence of diabetes. Diabetes Care. 2007;30: 2868-2873.

3. Zimmet P. The burden of type 2 diabetes: are we doing enough? Diabetes Metab. 2003;29:6S9-6S18.

4. Ismail-Beigi F. Glycemic management of type 2 diabetes mellitus. N Engl J Med. 2012;366:1319-1327.

5. Hopsu-Havu V, Glenner G. [A new dipeptide naphthylamidase hydrolyzing glycyl-prolyl-beta-naphthylamide]. Histochemie. 1966;7:197-201. German.

6. Holst J, Deacon C. Perspectives in diabetes. Inhibition of the activity of dipeptidyl-peptidase IV as a treatment for type 2 diabetes. Diabetes Metab. 1998;47:1663-1670.

7. US Food and Drug Administration. Drugs@FDA. Available from: http://www.accessdata.fda.gov/scripts/cder/drugsatfda/index.cfm. Accessed April 13, 2013.

8. Lambeir A, Diaz Pereira J, Chacon P, et al. A prediction of DPP IV/CD26 domain structure from a physico-chemical investigation of dipeptidyl peptidase IV (CD26) from human seminal plasma. Biochim Biophys Acta. 1997;1340:215-226.

9. Lambeir A, Durinx C, Scharpé S, De Meester I. Dipeptidyl-peptidase IV from bench to bedside: an update on structural properties, functions, and clinical aspects of the enzyme DPP IV. Crit Rev Clin Lab Sci. 2003;40:209-294.

10. Ogata S, Misumi Y, Tsuji E, Takami N, Oda K, Ikehara Y. Identification of the active site residues in dipeptidyl peptidase IV by affinity labeling and site-directed mutagenesis. Biochemistry. 1992;31:2582-2587.
11. David F, Bernard A, Pierres M, Arguet D. Identification of serine 624, aspartic acid 702, and histidine 734 as the catalytic triad residues of mouse dipeptidyl-peptidase IV (CD26). J Biol Chem. 1993;268: $17247-17252$.

12. Abbott C, McCaughan G, Gorrell M. Two highly conserved glutamic acid residues in the predicted $\mathrm{L}$ propeller domain of dipeptidyl peptidase IV are required for its enzyme activity. FEBS Lett. 1999;458: 278-284.

13. Rasmussen H, Branner S, Wiberg F, Wagtmann N. Crystal structure of human dipeptidyl peptidase IV/CD26 in complex with a substrate analog. Nat Struct Biol. 2003;10:19-25.

14. Protein Data Bank. Biological macromolecular resource. Available from: http://www.rcsb.org/pdb/home/home.do. Accessed November 12, 2011.

15. Discovery Studio Modeling Suite. Version release 3.5. San Diego, CA, USA: Accelrys Software; 2013.

16. ChemDraw. Chemical drawing software (computer program). Version release 12.0. Cambridge, MA: PerkinElmer software; 2012.

17. White E. Deamination of amines. 2-phenylethyl benzoate via the nitrosoamide decomposition. Org Syn Coll. 1973;5:336-339.

18. Hodgson H, Hathway D. The preparation of some halogenonaphthylamines, dihalogenonaphthalenes, 2-bromo-1-naphthol, and related derivatives. J Chem Soc. 1944:538-539.

19. Beugelmans R, Singh G, Bois-Choussy M, Chastanet J, Zhu J. SNArbased macrocyclization: an application to the synthesis of vancomycin family models. J Org Chem. 1994;59:5535-5542.

20. Wang L, Li P, Wu Z, Yan J, Wang M, Ding Y. Reduction of nitroarenes to aromatic amines with nanosized activated metallic iron powder in water. Synthesis. 2003;13:2001-2004.

21. Loeser E, Prasad K, Repic O. Selective N-alkylation of primary amines with chloroacetamides under $\mathrm{pH}$-controlled aqueous conditions. Synth Commun. 2002;32:403-409.

22. Dilling W, Plepys R. Metal hydride reductions of endo-tricyclo[5.2.1.02,6] deca-4,8-dien-3-one-(endo -dicyclopentadienone). J Org Chem. 1970;35:2971-2976. 


\section{Supplementary materials}

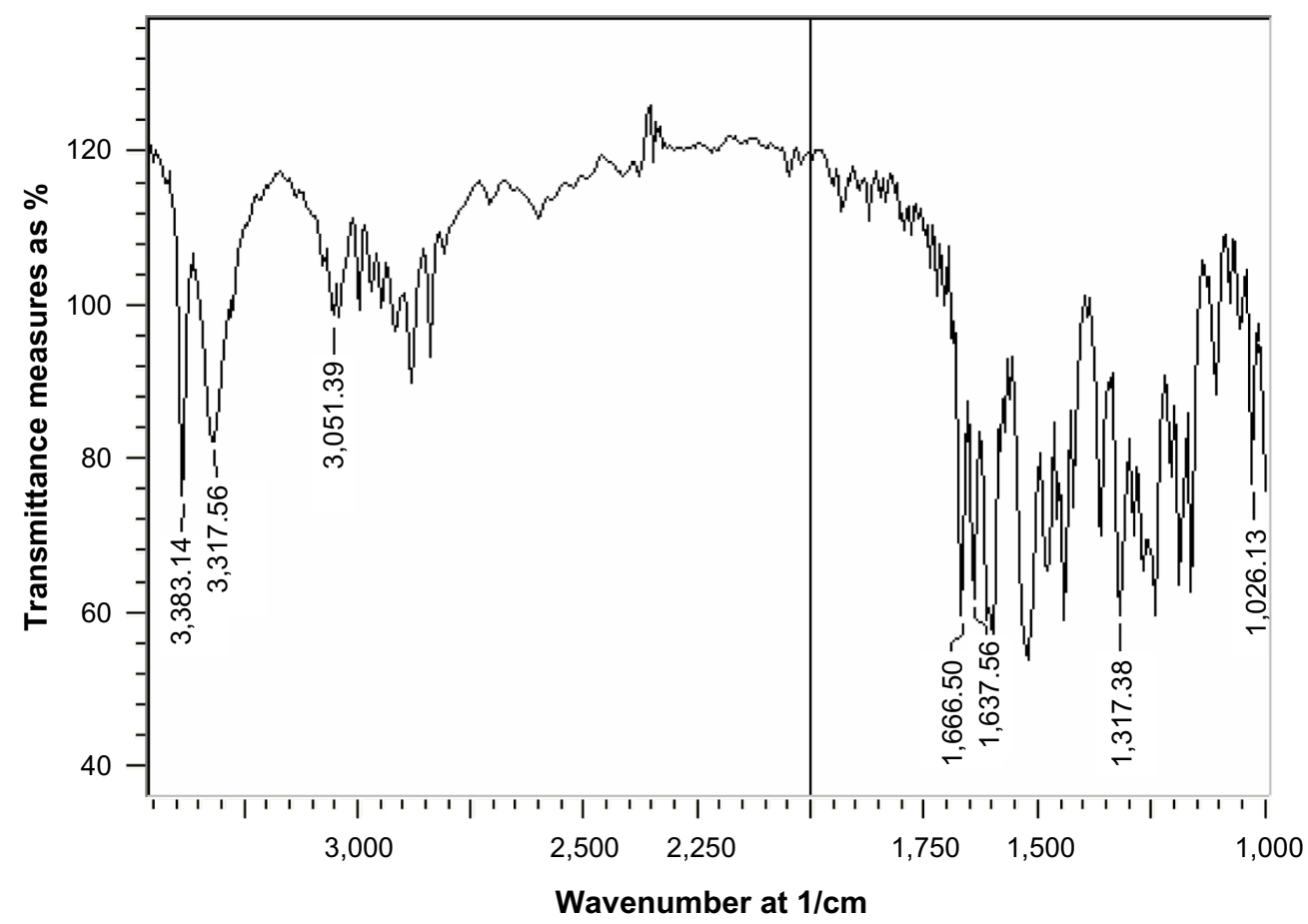

Figure SI Infrared spectrum of compound 78.

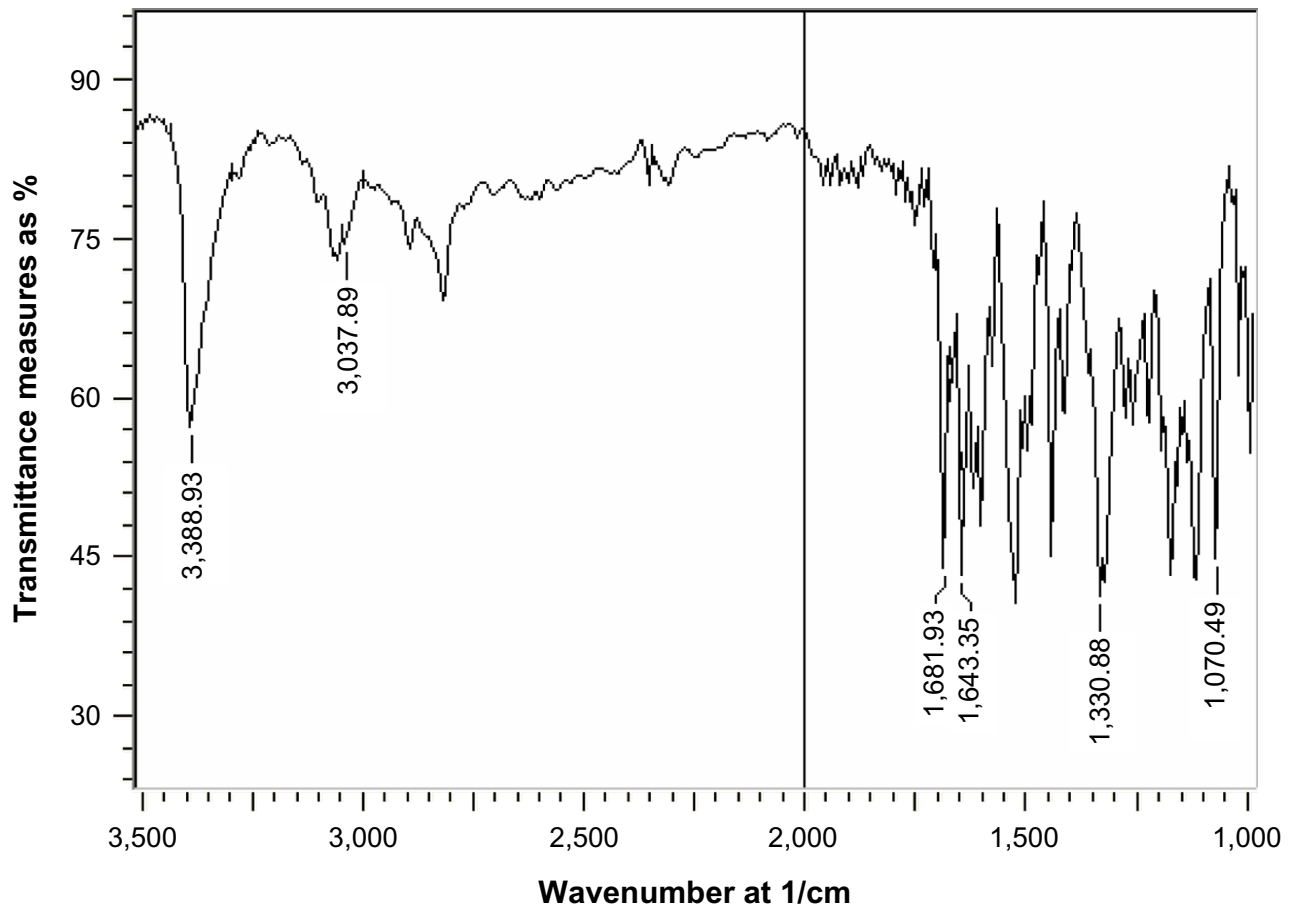

Figure S2 Infrared spectrum of compound 83. 


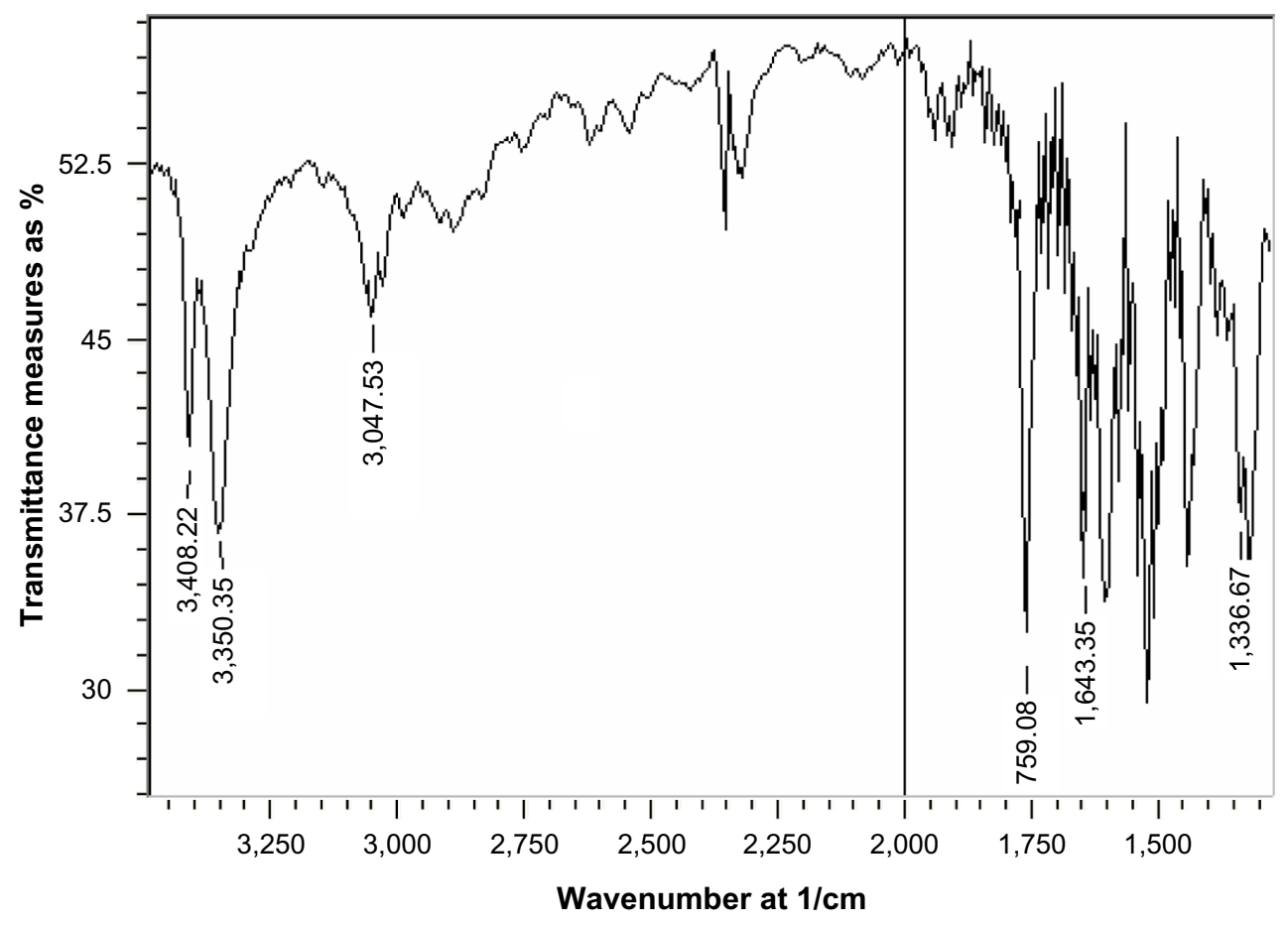

Figure S3 Infrared spectrum of compound 90.

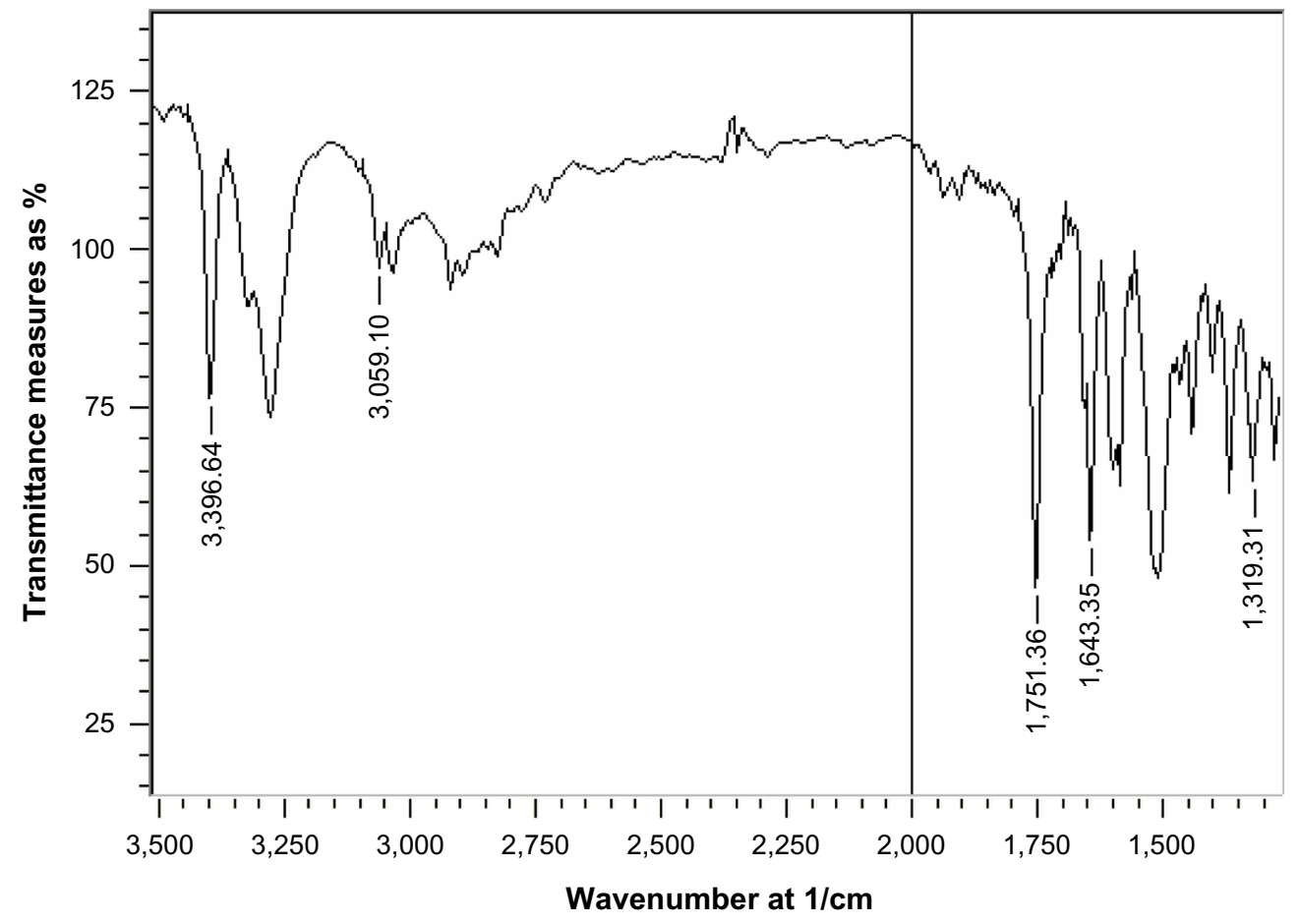

Figure S4 Infrared spectrum of compound 93. 


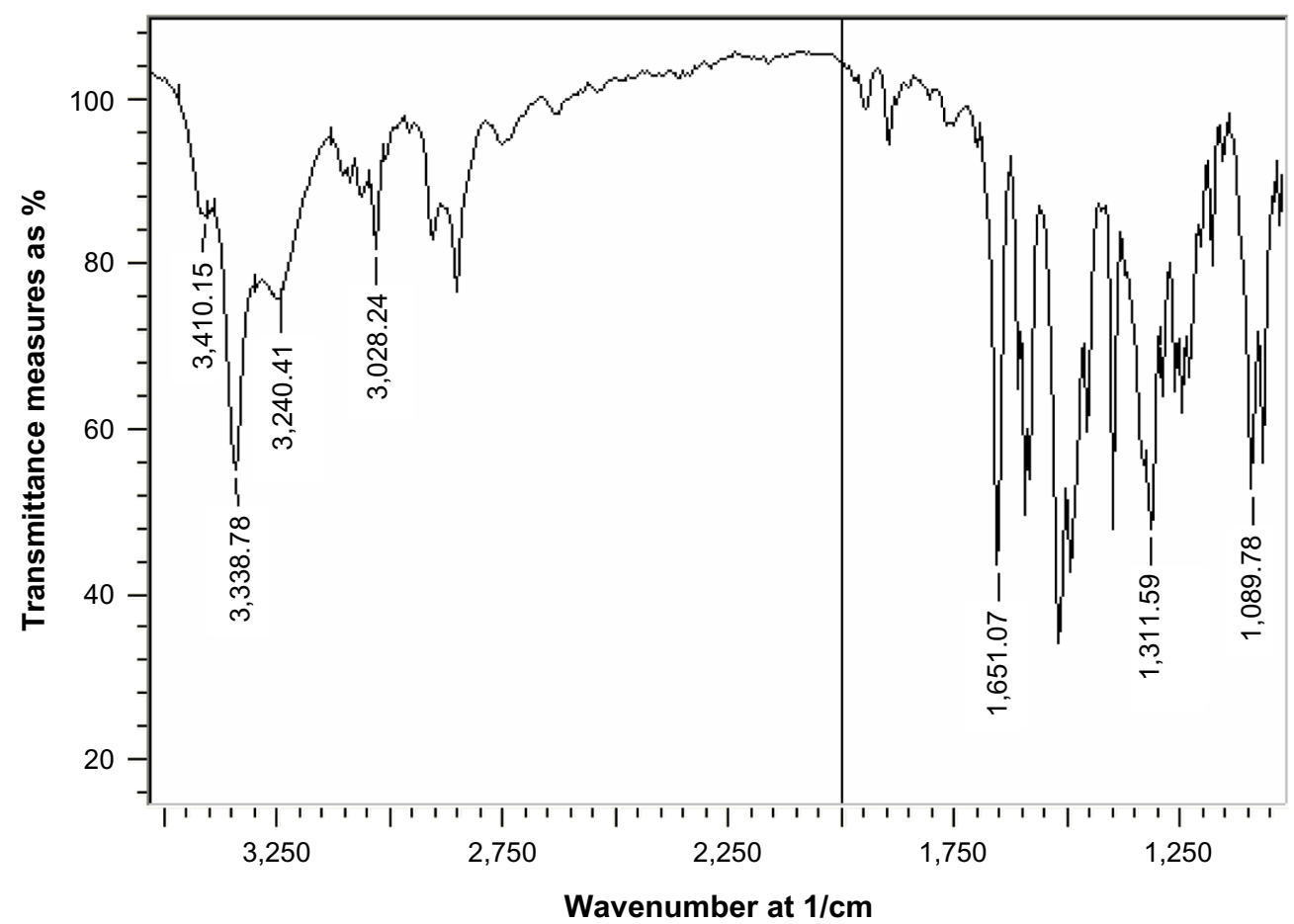

Figure S5 Infrared spectrum of compound 98.

\section{Publish your work in this journal}

Drug Design, Development and Therapy is an international, peerreviewed open-access journal that spans the spectrum of drug design and development through to clinical applications. Clinical outcomes, patient safety, and programs for the development and effective, safe, and sustained use of medicines are a feature of the journal, which has also been accepted for indexing on PubMed Central. The manuscript management system is completely online and includes a very quick and fair peer-review system, which is all easy to use. Visit http://www.dovepress.com/testimonials.php to read real quotes from published authors.

Submit your manuscript here: http://www.dovepress.com/drug-design-development-and-therapy-journal 